\title{
THREE ESSAYS IN THE ECONOMICS OF GROWTH WITH NATURAL RESOURCES AND ENVIRONMENT
}

\author{
by \\ Youssef Boudribila
}

\author{
A thesis submitted to the \\ Faculty of Graduate and Postdoctoral Affairs \\ in partial fulfillment of the requirements for the degree of \\ Doctor of Philosophy \\ Department of Economics \\ Faculty of Public Affairs \\ Carleton University \\ Ottawa, Ontario \\ Canada
}

October 2012

Copyright (C) Youssef Boudribila 2012 
Library and Archives

Canada

Published Heritage

Branch

395 Wellington Street

Ottawa ON K1A ON4

Canada
Bibliothèque et

Archives Canada

Direction du

Patrimoine de l'édition

395 , rue Wellington

Ottawa ON K1A ON4

Canada
Your file Votre référence

ISBN: 978-0-494-94210-9

Our file Notre référence

ISBN: $978-0-494-94210-9$
NOTICE:

The author has granted a nonexclusive license allowing Library and Archives Canada to reproduce, publish, archive, preserve, conserve, communicate to the public by telecommunication or on the Internet, loan, distrbute and sell theses worldwide, for commercial or noncommercial purposes, in microform, paper, electronic and/or any other formats.

The author retains copyright ownership and moral rights in this thesis. Neither the thesis nor substantial extracts from it may be printed or otherwise reproduced without the author's permission.
AVIS:

L'auteur a accordé une licence non exclusive permettant à la Bibliothèque et Archives Canada de reproduire, publier, archiver, sauvegarder, conserver, transmettre au public par télécommunication ou par l'Internet, prêter, distribuer et vendre des thèses partout dans le monde, à des fins commerciales ou autres, sur support microforme, papier, électronique et/ou autres formats.

L'auteur conserve la propriété du droit d'auteur et des droits moraux qui protege cette thèse. $\mathrm{Ni}$ la thèse ni des extraits substantiels de celle-ci ne doivent être imprimés ou autrement reproduits sans son autorisation.
In compliance with the Canadian Privacy Act some supporting forms may have been removed from this thesis.

While these forms may be included in the document page count, their removal does not represent any loss of content from the thesis.
Conformément à la loi canadienne sur la protection de la vie privée, quelques formulaires secondaires ont été enlevés de cette thèse.

Bien que ces formulaires aient inclus dans la pagination, il n'y aura aucun contenu manquant. 



\begin{abstract}
This thesis is composed of three essays. Essay one of the thesis presents an attempt in the recent efforts to find the closed form solution of the HamiltonJacobi-Bellman equation for models with homogeneous preferences and constantreturns-to scale technologies. The method is illustrated by finding the closedform Bellman function for the AK model of endogenous growth and the model of economic growth with exhaustible resources. The closed-form Bellman functions thus obtained are exploited to analyze the time paths of consumption, capital accumulation, and resource depletion.

Essay two extends the famous model of Dasgupta and Heal to explore the question raised in 1972, about the limits to growth. It presents an endogenous growth model with an exhaustible resource, say fossil fuels, with a perfect substitute, which is produced by a backstop. The capital stock is allocated between the consumption good sector and the backstop. It is shown that the resource is exploited first, and is depleted in finite time. The backstop takes over the function of meeting the energy needs of the economy immediately after the resource is exhausted: a transition phase during which the two technologies are simultaneously exploited does not exist. After the resource stock has been exhausted, the economy enters immediately into a steady state and grows along a balanced growth path of sustainable development.

Essay three extend the previous model to the world of climate change, to make a link between growth and the increasing risk of a catastrophic cli-
\end{abstract}


mate change. In the long run, and under the scenario that no catastrophe occurs, the economy converges to a balanced growth path. If the initial volume of greenhouse gases in the atmosphere is high, consumption will follow an U-shape pattern. On the other hand, if the rate of time preferences and the parameter that characterizes the low probability of catastrophe are low, consumption will be rising through time. The current Hamiltonian is used to compute Green GDP, which takes into consideration the costs of resource depletion, the degradation of the environment caused by the emissions of GHG, and the loss due to a possible catastrophic climate change. 


\section{ACKNOWLEDGMENTS}

First and foremost, I humbly thank to God as it is His blessing, in all aspects, that I have been able to accomplish my dissertation.

The writing of my thesis has been a strenuous journey, which I could not have accomplished without the passionate and continued support of advisors, colleagues, friends and family.

I will remain profoundly grateful to my thesis supervisor Professor Nguyen Van Quyen, for carefully reviewing all my work. He has very kindly given numerous ideas and precious suggestions and guidance. His ideas and suggestions have been so valuable and convincing that helped me to the completion of this dissertation. His guidance helped me to understand how to correctly make the link between maths and economics, and how to develop a broader structural vision when making this link. I am proud to be his Ph.D. student.

I am deeply grateful to my external examiner; Professor Hassan Benchekroun, to dissertation committee; Professor Ba Chu, Professor Jankiang Zhang, and Professor Jean Thomas Bernard for their time, their detailed reviews and excellent advices for refining my thesis. I also appreciate for their critical comments, which enabled me to notice the invaluable points to clarify my research questions and to make the necessary improvements according to their comments.

I want to express my sincere thanks to Professor Linda Khalaf for all the support she gave me all along this process. I will remain profoundly grateful to 
her for her involvement and encouragement to make this work accomplished. Throughout of my graduate study, her encouragement and help made me feel confident to overcome every difficulty I encountered.

I also want to thank Marge Brooks, our department administrator, for her kindness, help and high level of professionalism.

Special thanks to Mohsen Bouaissa for his valuable support and motivations during the last period of this process.

I thank to my parents, as they are not with us, but their wishes and prayers are always with me. I also thank to my brother, sisters for their great encouragement and support. 
To my Father, the silent teacher,...

to my Mother, the school of patience...

to my Sisters, the resistant fighters...

to my brothers, the dreamers. 


\section{Contents}

Table of Contents $\quad$ viii

List of Figures $\quad$ xi

$\begin{array}{ll}\text { Introduction } & 1\end{array}$

1 The Closed-Form Bellman Function for Optimal Economic Growth Models with Homogeneous Preferences and Constant Returns to Scale Technology $\mathbf{8}$

1.1 Introduction . . . . . . . . . . . . . . . . . 8

1.2 The AK model ....................... 11

1.2.1 Formal statement of the problem . . . . . . . . . . 11

1.2 .2 The optimal-value function . . . . . . . . . . . . 12

1.2.3 The Hamilton-Jacobi-Bellman equation for the AK model . . 14

1.2.4 The current Hamiltonian . . . . . . . . . . . . . . . 16

1.2.5 Maximization of the current Hamiltonian . . . . . . . . . 17

1.2.6 The closed-form solution of the Hamilton-Jacobi-Bellman equation for the AK model . . . . . . . . . . . . . . 18

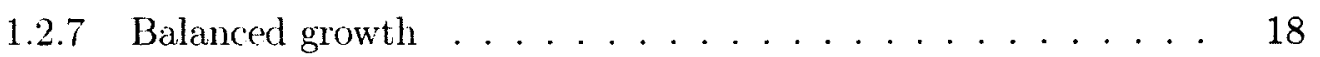

1.3 Economic growth with exhaustible resources . . . . . . . . . . 20

1.3.1 Formal statement of the problem . . . . . . . . . 20

1.3.2 The optimal-value function . . . . . . . . . . . . 21

1.3.3 The optimal-value function when the economy has capital but no fossil fuels .................. . . . 24

1.3.4 The optimal-value function when the economy has both capital and fossil fuels . . . . . . . . . . . . . 26

1.3.5 The time paths of resource depletion, capital accumulation, and

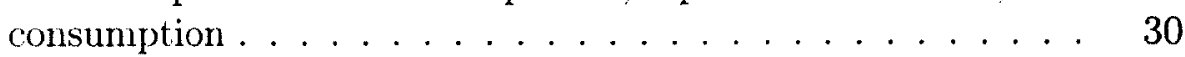

1.4 Concluding remarks . . . . . . . . . . . . . 33

Appendix 1.A Derivation of the HJB equation and the adjoint equations . $\quad 37$

Appendix 1.B The derivation of the Bellman function . . . . . . . . . 38 


\section{Exhaustible Resources and Technology Substitution in a Model of} Endogenous Growth

2.1 Introduction . . . . . . . . . . . . . . . . . . 49

2.2 The model . . . . . . . . . . . . . . . . . . . . . . . . 53

2.2 .1 Preferences . . . . . . . . . . . . . . . . . . 53

2.2 .2 The energy technologies . . . . . . . . . . . . . . 53

2.2 .3 The consumption good technology . . . . . . . . . . . 54

2.2.4 Formal statement of the optimal growth problem with two energy sectors . . . . . . . . . . . . . 55

2.3 The Hanilton-Jacobi-Bellman equation . . . . . . . . . . 56

2.4 The post-fossil fuel phase . . . . . . . . . . . . . 60

2.5 The fossil fuel phase . . . . . . . . . . . . . 63

2.6 A characterization of the optimal solution $\ldots \ldots \ldots \ldots \ldots 74$

2.7 A numerical example . . . . . . . . . . . . . . . . . 74

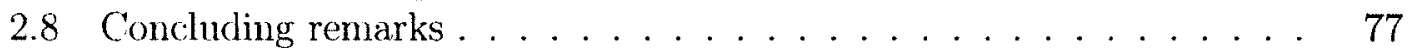

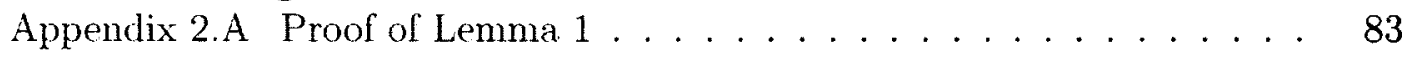

Appendix 2.B The derivation of the HJB equation and the adjoint equations 85 Appendix 2.C The closed-form expression for the capital stock before oil exhaustion . . . . . . . . . . . . . . . . 9 91

\section{The Low-Probability High-Consequence Events In Environmental} Economics

3.1 Introduction $\ldots \ldots \ldots \ldots \ldots \ldots \ldots \ldots \ldots$

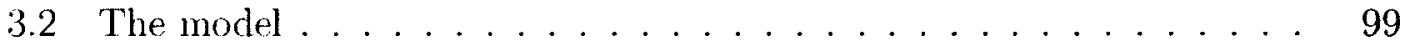

3.2 .1 Preferences . . . . . . . . . . . . . . . . . 100

3.2 .2 The energy technologies . . . . . . . . . . . 100

3.2 .3 The consumption good technology . . . . . . . . . . 100

3.2.4 Catastrophe, its probabilities and consequences . . . . . . 101

3.2.5 Formal statement of the optimal growth problem with two energy sectors and possible climate change . . . . . . . . 103

3.3 The Hamilton-Jacobi-Bellman Equation $\ldots \ldots \ldots \ldots . \ldots . \ldots$

3.4 Maximization of the Hamiltonian . . . . . . . . . . . . 107

3.4.1 The motion of the shadow prices . . . . . . . 108

3.4 .2 The first-order conditions . . . . . . . . . . . . 110

3.5 Green national income . . . . . . . . . . . . . . . . . . 112

3.6 A characterization of the optimal solution $\ldots \ldots \ldots \ldots \ldots$

3.7 Concluding remarks . . . . . . . . . . . . . . . . . . 124

Appendix 3.A The derivation of the HJB equation and the adjoint equations131

3.A.1 The Hamilton-Jacobi-Bellman equation . . . . . . . . . . 131

3.A.2 The motion of the shadow prices . . . . . . . . 133

3.A.2.1 The motion of the shadow price of oil . . . . . . 135

3.A.2.2 The motion of the shadow price of capital . . . . 137 
3.A.2.3 The motion of the shadow price of greenhouse gases in the atmosphere . . . . . . . . . . 138 


\section{List of Figures}

2.1 Time path of the extraction rate of fossil fuel $\ldots \ldots \ldots 75$

2.2 Time path of physical capital accumulation . . . . . . . 76

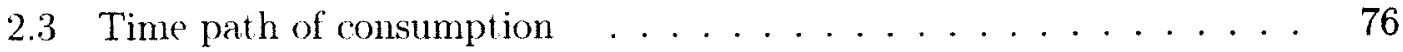




\section{Introduction}

This dissertation presents three essays on economic growth in production economies using energy derived from exhaustible natural resources. The first essay is a methodological contribution to the issue of finding the closed form solution of the value function in the class of homogeneous dynamic optimization problems, which encompasses most of economic growth with exhaustible resources models. For this class of models, among several interesting results, a general solution method for finding the closed form solution for the Hamilton-Jacobi-Bellman (HJB) equation (which typically characterizes the system dynamics along the optimal path) when optimal value function is homogeneous is presented. The second essay applies these results to study a dynamic general equilibrium model of depletion of exhaustible resources in an economy where renewable energy input is produced by a backstop technology. The third essay further introduces catastrophic events in this economy, occurring randomly after a climate change has resulted from environmental problems caused by the use of the exhaustible resources (e.g. the accumulation of greenhouse gases in the atmosphere due to the burning of fossil fuels).

The maximum principle (Pontryagin et al. 1962) is the standard tool for solving continuous time models of economic growth. The solution to the agent(s) optimization problem in these models is obtained by choosing at each instant the controls that maximize the Hamiltonian. As the optimal controls obtained with this approach 
are functions of the state and co-state variables, the analysis of the dynamics of these variables is required to fully solve the problem. These dynamics are governed by complex differential equations for which closed form solutions cannot generally be obtained, except for some special, and rather simple, cases. Without a closed form solution, the computation of the optimal path, the transition to the long-run equilibrium, and the impact of the initial conditions on the optimal path are not possible. Most often, researchers overcome this difficulty by using phase diagrams to study the qualitative behaviour of the economy in its convergence to the steady state. An alternative approach to the maximum principle, that avoids many difficulties and technical complications, is to exploit the dynamic programming framework to derive the HJB equation characterizing the optimal solution of a continuous time dynamic optimization problem. This approach has several advantages. First, the derivation of the HJB equation requires the continuity of the partial derivatives of the the optimal value function, a requirement typically satisfied by economic models. Second, the optimal value function, which satisfies the HJB equation, has economic contents, and its partial derivatives provide the shadow prices of the state variables. Third, with dynamic programming, the complex, and and often intractable, partial differential equation form of the HJB equation can be transformed into a system of ordinary differential equations, much easier to solve, so that the Pontryagin's maximum is obtained with considerably less effort.

In the first essay, I show how the closed-form solution of the HJB equation can be found by exploiting the homogeneity of the optimal value function. The homogeneity of the value function in dynamic programming problems obtains when the return function is homogeneous and the constraints are linearly homogeneous. The homogeneity property is preserved and transferred to the optimal value function. This provides valuable information about its functional form and helps finding its closed 
form solution, which in turn allows a direct and in-depth analysis of the optimal solution, in addition to revealing its economic contents. I illustrate the method by solving two well known economic growth models: the Rebelo (1991) AK model of endogenous growth, and Dasgupta and Heal (1974) model of economic growth with exhaustible resources. The latter is analysed in the specific case of equality between the capital share of output and the inverse of the inter-temporal elasticity of substitution. This is obviously a very restrictive assumption, but the approach I use can be seen as a first step towards finding the closed form for the Bellman function of more general versions of this model.

The second essay extends the model of Dasgupta and Heal (1974) to address the question about the limits to growth raised four decades ago. At that time, Meadows et. al (1972) published the infamous book "The Limits to Growth," which, based on the simulation of a computable model, predicts that the world economy will soon run out of key mineral resources and drown in pollution. Economists objected to these dire predictions by claiming that new substitutes will be found for the minerals in short supplies, and technological progress will solve both the scarcity and the environmental problems. The pessimistic conclusions of Meadows et. al (1972) and the high level of energy prices stimulated economists interest in investigating the limits imposed by exhaustible resources and the environment on economic growth, thus initiating a literature strand on growth sustainability ( see, for example, the symposium on the Economics of Exhaustible Resources organized by the Review of Economic Studies in 1974). In relation to this literature, I set up and study in Essay two an endogenous growth model, where production uses an exhaustible resource as input, for which a perfect substitute is produced by a backstop technology. Within this model, preferences exhibit constant inter-temporal elasticity of substitution, and the economy dynamics are linearly homogeneous in the control and state variables. I 
analyze the model using the findings of the first essay, and provide a detailed analysis of the economics of the derived welfare function. More specifically, I investigate the pattern of resource depletion, the problem of technology substitution, and their impacts on economic growth. I derive the HJB equation characterizing the optimal solution of the model, and analyze the balanced growth path of the economy after depletion of the fossil fuels stock, as well as the preceding phase where that stock is still exploited. The optimal solution is further illustrated through a numerical example.

Compared to other models on economic growth with exhaustible resource, the model I develop has the distinctive feature of allowing for technology substitution for the exhaustible resource. In the classic model of resource depletion of Dasgupta and Heal (1974), the economy collapses in the long run when the resource runs out. The literature on endogenous growth with exhaustible resources has shown that endogenous technological progress can counteract the negative effect of resource depletion and allow for a sustainable or rising level of consumption. Such a scenario is likely only when the possibility of technology substitution exists. This is precisely the case in my model, where the resource stock is exploited first, and the backstop technology is brought into use only after the exhaustion of the resource. The resource stock is also depleted in finite time, instead of being meagrely stretched out until the economy collapses in the long run, as in Dasgupta and Heal (1974). Furthermore, consumption no longer falls monotonically through time to zero, but depending on the parameters of the model, consumption might rise at first, reach a peak, and then decline, as in Pezzy and Withagen (1998) and Hartwick et al. (2003). This is because the technology substitution might induce the economy to accumulate capital more rapidly by curbing consumption at the beginning. I also show that, alternatively, if capital is scarce, but resources are abundant, consumption will fall initially, descends to its 
lower level, and then begins to ascend before resource exhaustion.

The use of exhaustible resources may cause serious environmental problems. The burning of fossil fuels, for example, generates greenhouse gases (GHGs), and might lead to climate change. The concern is about the emission of carbon dioxide $\left(\mathrm{CO}_{2}\right)$, methane $\left(\mathrm{CH}_{4}\right)$, nitrous oxide $\left(\mathrm{N}_{2} \mathrm{O}\right)$, water vapour $\left(\mathrm{H}_{2} \mathrm{O}\right)$, etc., the major greenhouse gases (GHGs) that are responsible for climate change. The International Panel of Climate Change (IPCC) concluded in 2001 that there is new and stronger evidence that most of the warming observed over at least the past fifty years is attributable to human activities (IPCC-2001). In a later report (IPCC-2007), the agency stated that continued GHG emissions at or above current rates would cause further warming and induce many changes in the global climate system during the 21 st century that would very likely be larger than those observed during the 20th century. In fact, the share of fossil fuels in current energy primary use amounts to $80 \%$ globally, and energy consumption will increase by $44 \%$ globally in 2030 (EIA 2006). The catastrophic consequences of climate change might dampen the enthusiasm for the fossil fuel technology, and lead to a more judicious exploitation of the resource. This issue is investigated in the third essay, where the model economy of the second essay is recast within a world of climate change.

The climate change may lead to catastrophic event that can be worse than disasters already experienced and recorded in the nearest past such as hurricanes, forest fires, drought, floods, species extinctions, irreversible damages to some ecosystems, etc. There is then a fairly serious risk of major irreversible change. To explore the economic impact of such events, I develop a stochastic model of economic growth where the burning of fossil fuels raises the stock of GHGs in the atmosphere, possibly leading to climate change that causes catastrophic events (e.g. a rise in the sea level, severe droughts, the melting of the permafrost that triggers a massive release of the 
GHGs trapped in). When it occurs, such an event causes massive damages to the stock of man-made and natural capitals, and raises the stock of GHGs in the atmosphere. The model assumes that a catastrophic event might occur only once, and when it does, the irreversible damage to the environment is such that consumption permanently jumps to its lowest level, normalized to zero for tractability purposes, as is customary in the related literature. The low probability of occurrence and arrival time of such a catastrophe are modelled through a Poisson process, with intensity depending on the stock of GHGs in the atmosphere.

In this model's economy, a consumption good is produced with labour, capital, and a composite energy input. The composite energy input is generated by burning fossil fuels, by exploiting a backstop technology, or by using both of them. Fossil fuels (i.e. oil) can be extracted at negligible cost, but their stock is limited. Energy produced from the backstop, on the other hand, requires capital (e.g. solar collectors, wind turbines, etc.) As oil resources dwindle, the backstop must be brought into use to prevent a drastic reduction in consumption. Also, in the context of climate change, this substitution for fossil fuels with renewable energy reduces GHGs emissions, which lowers the probability of catastrophic events. For these reasons, the substitution for energy generated by burning fossil fuels by renewable energy is a key feature of the model, besides being a critical economic issue. However, with the introduction of random jumps in the model, the homogeneity of the value function is lost, and finding the closed-form solution of the HJB equation becomes a very difficult task. As in the second essay, I use dynamic programming to derive the HJB equation for the stochastic growth problem of the economy described above. My derivations of the HJB equation and the differential equations governing the motion of the shadow prices of oil, capital, and GHGs, use the dynamic programming-based heuristic arguments of Bekovits (1974). Among other results, I show that along the optimal path, the 
value of the current Hamiltonian at each point in time represents the green GDP at that instant. 


\section{Essay 1}

\section{The Closed-Form Bellman}

\section{Function for Optimal Economic}

\section{Growth Models with Homogeneous}

\section{Preferences and Constant Returns}

\section{to Scale Technology}

\subsection{Introduction}

The standard tool for formalizing and then analyzing models of economic growth is the maximum principle developed by Pontryagin and his associates (1962). The solution is obtained by choosing at each instant the controls that maximize the Hamiltonian. The optimal controls thus obtained depend on the value of the state and co-state variables at that instant, and this requires the analysis of the motion of these variables. Except for very simple models, it is not possible to find the closed-form solutions of 
the differential equations that govern the motion of the state and co-state variables. Thus, researchers often resort to the use of phase diagrams to study the qualitative behavior of the system in its convergence to the steady state. Furthermore, without a closed-form solution, it is not possible to actually compute the optimal path, the transition to the long-run equilibrium, and the impact of the initial conditions on the optimal path.

Recently, various attempts have been made to find the closed-form solutions of some well known models of economic growth by assuming homogeneous preferences and Cobb-Douglas technology. See, for example, Smith (2006) and Guerrini (2010), who find the closed-form solution for the Ramsey growth model -for the case the utility function has constant inter-temporal elasticity of the substitution and the technology is of the Cobb-Douglas type- by finding the closed-form solutions for the differential equations that govern the behavior of the state and co-state variables. The important assumption made by these researchers is that the share of capital in national income is equal to the reciprocal of the intertemporal elasticity of substitution. The closed-form solutions for economic growth models with two factors of production - reproducible capital and an exhaustible resource of Dasgupta and Heal (1974), Solow (1974), and Stiglitz (1974) have also been found by Benchekroun and Withagen (2010). The closed-form solution for the system of differential equations that characterize the central planner's solution to the two-sector endogenous growth model with human capital of Lucas (1988) has been provided by Ruiz-Tamarit (2008).

The proof of the maximum principle given by Pontryagin et al. is long and hard. An alternative approach is to exploit the dynamic programming formalism and derive the Hamilton-Jacobi-Bellman equation, which characterizes the optimal solution of a dynamic optimization problem in continuous time. This approach has several advantages. First, the derivation of the Hamilton-Jacobi-Bellman equation requires 
the optimal-value function to have continuous partial derivatives, which does not present a problem in economics. Second, the optimal-value function, which satisfies the Hamilton-Jacobi-Bellman equation, has economic contents, and its partial derivatives can be interpreted as the shadow prices of the state variables. However, a major disadvantage of the Hamilton-Jacobi-Bellman equation is that it is a partial differential equation, and only rarely is it possible to obtain the closed-form solution of such a partial differential equation. Thus, it is more convenient to transform this partial differential equation, again by exploiting the dynamic programming formalism, into a system of ordinary differential equations, and in this manner the Pontryagin's maximum can be obtained with considerably less effort.

In some cases, the closed-form solution of the Hamilton-Jacobi-Bellman equation can be found, especially when the optimal-value function is homogeneous. It is well known in discrete dynamic programming that the Bellman function is homogeneous if the return function is homogeneous and the constraints are linear homogeneous; see, for example, Stokey (1996) and Alvarez and Stokey (1998). In the endogenous growth models of Lucas (1988), Jones and Manuelli (1990), and Rebelo (1991), the utility function used in these models has a constant inter-temporal elasticity of substitution, and the production function is linear homogeneous, and it can be shown that the structure of these models leads to the property that the optimal-value function is homogeneous of the same degree as the utility function.

In this paper, I show how the closed-form solution for the Hamilton-JacobiBellman can be found when the optimal-value function is homogeneous. I illustrate the solution technique by solving two well known models of economic growth: the AK model of Rebelo (1991) and the model of economic growth with an exhaustible resources of Dasgupta and Heal (1974) and that of Stiglitz (1974).

The paper is organized as follows. In Section 2, the AK model of Rebelo is 
presented, and its closed-form Bellman function is derived. The closed-form Bellman function is then used to analyze the solution of the model. The model of growth with exhaustible resources of Dasgupta and Heal is presented in Section 3. The closedform Bellman equation for the model is derived, and then used to analyze the dynamic behavior of the system. The derivation of the Hamilton-Jacobi-Bellman equation for this model is relegated to the appendix. Section 4 contains some concluding remarks.

\subsection{The AK model}

\subsubsection{Formal statement of the problem}

In the model, time is continuous and denoted by $t, t \geq 0$. The economy produces a consumption good, using labor and capital. The consumption good can also be used as investment goods to augment the stock of capital. In the AK model, capital is broadly defined so that it includes physical capital, human capital, and the stock of knowledge.

Let $u(c)$ denote the instantaneous utility of consumption, I assume that

$$
u(c)=\frac{c^{1-\theta}}{1-\theta}
$$

where $\theta, 0<\theta<1$, is a parameter, which is the inverse of the inter-temporal elasticity of substitution.

The consumption good is produced according to the following technology:

$$
Y=A F(K, L)
$$

where $Y$ represents output, $K$ the capital input, and $L$ the labor input. Also, $A$ is the technological level. The population is assumed to be constant and normalized to 1. In what follows, I assume that $F(K, 1)$ has the linear form:

$$
F(K, 1)=A K
$$


which implies that with a fixed labor input there is constant returns to scale with respect to the capital input. The production function represented by $(1.2 .3)$ is thus the technology of the $A K$ model of endogenous growth à la Arrow (1962) and Romer (1986). It is the linear structure of the production technology that drives growth.

I assume that capital does not depreciate. If we denote by $c_{t}$ the consumption at time $t$ and $K_{t}$ the capital stock, also at time $t$, then the motion of the stock of capital is governed by the following differential equation:

$$
\begin{aligned}
\frac{d K}{d t}= & A K_{t}-c_{t} \\
& K_{0} \text { is given. }
\end{aligned}
$$

By a consumption program, I mean a non-negative piece-wise and right-continuous function $t \rightarrow c_{t}, t \geq 0$. A consumption program is said to be feasible if the solution of $(1.2 .4)$ is such that $K_{t} \geq 0$ for all $t \geq 0$.

Let $\left(c_{t}\right)_{t \geq 0}$ be a feasible consumption program. The discounted value of the stream of social welfare associated with $\left(c_{t}\right)_{t \geq 0}$ is given by

$$
\int_{0}^{\infty} e^{-\rho t} u\left(c_{t}\right) d t
$$

In $(1.2 .5), \rho>0$ is the rate of time preferences.

The problem faced by the central planner is to find a feasible consumption program $\left(c_{t}\right)_{t>0}$ to maximize $(1.2 .5)$ subject to $(1.2 .4)$.

\subsubsection{The optimal-value function}

Suppose that the economy begins at time $\tau \geq 0$ with $\eta$ as the capital stock. Let

$$
v(\eta, \tau)=\max _{\left(c_{t}\right)_{t \geq \tau}} \int_{\tau}^{\infty} e^{-\rho t} u\left(c_{t}\right) d t
$$


subject to

$$
\begin{aligned}
\frac{d K}{d t} & =\Lambda K_{t}-c_{t} \\
K_{\tau} & =\eta
\end{aligned}
$$

As defined, $v(\eta, \tau)$ represents the optimal present value of social welfare, given that the economy begins at time $\tau$ with $\eta$ as the stock of capital. Note that when $\eta=K_{0}$ and $\tau=0$, the maximization problem constituted by (1.2.6) and (1.2.7) is reduced to the original problem, which is that of maximizing (1.2.5) subject to (1.2.4).

The optimal-value function $v(\eta, \tau)$ has the following obvious properties. First, $v(0, \tau)=0$, i.e., when there is no capital to begin with, there will be no production, and the discounted social welfare will be zero. The economy cannot exist under this scenario. Second, discounted social welfare is continuous and strictly increasing in the stock of capital. Moreover, since the return function is concave and the constraint is linear, the value function is concave and there is a unique global optimum, Caputo (2005).The following lemma presents some important and less obvious properties of $v(\eta, \tau)$ that will be exploited to solve the Hamilton-Jacobi-Bellman equation.

Lemma 1. The optimal-value function $v(\eta, \tau)$ has the following properties:

(i) For each $\tau$, the function $v(\eta, \tau): \eta \rightarrow v(\eta, \tau)$ is homogeneous of degree $(1-\theta)$ in $\eta$. That is, for any $\lambda>0$, we have $v(\lambda \eta, \tau)=\lambda^{1-\theta} v(\eta, \tau)$.

(ii) $(1-\theta) v(\eta, \tau)=\eta D_{1} v(\eta, \tau)$., where $D_{1} v(\eta, \tau)$, denotes the partial derivatives of $v(\eta, \tau)$ with respect to $\eta$.

Proof. Let $c_{t}, l \geq \tau$, be the optimal consumption from the initial condition $(\eta, \tau)$ and $K_{t}, \iota \geq \tau$, be the associated time path of the capital stock. Next, note that for any $\lambda>0, \lambda c_{t}, t \geq \tau$, is a feasible consumption program from the initial condition 
$(\lambda \eta, \tau)$ and $\lambda K_{t}, t \geq \tau$, is the associated time path of the capital stock from this initial condition. The discounted social welfare under $\lambda c_{t}, t \geq \tau$, is thus given by:

$$
\begin{aligned}
\int_{\tau}^{\infty} e^{-\rho t} u\left(\lambda c_{t}\right) d t & =\int_{\tau}^{\infty} e^{-\rho t} \frac{(\lambda c)^{1-\theta}}{1-\theta} d t \\
& =\lambda^{1-\theta} \int_{\tau}^{\infty} e^{-\rho t} \frac{c^{1-\theta}}{1-\theta} d t \\
& =\lambda^{1-\theta} v(\eta, \tau) \leq v(\lambda \eta, \tau) .
\end{aligned}
$$

The argument used to obtain (1.2.8) can be applied to assert that

$$
v(\eta, \tau)=v\left(\frac{1}{\lambda} \lambda \eta, \tau\right) \geq\left(\frac{1}{\lambda}\right)^{1-\theta} v(\lambda \eta, \tau)
$$

i.e.,

$$
\lambda^{1-\theta} v(\eta, \tau) \geq v(\lambda \eta, \tau)
$$

It follows from $(1.2 .8)$ and $(1.2 .10)$ that

$$
v(\lambda \eta, \tau)=\lambda^{1-\theta} v(\eta, \tau)
$$

and (i) is proved.

To prove (ii), fix $\eta$, and then consider the following composite map $\phi: \lambda \rightarrow \phi(\lambda)=$ $v(\lambda \eta, \tau)=\lambda^{1-\theta} v(\eta, \tau)$. The derivative of $\phi$ with respect to $\lambda$, computed in two equivalent ways one directly and one using the chain rule - is given by $\phi^{\prime}(\lambda)=$ $(1-\theta) \lambda^{-\theta} v(\eta, \tau)=\eta D_{1} v(\lambda \eta, \tau)$. For $\lambda=1$, the preceding expression becomes

$$
\phi^{\prime}(1)=(1-\theta) v(\eta, \tau)=\eta D_{1} v(\eta, \tau)
$$

which is (ii) of the lemma.

\subsubsection{The Hamilton-Jacobi-Bellman equation for the AK model}

In attempting to solve the maximization problem constituted by (1.2.6) and (1.2.7), Let $\left(c_{t}\right)_{t \geq \tau}$ be any feasible consumption program. Pick $\Delta t>0$ sufficiently small, 
apply the control $\left(c_{t}\right)_{t \geq \tau}$ during the infinitesimal time interval $(\tau, \tau+\Delta t)$, and then continue optimally afterwards from the new initial condition $\left(K_{\tau+\Delta t}, \tau+\Delta t\right)$. The present value of the stream of social welfare obtained under this action is given by

$$
e^{-\rho \tau} \frac{c_{\tau}^{1-\theta}}{1-\theta} \Delta t+o(\Delta t)+v\left(K_{\tau+\Delta t}, \tau+\Delta t\right)
$$

In (1.2.12), o( $\Delta t)$ is a term in $\Delta t$ such that $\lim _{\Delta t \rightarrow 0} o(\Delta t) / \Delta t=0$.

Because for an arbitrary feasible control $\left(c_{t}\right)_{t \geq \tau}$, such an action is not necessarily optimal, we must have

$$
e^{-\rho \tau} \frac{c_{\tau}^{1-\theta}}{1-\theta} \Delta t+o(\Delta t)+v\left(K_{\tau+\Delta t}, \tau+\Delta t\right) \leq v(\eta, \tau)
$$

with equality holding if $\left(c_{t}\right)_{t \geq \tau}$ is optimal during the infinitesimal time interval $[\tau, \tau+$ $\Delta t)$.

Let us rewrite (1.2.13) as follows

$$
e^{-\rho \tau} \frac{c_{\tau}^{1-\theta}}{1-\theta} \Delta t+o(\Delta t)+v(\eta+\Delta K, \tau+\Delta t)-v(\eta, \tau) \leq 0
$$

with equality holding if $\left(c_{t}\right)_{t \geq \tau}$ is optimal during the infinitesimal time interval $[\tau, \tau+$ $\Delta t)$. Here we have let $\Delta K=K_{\tau+\Delta t}-\eta$.

Assuming that $v$ is differentiable at $(\eta, \tau)$, we can rewrite $(1.2 .14)$ as follows:

$$
e^{-\rho \tau} \frac{c_{\tau}^{1-\theta}}{1-\theta} \Delta t+o(\Delta t)+D_{1} v(\eta, \tau) \Delta K+D_{2} v(\eta, \tau) \Delta t+o(\Delta K, \Delta t) \leq 0
$$

with equality holding if $\left(c_{t}\right)_{t \geq \tau}$ is optimal during the infinitesimal time interval $[\tau, \tau+$ $\Delta t)$. Here $o(\Delta K, \Delta t)$ is a term in $(\Delta K, \Delta t)$ such that $\lim _{(\Delta K, \Delta t) \rightarrow 0} \frac{o((\Delta K, \Delta t))}{|(\Delta K, \Delta t)|}=0$.

Dividing (1.2.15) by $\Delta t$, we obtain

$$
e^{-\rho \tau} \frac{c_{\tau}^{1-\theta}}{1-\theta}+\frac{o(\Delta t)}{\Delta t}+D_{1} v(\eta, \tau) \frac{\Delta K}{\Delta t}+D_{2} v(\eta, \tau)+\frac{o(\Delta K, \Delta t)}{\Delta t} \leq 0
$$

with equality holding if $\left(c_{t}\right)_{t \geq \tau}$ is optimal during the infinitesimal time interval $[\tau, \tau+$ $\Delta t)$. 
Now when $\Delta t \rightarrow 0,(1.2 .16)$ becomes

$$
e^{-\rho \tau} \frac{c_{\tau}^{1-\theta}}{1-\theta}+D_{1} v(\eta, \tau)\left(A \eta-c_{\tau}\right)+D_{2} v(\eta, \tau) \leq 0
$$

with equality holding if $c_{\tau}$ is the optimal consumption at time $\tau$. This result can be expressed more compactly as

$$
0=\max _{c}\left\{e^{-\rho \tau} \frac{c_{\tau}^{1-\theta}}{1-\theta}+D_{1} v(\eta, \tau)(A \eta-c)+D_{2} v(\eta, \tau)\right\}
$$

and the value of $c_{\tau}$ that solves the maximization problem in (1.2.18) is the optimal consumption at the initial condition $(\eta, \tau)$.

When the economy begins at time $t$, with $K_{t}$ as the capital stock, (1.2.18) takes on the following form, known as the Hamilton-Jacobi-Bellman equation for the Ramsey problem:

$$
0=\max _{c}\left\{e^{-\rho t} \frac{c^{1-\theta}}{1-\theta}+D_{1} v(K, t)(A K-c)+D_{2} v(K, t)\right\}
$$

and the value of $c_{t}$ that solves the maximization problem in (1.2.19) is the optimal consumption at the initial condition $\left(K_{t}, t\right)$.

\subsubsection{The current Hamiltonian}

Now because (i) the differential equation (1.2.4), which governs the motion of physical capital is autonomous; (ii) the time horizon is infinite; and (iii) time enters the objective function (1.2.5) only for discounting, we have

$$
v(K, t)=e^{-\rho t} v(K, 0),
$$

from which it follows that

$$
\begin{aligned}
& D_{1} v(K, l)=e^{-\rho t} D_{1} v(K, 0), \\
& D_{2} v(K, t)=-\rho e^{-\rho t} v(K, 0) .
\end{aligned}
$$


Using (1.2.21), in (1.2.19), we obtain the following current-value version of the Hamilton-Jacobi-Bellman equation:

$$
0=\max _{c}\left\{\frac{c^{1-\theta}}{1-\theta}+D_{1} v(K, 0)(\Lambda K-c)-\rho v(K, 0)\right\}
$$

and the value of $c$ that solves the maximization problem in $(1,2.22)$ is the optimal consumption at any instant when the capital stock at that instant is $K$.

In what follows, I shall suppress the zero in $v(K, 0)$, and simply write $v(K)$ for $v(K, 0)$. This abuse of notation allows us to rewrite (1.2.22) as

$$
0=\max _{c}\left\{\frac{c^{1-\theta}}{1-\theta}+v^{\prime}(K)(A K-c)-\rho v(K)\right\}
$$

and the value of $c$ that solves the maximization problem in (1.2.23) is the optimal consumption at any instant when the capital stock at that instant is $K$.

\subsubsection{Maximization of the current Hamiltonian}

The first-order condition that characterizes the maximization problem on the right side of $(1.2 .22)$ is given by

$$
c^{-\theta}-v^{\prime}(K)=0
$$

from which we obtain

$$
c=\left(v^{\prime}(K)\right)^{-\frac{1}{\theta}}
$$

Using (1.2.25) in (1.2.23), we obtain

$$
\begin{aligned}
0 & =\frac{\left(v^{\prime}(K)\right)^{-\frac{1-\theta}{\theta}}}{1-\theta}+v^{\prime}(K)\left(A K-\left(v^{\prime}(K)\right)^{-\frac{1}{\theta}}\right)-\rho v(K) \\
& ==\frac{\left(v^{\prime}(K)\right)^{1-\frac{1}{\theta}}}{1-\theta}+v^{\prime}(K)\left(A K-\left(v^{\prime}(K)\right)^{-\frac{1}{\theta}}\right)-\rho v(K) \\
& =\frac{\theta}{1-\theta}\left(v^{\prime}(K)\right)^{1-\frac{1}{\theta}}+v^{\prime}(K)(A K)-\rho v(K)
\end{aligned}
$$




\subsubsection{The closed-form solution of the Hamilton-Jacobi-Bellman equation for the AK model}

Using (ii) of the lemma in (1.2.26), we obtain

$$
0=\frac{\theta}{1-\theta}\left(v^{\prime}(K)\right)^{1-\frac{1}{\theta}}+v^{\prime}(K)(A K)-\frac{\rho}{1-\theta} K v^{\prime}(K),
$$

which can be reduced to

$$
0=\frac{\theta}{1-\theta} v^{\prime}(K)^{-\frac{1}{\theta}}+(A(1-\theta)-\rho) /(1-\theta) K
$$

or

$$
v^{\prime}(K)=\left(\frac{1}{\theta}(\rho-A(1-\theta))\right)^{-\theta} K^{-\theta}
$$

Integrating (1.2.29), and using the boundary condition $v(0)=0$, we obtain the following closed-form expression of the optimal-value function for the AK model:

$$
v(K)=\frac{1}{1-\theta}\left(\frac{1}{\theta}(\rho-A(1-\theta))\right)^{-\theta} K^{1-\theta} .
$$

I summarize the result just obtained in the following proposition:

Proposition 1. The closed-form optimal-value function for the AK model is

$$
v(K)=\frac{1}{1-\theta}\left(\frac{1}{\theta}(\rho-A(1-\theta))\right)^{-\theta} K^{1-\theta} .
$$

\subsubsection{Balanced growth}

Using (1.2.25) and(1.2.30), we obtain the following expression for the optimal consumption at each instant as a function of the capital stock:

$$
c=v^{\prime}(K)^{-\frac{1}{\theta}}=\left(\frac{1}{\theta}(\rho-A(1-\theta))\right)^{-\theta} K .
$$

That is, at each time $t$, the optimal consumption as a function of the capital stock is given by

$$
c_{t}=\left(\frac{\theta}{\rho-A(1-\theta)}\right)^{\theta} K_{t}
$$


The following restriction must be imposed upon the parameters so that consumption is positive at each instant:

$$
\rho>(1-\theta) A
$$

In the literature of economic growth theory the parameter A represents the real interest rate in this model. The (1.2.33)condition can be interpreted as the impatience condition that we imposed in the infinite horizon perfect foresight consumption model. The motion of the capital stock is governed by the following differential equation:

$$
\begin{aligned}
\frac{d K}{d t} & =A K_{t}-c_{t} \\
& =\left(A-\left(\frac{\theta}{\rho-A(1-\theta)}\right)^{\theta}\right) K_{t}
\end{aligned}
$$

which indicates that the capital stock grows exponentially through time at the constant rate

$$
\gamma=A-\left(\frac{\theta}{\rho-A(1-\theta)}\right)^{\theta} .
$$

In order for the economy to grow, the following restriction must be imposed upon the parameters:

$$
\left(\frac{\theta}{\rho-A(1-\theta)}\right)^{\theta}<A
$$

Together,(1.2.3.3) and (1.2.36) define the non-empty interval in which, $\rho$, the rate of time preference is located:

$$
(1-\theta) A<\rho<(1-\theta) A+\frac{A^{\frac{1}{\theta}}}{\theta} .
$$

When $\theta=1$, we find the most common assumption in the literature of endogenous growth:

$$
0<\rho<A
$$

It ensures a positive growth rate of consumption (for example, Lucas (1988), Jones and Manuelli (1990), and Rebelo (1991)). Thus, when the conditions (1.2.33) and 
(1.2.36) are both met, the economy grows along a balanced growth path, with the capital stock and consumption grow at the same rate. There is no transition to the steady state: the economy enters the steady state of balanced growth immediately at time 0 .

\subsection{Economic growth with exhaustible resources}

\subsubsection{Formal statement of the problem}

In the model, time is continuous and denoted by $t, t \geq 0$. The economy produces a consumption good, using capital and an exhaustible resource. The consumption good can also be used as investment goods to augment the stock of capital.

Let $u(c)$ denote the instantaneous utility of consumption, I assume that

$$
u(c)=\frac{c^{1-\theta}}{1-\theta}
$$

where $\theta, 0<\theta<1$, is a parameter, which is the inverse of the inter-temporal elasticity of substitution.

The consumption good is produced according to the following Cobb-Douglas technology:

$$
Y=A K^{\alpha} x^{1-\alpha}
$$

where $Y$ represents output, $K$ the capital input, and $x$ the exhaustible resource input. Also, $A$ is the technological level of the economy, and $\alpha$ is a parameter, with $0<\alpha<1$.

Let $X_{0}>0$ denote the initial stock of the exhaustible resource and $X_{t}$ denote the remaining resource stock at time $t, t \geq 0$. An extraction program is a non-negative, piecewise, and right continuous function $t \rightarrow x_{t}, t \geq 0$. An extraction program $t \rightarrow$ 
$x_{t}, t \geq 0$, is feasible if it satisfies the following stock constraint:

$$
\int_{0}^{\infty} x_{t} d t \leq X_{0}
$$

The stock constraint (1.3.3) can be expressed in the following equivalent differential form:

$$
\begin{aligned}
\frac{d X}{d t} & =-x_{t} \\
X_{0} & >\text { 0is given. }
\end{aligned}
$$

I assume that capital does not depreciate. By a consumption program, I mean a non-negative piece-wise and right-continuous function $t \rightarrow c_{t}, t \geq 0$, where $c_{t}$ is the consumption at time $t$. Let $K_{t}$ denote the capital stock at time $t$. The motion of the stock of capital is governed by the following differential equation:

$$
\begin{aligned}
& \frac{d K}{d t}=A K_{t}^{\alpha} x_{t}^{1-\alpha}-c_{t}, \\
& K_{0}>0 \text { is given. }
\end{aligned}
$$

A consumption program is said to be feasible if the solution of (1.3.6) is such that $K_{t} \geq 0$ for all $\iota \geq 0$. Let $\left(c_{t}\right)_{t \geq 0}$ be a feasible consumption program. The discounted value of the stream of social welfare associated with $\left(c_{t}\right)_{t \geq 0}$ is given by

$$
\int_{0}^{\infty} e^{-\rho t} u\left(c_{t}\right) d t
$$

In (1.3.7), $\rho>0$ is the rate of time preference. The problem faced by the central planner is to find a feasible extraction program $\left(x_{t}\right)_{t \geq 0}$ and a feasible consumption program $\left(c_{t}\right)_{t \geq 0}$ to maximize (1.3.7) subject to (1.3.5) and (1.3.6).

\subsubsection{The optimal-value function}

Suppose that the economy begins at time $\tau \geq 0$ with $\xi$ as the remaining stock of oil and $\eta$ as the stock of capital. Let

$$
v(\xi, \eta, \tau)=\max _{\left(x_{t}, c_{t}\right)_{t \geq \tau}} \int_{\tau}^{\infty} e^{-\rho t} u\left(c_{t}\right) d t
$$


subject to

$$
\begin{gathered}
\frac{d X}{d t}=-x_{t}, \\
X(\tau)=\xi, \\
\frac{d K}{d t}=A K_{t}^{\alpha} x_{t}^{1-\alpha}-c_{t}, \\
K_{\tau}=\eta .
\end{gathered}
$$

As defined, $v(\xi, \eta, \tau)$ represents the optimal present value of social welfare, given that the economy begins at time $\tau$ with $\xi$ as the remaining stock of oil and $\eta$ as the stock of capital. Note that when $\xi=X_{0}, \eta=K_{0}$, the preceding dynamic optimization problem is reduced to the central planner's original problem. The optimal-value function has the following obvious properties. First, it is increasing in the stock of fossil fuels as well as the capital stock. Second, when the economy has a positive capital stock $(\eta>0)$, but no oil $(\xi=0)$, the only option available to the central planner is to consume the capital stock, and then cease to exist. Moreover, since the return function is concave and the constraint is linear, the value function is concave and there is a unique global optimum, Caputo (2005). The discounted social welfare obtained under this scenario is $v(0, \eta, \tau)>0$. Third, when the economy has a positive stock of fossil fuels $(\xi>0)$, but no capital $(\eta=0)$, there is no production. Furthermore, because oil is not a consumption good, the discounted social welfare will be $v(\xi, 0, \tau)=0$, and the economy cannot exist. The following lemma presents an important property of the optimal-value function that I shall use to solve the Hamilton-Jacobi-Bellman equation:

Lemma 2. For any $\tau$, the function $v(\cdot, \tau):(\xi, \eta) \rightarrow v(\xi, \eta, \tau)$ is homogeneous of degree $1-\theta$ in $(\xi, \eta)$. That is, for any $\lambda>0$, we have $v(\lambda \xi, \lambda \eta, \tau)=\lambda^{1-\theta} v(\xi, \eta, \tau)$.

Proof. This lemma can be proved in the same manner as Lemma 1. 
Suppose that the economy begins at time $t$, with $X$ as the remaining stock of oil and $K$ as the stock of capital. I drop the time subscript from the $\mathrm{K}, \mathrm{x}$ and $\mathrm{c}$ unless it is ambiguous. In the appendix, I derive the following Hamilton-Jacobi-Bellman equation, which must be satisfied by the optimal-value function $v$ at each point $(X, K, t)$ :

$$
\begin{array}{r}
0=\max _{(x, c)}\left\{e^{-\rho t} \frac{c^{1-\theta}}{1-\theta}-D_{1} v(X, K, t) x+D_{2} v(X, K, t)\left(A K^{\alpha} x^{1-\alpha}-c\right)\right. \\
\left.+D_{3} v(X, K, t)\right\} .
\end{array}
$$

As in the AK model, we have $v(X, K, t)=e^{-p t} v(X, K, 0)$, and this means $D_{1} v(X, K, t)=e^{-\rho t} D_{1} v(X, K, 0), D_{2} v(X, K, t)=e^{-\rho t} D_{2} v(X, K, 0)$, and $D_{3} v(X, K, t)=-\rho e^{-\rho t} v(X, K, 0)$.

In what follows, we shall suppress the zero in $v(X, K, 0)$ and write it simply as $v(X, K)$. This abuse of notation allows us to rewrite (1.3.11) under the current-value form:

$$
0=\max _{(x, c)}\left\{\frac{c^{1-\theta}}{1-\theta}-D_{1} v(X, K) x+D_{2} v(X, K)\left(A K^{\alpha} x^{1-\alpha}-c\right)-\rho v(X, K)\right\}
$$

Proposition 2. For any $X \geq 0, K \geq 0$, the optimal-value function has the following form:

$$
v\left(X, K^{*}\right)=K^{1-\theta} \omega\left(\frac{X}{K}\right),
$$

where $\omega(\cdot)$ is an increasing function.

Proof. According to Lemma $2, v(X, K)$ is homogeneous of degree $1-\theta$ in $(X, K)$. 
Thus, for $K>0$, we have

$$
\begin{aligned}
v(X, K) & =v\left(K\left(\frac{X}{K}, 1\right)\right) \\
& =K^{1-\theta} v\left(\frac{X}{K}, 1\right) \\
& =K^{1-\theta} \omega\left(\frac{X}{K}\right)
\end{aligned}
$$

where I define $\omega: \lambda \rightarrow \omega(\lambda)=v(\lambda, 1), \lambda \geq 0$. Furthermore, because $v(X, K)$ is increasing in $X$ for each given $K>0$, it must be the case that $\omega(\lambda)$ is increasing in $\lambda$, and thus Proposition 2 is proved.

\subsubsection{The optimal-value function when the economy has cap- ital but no fossil fuels}

When there are no fossil fuels, the Hamilton-Jacobi-Bellman equation (1.3.12) is reduced to

$$
0=\max _{c}\left\{\frac{c^{1-\theta}}{1-\theta}+D_{2} v(0, K)(-c)-\rho v(0, K)\right\}
$$

If I suppress 0 in $v(0, K)$ and write $v(0, K)$ simply as $v(K),(1.3 .14)$ becomes

$$
0=\max _{c}\left\{\frac{c^{1-\theta}}{1-\theta}+v^{\prime}(K)(-c)-\rho v(K)\right\} .
$$

The following first-order condition characterizes the optimal consumption when $K$ is the capital stock

$$
0=c^{-\theta}-v^{\prime}(K)
$$

from which we obtain

$$
\begin{aligned}
0 & =\frac{1}{1-\theta} v^{\prime}(K)^{-\frac{1-\theta}{\theta}}+v^{\prime}(K)\left(-v^{\prime}(K)^{-\frac{1}{\theta}}\right)-\rho v(K) \\
& =\frac{\theta}{1-\theta} v^{\prime}(K)^{-\frac{1-\theta}{\theta}}-\rho v(K)
\end{aligned}
$$


Using (1.3.17) in (1.3.15), we have

$$
v(K)^{\frac{\theta}{1-\theta}} v^{\prime}(K)=\left(\frac{\rho(1-\theta)}{\theta}\right)^{-\frac{\theta}{1-\theta}} .
$$

Equation (1.3.18) can be rewritten as the following differential equation

$$
v(K)^{\frac{\theta}{1-\theta}} v^{\prime}(K)=\left(\frac{\rho(1-\theta)}{\theta}\right)^{-\frac{\theta}{1-\theta}} .
$$

Integrating (1.3.19), we obtain

$$
(1-\theta) v(K)^{\frac{1}{1-\theta}}=\left(\frac{\rho(1-\theta)}{\theta}\right)^{-\frac{\theta}{1-\theta}} K+\text { constan } l .
$$

The constant of integration is 0 because of the boundary condition $v(0)=0$. Thus, the optimal-value function for the case of no fossil fuels is

$$
v(K)=\frac{1}{1-\theta}\left(\frac{\rho}{\theta}\right)^{-\theta} K^{1-\theta}
$$

I state more formally the result just obtained in the following proposition:

Proposition 3. When the economy has a positive stock of capital, but no oil, the optimal-value function for consuming the capital stock is

$$
v(K)=\frac{1}{1-\theta}\left(\frac{\rho}{\theta}\right)^{-\theta} K^{1-\theta}
$$

It follows from (1.3.17) and (1.3.21) that the optimal consumption as a function of the capital stock is given by

$$
c=v^{\prime}(K)^{-\frac{1}{\theta}}=\frac{\rho}{\theta} K
$$

which is proportional to the capital stock.

The motion of the remaining capital stock is governed by the following differential equation:

$$
\frac{d K}{d t}=-\frac{\rho}{\theta} K_{t}, \quad K_{0} \text { is given }
$$


The solution of $(1.3 .23)$ is

$$
K_{t}=K_{0} e^{-\frac{\rho}{\theta} t}
$$

That is, the capital stock declines exponentially through time at a constant rate, and so does consumption.

\subsubsection{The optimal-value function when the economy has both capital and fossil fuels}

In this section I will illustrate the use of the homogeneity property of the value function to derive the closed form solution for the Bellman equation. According to (1.3.13) and (1.3.21), we have:

$$
v(0, K)=K^{1-\theta} \omega(0)=\frac{1}{1-\theta}\left(\frac{\rho}{\theta}\right)^{-\theta} K^{1-\theta}
$$

from which we obtain

$$
\omega(0)=\frac{1}{1-\theta}\left(\frac{\rho}{\theta}\right)^{-\theta}
$$

Using (1.3.13), we obtain the following expressions for the current shadow prices of oil and capital

$$
\begin{aligned}
& D_{1} v(X, K)=K^{-\theta} \omega^{\prime}\left(\frac{X}{K}\right) \\
& D_{2} v(X, K)=K^{-\theta}(1-\theta) \omega\left(\frac{X}{K}\right)-K^{-1-\theta} X \omega^{\prime}\left(\frac{X}{K}\right) .
\end{aligned}
$$

Using (1.3.13) and (1.3.26) in (1.3.12), we obtain the following version of the current Hamilton-Jacobi-Bellman equation

$$
\begin{aligned}
0=\max _{(x, c)}\{ & \frac{1}{1-\theta} c^{1-\theta}-K^{-\theta} \omega^{\prime}\left(\frac{X}{K}\right) x \\
& +\left(K^{-\theta}(1-\theta) \omega\left(\frac{X}{K}\right)-K^{-1-\theta} X \omega^{\prime}\left(\frac{X}{K}\right)\right)\left(A K^{\alpha} x^{1-\alpha}-c\right) \\
& \left.-\rho K^{1-\theta} \omega\left(\frac{X}{K}\right)\right\} .
\end{aligned}
$$


Now let $\lambda=X / K$. Substituting $X=\lambda K$ in (1.3.27), we obtain the following version of the current Hamilton-Jacobi-Bellman equation in terms of the capital stock $K^{-}$and the resource endowment/capital ratio:

$$
\begin{aligned}
0=\max _{(x, c)}\left\{\frac{c^{1-\theta}}{1-\theta}-\right. & K^{1-\theta} \rho \omega(\lambda)-K^{-\theta} x \omega^{\prime}(\lambda) \\
& \left.+\left(A K^{\alpha} x^{1-\alpha}-c\right)\left(K^{-\theta}(1-\theta) \omega(\lambda)-K^{-\theta} \lambda \omega^{\prime}(\lambda)\right)\right\} .
\end{aligned}
$$

The following first-order condition characterizes the value of $c$ that solves the maximization problem in (1.3.28):

$$
c^{-\theta}-K^{-\theta}(1-\theta) \omega(\lambda)+K^{-\theta} \lambda \omega^{\prime}(\lambda)=0
$$

from which we obtain

$$
c=K\left(\omega(\lambda)-\theta \omega(\lambda)-\lambda \omega^{\prime}(\lambda)\right)^{-1 / \theta}
$$

The following first-order condition characterizes the value of $x$ that solves the maximization problem in (1.3.27):

$$
-K^{-\theta} \omega^{\prime}(\lambda)+A K^{\alpha} x^{-\alpha}(1-\alpha)\left(K^{-\theta}(1-\theta) \omega(\lambda)-K^{-\theta} \lambda \omega^{\prime}(\lambda)\right)=0
$$

from which we obtain

$$
x=A^{\frac{1}{\alpha}} K(1-\alpha)^{\frac{1}{\alpha}} \omega^{\prime}(\lambda)^{-1 / \alpha}\left((1-\theta) \omega(\lambda)-\lambda \omega^{\prime}(\lambda)\right)^{\frac{1}{\alpha}} .
$$

Using (1.3.30) and (1.3.32) in (1.3.18), we obtain - with the symbolic computation power of Mathematica - after many manipulations (see the appendix)

$$
\begin{aligned}
0= & \frac{1}{((1-\alpha)(1-\theta)) K^{1-\theta} \omega^{\prime}(\lambda)^{-\frac{1}{\alpha}}\left(\omega(\lambda)-\theta \omega(\lambda)-\lambda \omega^{\prime}(\lambda)\right)^{-\frac{1}{\theta}}} \times \\
& -\left((1-\alpha) \theta \lambda \omega^{\prime}(\lambda)^{1+\frac{1}{\alpha}}-A^{\frac{1}{\alpha}}(1-\alpha)^{\frac{1}{\alpha}} \alpha(1-\theta) \omega^{\prime}(\lambda)\left(\omega(\lambda)-\theta \omega(\lambda)-\lambda \omega^{\prime}(\lambda)\right)^{\frac{1}{\alpha}+\frac{1}{\theta}}\right. \\
& \left.+(1-\alpha)(1-\theta) \omega(\lambda) \omega^{\prime}(\lambda)^{\frac{1}{\alpha}}\left(\theta-\rho\left(\omega(\lambda)-\theta \omega(\lambda)-\lambda \omega^{\prime}(\lambda)\right)^{\frac{1}{\theta}}\right)\right) .
\end{aligned}
$$


Observe that the right side of (1.3.33) is the product of

$$
\frac{1}{((1-\alpha)(1-\theta)) K^{1-\theta} \omega^{\prime}(\lambda)^{-\frac{1}{\alpha}}\left(\omega(\lambda)-\theta \omega(\lambda)-\lambda \omega^{\prime}(\lambda)\right)^{-\frac{1}{\theta}}}
$$

and

$$
\begin{gathered}
-(1-\alpha) \theta \lambda \omega^{\prime}(\lambda)^{1+\frac{1}{\alpha}}- \\
A^{\frac{1}{\alpha}}(1-\alpha)^{\frac{1}{\alpha}} \alpha(1-\theta) \omega^{\prime}(\lambda)\left(\omega(\lambda)-\theta \omega(\lambda)-\lambda \omega^{\prime}(\lambda)\right)^{\frac{1}{\alpha}+\frac{1}{\theta}}+ \\
(1-\alpha)(1-\theta) \omega(\lambda) \omega^{\prime}(\lambda)^{\frac{1}{\alpha}}\left(\theta-\rho\left(\omega(\lambda)-\theta \omega(\lambda)-\lambda \omega^{\prime}(\lambda)\right)^{\frac{1}{\theta}}\right)
\end{gathered}
$$

Now we know that $\omega^{\prime}(\lambda)>0, K>0$, and in order for $\left(\omega(\lambda)-\theta \omega(\lambda)-\lambda \omega^{\prime}(\lambda)\right)^{-1 / \theta}$ not to be infinite (see (1.3.30)), we must also have $\omega(\lambda)-\theta \omega(\lambda)-\lambda \omega^{\prime}(\lambda) \neq 0$. Hence (1.2.32) will hold if

$$
\begin{aligned}
0= & -(1-\alpha) \theta \lambda \omega^{\prime}(\lambda)^{1+\frac{1}{\alpha}}+A^{\frac{1}{\alpha}}(1-\alpha)^{\frac{1}{\alpha}} \alpha(1-\theta) \omega^{\prime}(\lambda)\left(\omega(\lambda)-\theta \omega(\lambda)-\lambda \omega^{\prime}(\lambda)\right)^{\frac{1}{\alpha}+\frac{1}{\theta}} \\
& +(1-\alpha)(1-\theta) \omega(\lambda) \omega^{\prime}(\lambda)^{\frac{1}{\alpha}}\left(\theta-\rho\left(\omega(\lambda)-\theta \omega(\lambda)-\lambda \omega^{\prime}(\lambda)\right)^{\frac{1}{\theta}}\right) .
\end{aligned}
$$

The following proposition is immediate:

Proposition 4. The function $\omega(\lambda), \lambda \geq 0$, satisfies the following differential equation:

$$
\begin{aligned}
0= & -(1-\alpha) \theta \lambda \omega^{\prime}(\lambda)^{1+\frac{1}{\alpha}}+A^{\frac{1}{\alpha}}(1-\alpha)^{\frac{1}{\alpha}} \alpha(1-\theta) \omega^{\prime}(\lambda)\left(\omega(\lambda)-\theta \omega(\lambda)-\lambda \omega^{\prime}(\lambda)\right)^{\frac{1}{\alpha}+\frac{1}{\theta}} \\
& -(1-\alpha)(-1+\theta) \omega(\lambda) \omega^{\prime}(\lambda)^{\frac{1}{\alpha}}\left(\theta-\rho\left(\omega(\lambda)-\theta \omega(\lambda)-\lambda \omega^{\prime}(\lambda)\right)^{\frac{1}{\theta}}\right) .
\end{aligned}
$$

with the initial condition

$$
\omega(0)=\frac{1}{1-\theta}\left(\frac{\rho}{\theta}\right)^{-\theta}
$$

The differential equation stated in Proposition 2 is highly non-linear, and cannot be put into the conventional form of an ordinary differential equation presented in textbooks on the subject. So, to find the solution of this equation I guess that it satisfies the following condition: 


$$
0=\left(\theta-\rho\left(\omega(\lambda)-\theta \omega(\lambda)-\lambda \omega^{\prime}(\lambda)\right)^{\frac{1}{\theta}}\right)
$$

which can be rewritten as

$$
\omega^{\prime}(\lambda)=\frac{1}{\lambda}\left(-\left(\frac{\theta}{\rho}\right)^{\theta}+\omega(\lambda)(1-\theta)\right) .
$$

The solution of (1.3.36) is given by

$$
\omega(\lambda)=\frac{\left(\frac{\theta}{\rho}\right)^{\theta}}{1-\theta}+b \lambda^{1-\theta},
$$

where $b$ is the constant of integration.

Note that when $\lambda=0,(1.3 .36)$ is reduced to

$$
\omega(0)=\frac{1}{1-\theta}\left(\frac{\rho}{\theta}\right)^{-\theta}
$$

that is, the guessed solution for $\omega(\lambda)$ satisfies the initial condition (1.3.25).

To find the constant of integration $b$, substitute (1.3.37) in (1.3.34) to obtain

$$
0=\lambda^{-\theta} b\left(-b^{\frac{1}{\alpha}}(1-\alpha)(1-\theta)^{1+\frac{1}{\alpha}} \theta \lambda^{1-\frac{\theta}{\alpha}}+A^{\frac{1}{\alpha}}(1-\alpha)^{\frac{1}{\alpha}} \alpha(1-\theta)^{2} \theta^{\left(\frac{1}{\alpha}+\frac{1}{\theta}\right) \theta} \rho^{-\left(\frac{1}{\alpha}+\frac{1}{\theta}\right) \theta}\right) .
$$

Now note that if (1.3.37) is the correct functional form for $\omega(\lambda)$, then (1.3.39) must hold for any value of $\lambda>0$. Also, note that the expression inside the pair of grand parentheses in (1.3.39) contains $\lambda^{1-\frac{\theta}{\alpha}}$. Hence in order for the right side of (1.3.39) to be independent of $\lambda$, I must assume that $\alpha=\theta$. Using this assumption, I can simplify (1.3.39) as

$$
0=\lambda^{-\theta} b\left(\frac{1}{\rho^{2}} A^{\frac{1}{\theta}}(1-\theta)^{\frac{1}{\theta}}(1-\theta)^{2} \theta^{3}-(1-\theta)^{2+\frac{1}{\theta}} \theta b^{\frac{1}{\theta}}\right) .
$$

The correct value for the constant of integration is the value of $b>0$ that solves (1.3.40), and this value of $b$ is given by

$$
b=A\left(\frac{\theta}{\rho}\right)^{2 \theta} .
$$

I summarize the results just obtained in the following proposition: 
Proposition 5. Suppose that $\alpha=\theta$, i.e., the share of capital in national income is equal to the inverse of the inter-temporal elasticity of substitution. Then the closedform expression of the optimal-value function for the model of economic growth with exhaustible resources is given by

$$
\begin{aligned}
v(X, K) & =K^{1-\theta} \omega\left(\frac{X}{K}\right) \\
& =K^{1-\theta}\left(\frac{1}{1-\theta}\left(\frac{\rho}{\theta}\right)^{-\theta}+A\left(\frac{\theta}{\rho}\right)^{2 \theta}\left(\frac{X}{K}\right)^{1-\theta}\right) \\
& =\frac{1}{1-\theta}\left(\frac{\theta}{\rho}\right)^{\theta} K^{1-\theta}+A\left(\frac{\theta}{\rho}\right)^{2 \theta} X^{1-\theta} .
\end{aligned}
$$

\subsubsection{The time paths of resource depletion, capital accumu- lation, and consumption}

Using the closed-form expression for the optimal-value function, as represented by (1.3.6), in (1.2.29), we obtain the following expression for the optimal consumption:

$$
c_{t}=\frac{\rho}{\theta} K_{t}
$$

That is, at any instant, the optimal consumption is a constant proportion of the capital stock.

Using the closed-form expression for the optimal-value function, as represented by (1.3.42), in (1.3.32), we obtain the following expression for the optimal extraction rate:

$$
x_{t}=\frac{\rho}{\theta} X_{t}
$$

That is, at any instant, the optimal extraction rate is a constant proportion of the remaining oil stock, and the constant of proportionality is the same as that for the optimal consumption.

It follows from (1.3.44) that the motion of the remaining stock of oil is governed 
by the following differential equation:

$$
\begin{aligned}
& \frac{d X}{d t}=-\frac{\rho}{\theta} X_{t}, \\
& X_{0}>0 \text { is given. }
\end{aligned}
$$

The solution of (1.3.45) is

$$
X_{t}=X_{0} e^{-\frac{\rho}{\theta} t}
$$

That is, the remaining oil stock declines exponentially through time at the constant rate $\frac{\rho}{\theta}$.

The motion of the capital stock is governed by the following differential equation:

$$
\begin{aligned}
& \frac{d K}{d t}=-\frac{\rho}{\theta} K_{t}+A\left(\frac{\rho}{\theta} X_{0} e^{-\frac{t \rho}{\theta}}\right)^{1-\alpha} K_{t}^{\alpha}, \\
& K_{0}>0 \text { is given. }
\end{aligned}
$$

The solution of the differential equation (1.3.47) is

$$
\begin{aligned}
K_{t} & =\left(e^{-\frac{t(1-\theta) \rho}{\theta}} K_{0}^{1-\theta}+A e^{-\frac{t(1-\theta) \rho}{\theta}} t X_{0}^{1-\theta} \theta^{-1+\theta} \rho^{1-\theta}-A e^{-\frac{t(1-\theta) \rho}{\theta}} t X_{0}^{1-\theta} \theta^{\theta} \rho^{1-\theta}\right)^{\frac{1}{1-\theta}} \\
& =\left(e^{-\frac{t(1-\theta) \rho}{\theta}} K_{0}^{1-\theta}+A e^{-\frac{t(1-\theta) \rho}{\theta}} t X_{0}^{1-\theta} \theta^{\theta} \rho^{1-\theta}\left(\frac{1-\theta}{\theta}\right)\right)^{\frac{1}{1-\theta}} \\
& =e^{-\frac{\rho}{\theta} t}\left(K_{0}^{1-\theta}+A t X_{0}^{1-\theta} \theta^{\theta} \rho^{1-\theta}\left(\frac{1-\theta}{\theta}\right)\right)^{\frac{1}{1-\theta}} .
\end{aligned}
$$

I summarize the results just obtained in the following proposition:

Proposition 6. Along the optimal trajectory, consumption is Proportional to the capital stock and the oil extraction rate is proportional to the remaining oil stock, with the same proportionality constant as that for consumption. Furthermore, In the long run, the capital stock asymptotically approaches 0 , and this means that the economy is not sustainable. 
Benchekroun and Withagen (2010) observed that "Pezzey and Withagen (1998) and Hartwick et al. (2003) have shown without explicitly finding the solution that consumption can be single peaked". This question can easily be answered by logarithmically differentiating (1.3.48) with respect to $t$ :

$$
\frac{1}{K_{l}} \frac{d K}{d t}=-\frac{\rho}{\theta}+\frac{A \theta^{\theta} \rho K_{0}^{\theta} X_{0}}{A t(1-\theta) \theta^{\theta} \rho K_{0}^{\theta} X_{0}+\theta \rho^{\theta} K_{0} X_{0}^{\theta}}
$$

Observe that the denominator of the second expression on the right side of $(1.3 .49)$ is strictly positive and decreasing in $t$. Thus, when $t$ is large enough, $K_{t}$ will decline exponentially to 0 at rate $\frac{\rho}{\theta}$. Hence if

$$
-\frac{\rho}{\theta}+\frac{A \theta^{\theta} \rho K_{0}^{\theta} X_{0}}{\theta \rho^{\theta} K_{0} X_{0}^{\theta}}=-\frac{\rho}{\theta}+A \theta^{\theta-1} \rho^{1-\theta} K_{0}^{\theta-1} X_{0}^{1-\theta} \leq 0
$$

or, equivalently, if

$$
\left(\frac{X_{0}}{K_{0}}\right)^{1-\theta} \leq \frac{1}{A}\left(\frac{\rho}{\theta}\right)^{\theta}
$$

then $d K / d \iota<0$ for all $\iota>0$. On the other hand, if the inequality (1.3.51) is reverse, then (1.3.49) vanishes at the instant $\hat{\imath}$ given by

$$
\hat{t}=\frac{\theta^{1-\theta} K_{0}^{-\theta}\left(A \theta^{\theta} K_{0}^{\theta} X_{0}-\rho^{\theta} K_{0} X_{0}^{\theta}\right)}{A(1-\theta) \rho X_{0}}>0
$$

I state formally the result just obtained in the following proposition:

Proposition 7. If

$$
\left(\frac{X_{0}}{K_{0}}\right)^{1-\theta} \leq \frac{1}{A}\left(\frac{\rho}{\theta}\right)^{\theta}
$$

then the capital stock falls along the optimal trajectory. On the other hand, if the inequality (1.3.50) is reversed, then the capital stock first rises, reaches a maximum at time

$$
\hat{t}=\frac{\theta^{1-\theta} K_{0}^{-\theta}\left(A \theta^{\theta} K_{0}^{\theta} X_{0}-\rho^{\theta} K_{0} X_{0}^{\theta}\right)}{A(1-\theta) \rho X_{0}}>0
$$

and then declines to 0 asymptotically as $t \rightarrow \infty$. 
Now recall from (1.3.43) that along the optimal trajectory, consumption is proportional to the capital stock. Using this result and Proposition (7), I obtain the following pattern of consumption

Corollary 1. If

$$
\left(\frac{X_{0}}{K_{0}}\right)^{1-\theta} \leq \frac{1}{A}\left(\frac{\rho}{\theta}\right)^{\theta}
$$

then consumption is monotonically declining through time. On the other hand, if the inequality (1.3.14) is reversed, then consumption first rises, reaches a peak at time

$$
\hat{t}=\frac{\theta^{1-\theta} K_{0}^{-\theta}\left(A \theta^{\theta} K_{0}^{\theta} X_{0}-\rho^{\theta} K_{0} X_{0}^{\theta}\right)}{A(1-\theta) \rho X_{0}}>0
$$

and then declines to 0 asymptotically as $t \rightarrow \infty$.

\subsection{Concluding remarks}

The traditional approach to solving models of economic growth is to invoke the maximum principle. Very seldom is the closed-form solution obtained in this manner. Instead, the analysis concentrates mostly on the steady state, and the transition to the steady state, whenever it is analyzed, is carried out with the help of a phase diagram. The lack of closed-form solution to the model does not allow for many interesting questions to be analyzed. Recently, some researchers have attempted to find the closed-form solutions to the differential equations that govern the motion of the state and co-state variables, and thus made it possible to study the solution of economic growth models in depth.

In this paper, I have adopted an alternative approach by showing that the homogeneity of the Bellman function can be exploited to find its closed form. The closed-form Bellman function, in addition to the economic contents that it embodies, 
allows a more direct and in-depth analysis of the optimal solution. I illustrated the method by solving two economic growth models. The first model is the AK model of endogenous growth. The second model is the well known model of growth with exhaustible resources of Dasgupta and Heal (1974). For the second model, I have made the special assumption that the share of capital is equal to the inverse of the inter-temporal elasticity of substitution. I admit that this is a very restrictive assumption, but the approach might represent the first step in finding the closed form for the Bellman function of more complicated model. 


\section{References}

[1] Alvarez, F., N. L. Stokey (1998): "Dynamic Programming with Homogeneous Functions," Journal of Economic Theory, 82:167-189.

[2] Benchekroun, H., C. Withagen (2010): "The Optimal Depletion of Exhaustible Resources: a Complete Characterization," Resources and Energy Economics, 33:612 636.

[3] Caputo, M.R.(2005): "Foundations of Dynamic Economic Analysis: Optimal Control Theory and Applications," Cambridge University Press, Cambridge

[4] Dasgupta, P., G. M. Heal (1974): "The Optimal Depletion of Exhaustible Resources," Review of Economic Studies, 41:3 28.

[5] Guerrini, L. (2010): "A Closed-Form Solution to the Ramsey Model with the von Bertalanffy Population Law," Applied Mathematical Sciences, 4:3239 3244.

[6] Hartwick, J., N. Van Long, H. Tian (2003): "On the Peaking of Consumption with Exhaustible Resoutces and Zero Net Investment," Environmental and Resource Economics, 24:235 244.

[7] Jones, L. E., R. Manuelli (1990): "A Convex Model of Equilibrium Growth: Theory and Policy Implications," Journal of Political Economy, 98:1008-1038. 
[8] Lucas, R. E., Jr. (1988): "On the Mechanics of Economic Development," Journal of Monetary Economics, 22:3 42.

[9] Pezzey, J., C. Withagen (1998): "The Rise, Fall and Sustainability of CapitalResource Economies," Scandinavian Journal of Economics, 100:513 527.

[10] Pontryagin L. S. , Boltyanskii V. G., Gamkrelidze R. V., Mishchenko E. F. (1962): "The Mathematical Theory of Optimal Processes," English translation, translated from Russian by K. N. Trirogoff, Interscience Publishers.

[11] Rebelo, S. (1991): "Long Run Policy Analysis and Long Run Growth," Journal of Political Economy, 99:500 -521.

[12] Ruiz-Tamarit, J. R. (2008): "The Closed-Form Solution for a Family of Fourdimension Nonlinear MHDS," Journal of Economic Dynamics and Control, $32: 10001014$

[13] Smith, T. W. (2006): "An Analytical Solution to the Ramsey Model," Contributions to Macroeconomics, 6:1-27.

[14] Solow, R. M. (1974): "Intergenerational Equity and Exhaustible Resources," Review of Economic Studies, 41:29 45.

[15] Stiglitz, J. (1974): "Growth with Exhaustible Natural Resources: Efficient and Optimal Growth Paths," Review of Economic Studies, 41:123 137.

[16] Stokey, N. L. (1998): "Are There Limits to Growth?," International Economic Review, 39:1 31 . 


\section{Appendix 1.A Derivation of the HJB equation and the adjoint equations}

Let $t \rightarrow\left(x_{t}, c_{t}\right), t \geq \tau$, be an admissible control policy. Now consider an infinitesimal time interval $[\tau, \tau+\Delta t$ ). If we carry out this control policy during the infinitesimal time interval $[\tau, \tau+\Delta t)$, and then switch to the optimal control policy from time $\tau+\Delta t$ on, then the discounted utilities obtained under this action are given by $e^{-p \tau} u\left(c_{\tau}\right) \Delta t+o(\Delta t)+v\left(X_{\tau+\Delta t}, K_{\tau+\Delta t}, \tau+\Delta t\right)$

Because such an action is not necessarily optimal, we must have

$$
e^{-\rho \tau} u\left(c_{\tau}\right) \Delta t+o(\Delta t)+v\left(X_{\tau+\Delta t}, K_{\tau+\Delta t}, \tau+\Delta t\right) \leq v(\xi, \eta, \tau)
$$

with equality holding if $t \rightarrow\left(K_{1}(t), x(t), c(t)\right), t \geq \tau$, is optimal during the infinitesimal time interval $[\tau, \tau+\Delta t)$.

Let us rewrite (1.A.1) as

$$
e^{-\rho \tau} u\left(c_{\tau}\right) \Delta t+o(\Delta l)+v\left(X_{\tau+\Delta t}, K_{\tau+\Delta t}, \tau+\Delta t\right)-v(\xi, \eta, \tau) \leq 0
$$

with equality holding if $t \rightarrow\left(x_{t}, c_{t}\right), t \geq \tau$, is optimal during the infinitesimal time interval $[\tau, \tau+\Delta t)$.

Now dividing (1.A.2) by $\Delta t$, and then letting $\Delta t \rightarrow 0$, we obtain

$$
e^{-\rho \tau} u\left(c_{\tau}\right)-D_{1} v(\xi, \eta, \tau) x_{\tau}+D_{2} v(\xi, \eta, \tau)\left(A \eta^{\alpha} x_{\tau}^{1-\alpha}-c_{\tau}\right)+D_{3} v(\xi, \eta, \tau) \leq 0
$$

with equality holding if $t \rightarrow\left(x_{t}, c_{t}\right), t \geq \tau$, is optimal at time $\tau$. Here $D_{1} v(\xi, \eta, \tau)$, $D_{2} v(\xi, \eta, \tau)$, and $D_{3} v(\xi, \eta, \tau)$ denote, respectively, the partial derivatives of $v(\xi, \eta, \tau)$ with respect to $\xi, \eta$, and $\tau$.

I represent this result more compactly under the following form, known as the Hamilton-Jacobi-Bellman equation for the model of exhaustible resources, technology 
substitution, and endogenous growth:

$$
0=\max _{(x, c)}\left\{e^{-\rho \tau}(c)-D_{1} v(\xi, \eta, \tau) x+D_{2} v(\xi, \eta, \tau)\left(A K^{\alpha} x^{1-\alpha}-c\right)+D_{3} v(\xi, \eta, \tau)\right\}
$$

and the optimal controls are the values of $x$ and $c$ that solves the maximization problem in (1.A.4).

Setting $(\xi, \eta, \tau)=(X, K, t)$, with $(X, K, t)$ being an arbitrary state of the system, in (1.A.4), I obtain the following general version of the Hamilton-Jacobi-Bellman equation that the optimal value must satisfy when at some arbitrary time $\ell$, the remaining stock of oil is $X$ and the stock of capital is $K$.

$$
0=\max _{(x, c)}\left\{e^{-\rho t}(c)-D_{1} v(X, K, t) x+D_{2} v(X, K, t)\left(A K^{\alpha} x^{1-\alpha}-c\right)+D_{3} v(X, K, t)\right\}
$$

and the values of $x$ and $c$ that solve the maximization problem in (1.A.5) constitute the optimal oil extraction rate and the optimal consumption when the system is in state $(X, K, t)$.

\section{Appendix 1.B The derivation of the Bellman func- tion}

The following listing provides the Mathematica software code for the symbolic computation of the Bellman equation, first in the case where $K>0$ and $X=0$, then for the case where $K>0$ and $X>0$. It also provides the code used to characterize under both scenarios the time paths of the extraction rate and consumption, as well as the time when the latter reaches its peak. 
We comment on some of the commands used for solving the Bellman function

The Bellman function when $K>0$ and $X=0$

ClearAl1 [u, HJB, $K, Y, X, X, f o c, s, v$, de, ede]

$u=\frac{c^{1-\theta}}{1-\theta} \quad$ (* here we define the utility function*)

$\frac{c^{1-\theta}}{1-\theta}$

HJB $=u-v \cdot[K] c-\rho v[K]$ (* this define the HJB equation *)

$\frac{\mathrm{C}^{2-\theta}}{1-\theta}-\rho \mathrm{V}[\mathrm{K}]-\mathrm{Cv}^{\prime}[\mathrm{K}]$

foc $[1]=D[H J B, c]$

(*we calculate the first order condition with respect to consumption

*)

$\mathrm{C}^{-\theta}-\mathrm{V}^{\prime}[\mathrm{K}]$

$s[1]=$ Solve[foc[1] = $0, c] / /$ Flatten(twe solve for the first order condition

*)

$\left\{\mathrm{C} \rightarrow \mathrm{v}^{\prime}[K]^{-1 / \theta}\right\}$

HJB $=$ HJB / . a [1] // PowerExpand // Fullsimplify

(*we substitute for the value of $c$ in the HJB equation *)

$-\rho \mathrm{v}[\mathrm{K}]+\frac{\theta \mathrm{v}^{\prime}[\mathrm{K}]^{1-\frac{1}{9}}}{1-\theta}$

$\mathbf{s}[2]=$ Solve $[\mathrm{HJB}=0, \mathrm{v} \cdot[\mathrm{K}]] / /$ Flatten

(* we solve for the derivative with respect to $k$ of the value function *)

$\left\{\mathrm{V}^{\prime}[\mathrm{K}] \rightarrow\left(\frac{(1-\theta) \rho \mathrm{V}[\mathrm{K}]}{\theta}\right)^{\frac{0}{1+\theta}}\right\}$

de $[1]=v^{\prime}[K]=\left(v^{\prime}[K] / . g[2]\right) / /$ PowerExpand

(*substitute in the the HJB equation to get the differential equation: *)

$V^{\prime}[K]=(1-\theta)^{\frac{\theta}{1 \cdot \theta}} \theta^{-\frac{\theta}{1+\theta}} \rho^{-\frac{\theta}{1 \cdot \theta}} \mathrm{V}[K]^{\frac{\theta}{1 \cdot \theta}}$

sde [1] = DSolve[de[1], $v, R] / /$ Flatten (* now we solve the obtained oDE of $v(R)$ *)

$\left\{v \rightarrow\right.$ Function $\left.\left[\{K\},\left(-\left((-1+\theta) \theta^{\frac{\theta}{1 \cdot \theta}}\right) /\left(K(1-\theta)^{\frac{\theta}{1-\theta}} \rho^{\frac{\theta}{1 \cdot \theta}}+\theta^{\frac{\theta}{1-\theta}} \mathrm{C}[1]\right)\right)^{-1+\theta}\right]\right\}$

$\operatorname{sde}[1]=\left\{v \rightarrow \operatorname{Function}\left[\{K\},\left(\left((1-\theta) \theta^{\frac{\theta}{-1+\theta}}\right) /\left(K(1-\theta)^{\frac{\theta}{-1+\theta}} \rho^{\frac{\theta}{-1+\theta}}+\theta^{-1+\theta} \mathrm{C}[1]\right)\right)^{-1+\theta}\right]\right\} / /$

PowerExpand ( $\star$ simplify *)

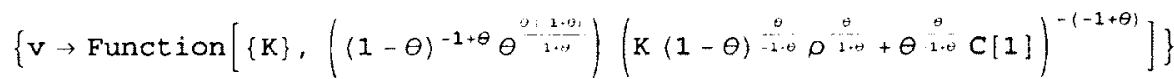

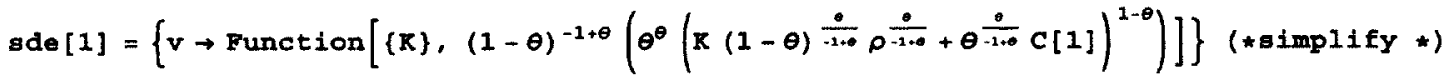

$\left\{\mathrm{V} \rightarrow \operatorname{Function}\left[\{\mathrm{K}\},(1-\theta)^{-1+\theta}\left(\theta^{\theta}\left(\mathrm{K}(1-\theta)^{\frac{\theta}{1-\theta}} \rho^{\frac{\theta}{1 \cdot \theta}}+\theta^{\frac{\theta}{1 \cdot \theta}} \mathrm{C}[1]\right)^{1-\theta}\right)\right]\right\}$

$v[0] /$. sde $[1](* g e t$ the $v(0) \star)$

$(1-\theta)^{-1+\theta} \theta^{\theta}\left(\theta^{\frac{\theta}{1+\alpha}} C[1]\right)^{1-\theta}$ 


$$
\begin{aligned}
& \text { sde }[1]=\left\{v \rightarrow \operatorname{Punction}\left[(K\},(1-\theta)^{-1+\theta}\left(\theta^{\theta}\left(K(1-\theta) \frac{\dot{-1+\theta}}{\rho^{-1+\theta}}\right)^{1-\theta}\right)\right]\right\} / / \\
& \text { PowerExpand (*substitute in the HJB equation *) } \\
& \left\{\mathrm{v} \rightarrow \text { Function }\left[(K\},(1-\theta)^{-1+\theta}\left(\theta^{\theta}\left(K^{1-\theta}\left((1-\theta)^{\frac{\theta \cdot 2 \cdot y}{1 \cdot \theta} \rho} \rho^{\frac{\theta \cdot 1 \cdot \theta}{1 \cdot \theta}}\right)\right)\right)\right]\right\} \\
& \text { sde }[1]=\left\{v \rightarrow \text { Function }\left[\{\mathrm{K}\}, \theta^{\theta}(1-\theta)^{-1} \rho^{-\theta} \mathrm{K}^{1-\theta}\right]\right\} / / \text { Fullsimplify } \\
& \left\{v \rightarrow \text { Function }\left[\{\mathbf{K}\}, \frac{\theta^{\Theta} \rho^{-\theta} \mathrm{K}^{1-\theta}}{1-\theta}\right]\right\} \\
& a=\frac{\theta^{\theta} \rho^{-\theta}}{1-\theta} \text { (*define a parameter that simplify the expression *) } \\
& \frac{\Theta^{\Theta} \rho^{-\theta}}{1-\theta} \\
& \text { ClearAl] [u, HJB, foc, } s, x, v, d e, s d e]
\end{aligned}
$$

- The Bellman function when there are both fossil fuels and capital

$$
\begin{aligned}
& a=\frac{\theta^{\theta} \rho^{-\theta}}{1-\theta}(\star \text { defined parameter *) } \\
& \frac{\theta^{\theta} \rho^{-\theta}}{1-\theta} \\
& u=\frac{c^{1-\theta}}{1-\theta}(\star \text { the utility function } \star) \\
& \frac{c^{1-\theta}}{1-\theta} \\
& \mathbf{Y}=A K^{\alpha} x^{1-\alpha}(\star \text { define the production function } \star) \\
& A K^{a} x^{1-a} \\
& k=Y-c(* \text { define the equation of motion//the budget constraint } *) \\
& -C+A K^{\alpha} x^{1-\alpha} \\
& v=K^{1-\theta} \omega\left[\frac{X}{K}\right] \\
& \text { (*this is the form of the value function obtained from the analytical solution *) } \\
& \mathrm{K}^{1-\theta} \omega\left[\frac{\mathrm{X}}{\mathrm{K}}\right] \\
& d[1]=D[v, X](\star t a k e \text { the derivative of } v \text { with respect to } x \star) \\
& \mathrm{K}^{-\theta} \omega^{\prime}\left[\frac{\mathrm{X}}{\mathrm{K}}\right] \\
& d[2]=D[v, K](\star \text { take the derivative of } v \text { with respect to } K *) \\
& \mathrm{K}^{-\theta}(1-\theta) \omega\left[\frac{\mathrm{X}}{\mathrm{K}}\right]-\mathrm{K}^{-1-\theta} \mathrm{X} \omega^{\circ}\left[\frac{\mathrm{X}}{\mathrm{K}}\right] \\
& \text { HJB }=u-d[1] x+d[2] k-\rho v(* u s e \text { the derivative of } v \text { with respect to } x \text { and } x \text { in the } \mathrm{EJB} * \text { ) } \\
& \frac{\mathrm{C}^{1-\theta}}{1-\theta}-\mathrm{K}^{1-\theta} \rho \omega\left[\frac{\mathrm{X}}{\mathrm{K}}\right]-\mathrm{K}^{-\theta} \mathrm{x} \omega^{\prime}\left[\frac{\mathrm{X}}{\mathrm{K}}\right]+\left(-\mathrm{C}+\mathrm{A} \mathrm{K}^{\alpha} \mathrm{X}^{1-\alpha}\right)\left(\mathrm{K}^{-\theta}(1-\theta) \omega\left[\frac{\mathrm{X}}{\mathrm{K}}\right]-\mathrm{K}^{-1-\theta} \mathrm{X} \omega^{\prime}\left[\frac{\mathrm{X}}{\mathrm{K}}\right]\right)
\end{aligned}
$$


$\mathrm{HJB}=\mathrm{HJB} / . \mathrm{X} \rightarrow \lambda \mathrm{K}($ agsign to $\mathrm{X}$ the expression $\lambda \mathrm{K} \star)$

$\frac{\mathrm{C}^{1-\theta}}{1-\theta}-\mathrm{K}^{1-\theta} \rho \omega[\lambda]-\mathrm{K}^{-\theta} \times \omega^{\prime}[\lambda]+\left(-\mathbf{C}+\mathbf{A} \mathrm{K}^{\alpha} \mathrm{x}^{1-\alpha}\right)\left(\mathrm{K}^{-\theta}(1-\theta) \omega[\lambda]-\mathrm{K}^{-\theta} \lambda \omega^{\prime}[\lambda]\right)$

foc $[1]=D[H J B, c]$ (*take the first derivative of HJB with respect to $c$ *)

$\mathrm{C}^{-\theta}-\mathrm{K}^{-\theta}(1-\theta) \omega[\lambda]+\mathrm{K}^{-\theta} \lambda \omega^{\prime}[\lambda]$

$\Delta[1]=$ Solve[foc $[1]=0, c] / /$ Flatten $(*$ solve the foc with respect to $x *)$

$\left\{c \rightarrow\left(K^{-\theta} \omega[\lambda]-K^{-\theta} \theta \omega[\lambda]-K^{-\theta} \lambda \omega^{\prime}[\lambda]\right)^{-1 / \theta}\right\}$

$c=c / .8[1](\star A s s i g n$ to $c$ the solution in $B[1] *)$

$\left(K^{-\theta} \omega[\lambda]-K^{-\theta} \theta \omega[\lambda]-K^{-\theta} \lambda \omega^{\prime}[\lambda]\right)^{-1 / \theta}$

$c=c / /$ Fullsimplify

$\left(K^{-\theta}\left(\omega[\lambda]-\theta \omega[\lambda]-\lambda \omega^{\prime}[\lambda]\right)\right)^{-1 / \theta}$

$c=c / /$ Powerkxpand

$K\left(\omega[\lambda]-\theta \omega[\lambda]-\lambda \omega^{\prime}[\lambda]\right)^{-1 / \theta}$

HJB

$-K^{1-\theta} \rho \omega[\lambda]-K^{-\theta} \times \omega^{\prime}[\lambda]+\frac{1}{1-\theta}\left(K\left(\omega[\lambda]-\theta \omega[\lambda]-\lambda \omega^{\prime}[\lambda]\right)^{-1 \theta}\right)^{1-\theta}+$

$\left(\mathrm{K}^{-\theta}(1-\theta) \omega[\lambda]-\mathrm{K}^{-\theta} \lambda \omega^{\prime}[\lambda]\right)\left(\mathrm{A} \mathrm{K}^{\alpha} \mathrm{x}^{1-\alpha}-\mathrm{K}\left(\omega[\lambda]-\theta \omega[\lambda]-\lambda \omega^{\prime}[\lambda]\right)^{-1 / \theta}\right)$

foc $[2]=D[H J B, x]$

$-K^{-\theta} \omega^{\prime}[\lambda]+A K^{\alpha} x^{-\alpha}(1-\alpha)\left(K^{-\theta}(1-\theta) \omega[\lambda]-K^{-\theta} \lambda \omega^{\prime}[\lambda]\right)$

$\boldsymbol{s}[2]=$ Solve[foc $[2]=0, \mathbf{x}] / /$ Flatten

$\left\{x \rightarrow\left(\frac{1}{\omega^{\prime}[\lambda]}\right.\right.$

$\left.\left.\mathrm{K}^{\theta}\left(\mathrm{AK} \mathrm{K}^{\alpha-\theta} \omega[\lambda]-\mathbf{A} \mathrm{K}^{\alpha-\theta} \alpha \omega[\lambda]-\mathbf{A} \mathrm{K}^{\alpha-\theta} \theta \omega[\lambda]+\mathbf{A} \mathrm{K}^{\alpha-\theta} \alpha \theta \omega[\lambda]-\mathbf{A} \mathrm{K}^{\alpha-\theta} \lambda \omega^{\prime}[\lambda]+\mathbf{A} \mathrm{K}^{\alpha-\theta} \alpha \lambda \omega^{\prime}[\lambda]\right)\right)^{\frac{1}{\alpha}}\right\}$

$\mathbf{x}=\mathbf{x} / . \mathbf{s}[2]$

$\left(\frac{1}{\omega^{\prime}[\lambda]} K^{\theta}\left(A K^{\alpha-\theta} \omega[\lambda]-A K^{\alpha-\theta} \alpha \omega[\lambda]-A K^{\alpha-\theta} \theta \omega[\lambda]+A K^{\alpha-\theta} \alpha \theta \omega[\lambda]-A K^{\alpha-\theta} \lambda \omega^{\prime}[\lambda]+A K^{\alpha-\theta} \alpha \lambda \omega^{\prime}[\lambda]\right)\right)^{\frac{1}{a}}$

x / / Pullsimplify

$\left(\frac{1}{\omega^{\prime}[\lambda]} A K^{\alpha}(-1+\alpha)\left((-1+\theta) \omega[\lambda]+\lambda \omega^{\prime}[\lambda]\right)\right)^{\frac{1}{\alpha}}$

$\mathbf{x}=\left(\left(\mathbf{A} \mathrm{K}^{\alpha}(1-\alpha)\left((1-\theta) \omega[\lambda]-\lambda \omega^{\prime}[\lambda]\right)\right) / \omega^{\prime}[\lambda]\right)^{\frac{1}{a}}$

$\left(\frac{1}{\omega^{\prime}[\lambda]} \mathrm{AK}^{\alpha}(1-\alpha)\left((1-\theta) \omega[\lambda]-\lambda \omega^{\prime}[\lambda]\right)\right)^{\frac{1}{a}}$

$\mathbf{x}=\mathbf{x} / /$ PowerExpand

$\mathrm{A}^{\frac{1}{x}} \mathrm{~K}(1-\alpha)^{\frac{1}{3}} \omega^{\prime}[\lambda]^{-1 / \alpha}\left((1-\theta) \omega[\lambda]-\lambda \omega^{\prime}[\lambda]\right)^{\frac{1}{x}}$ 


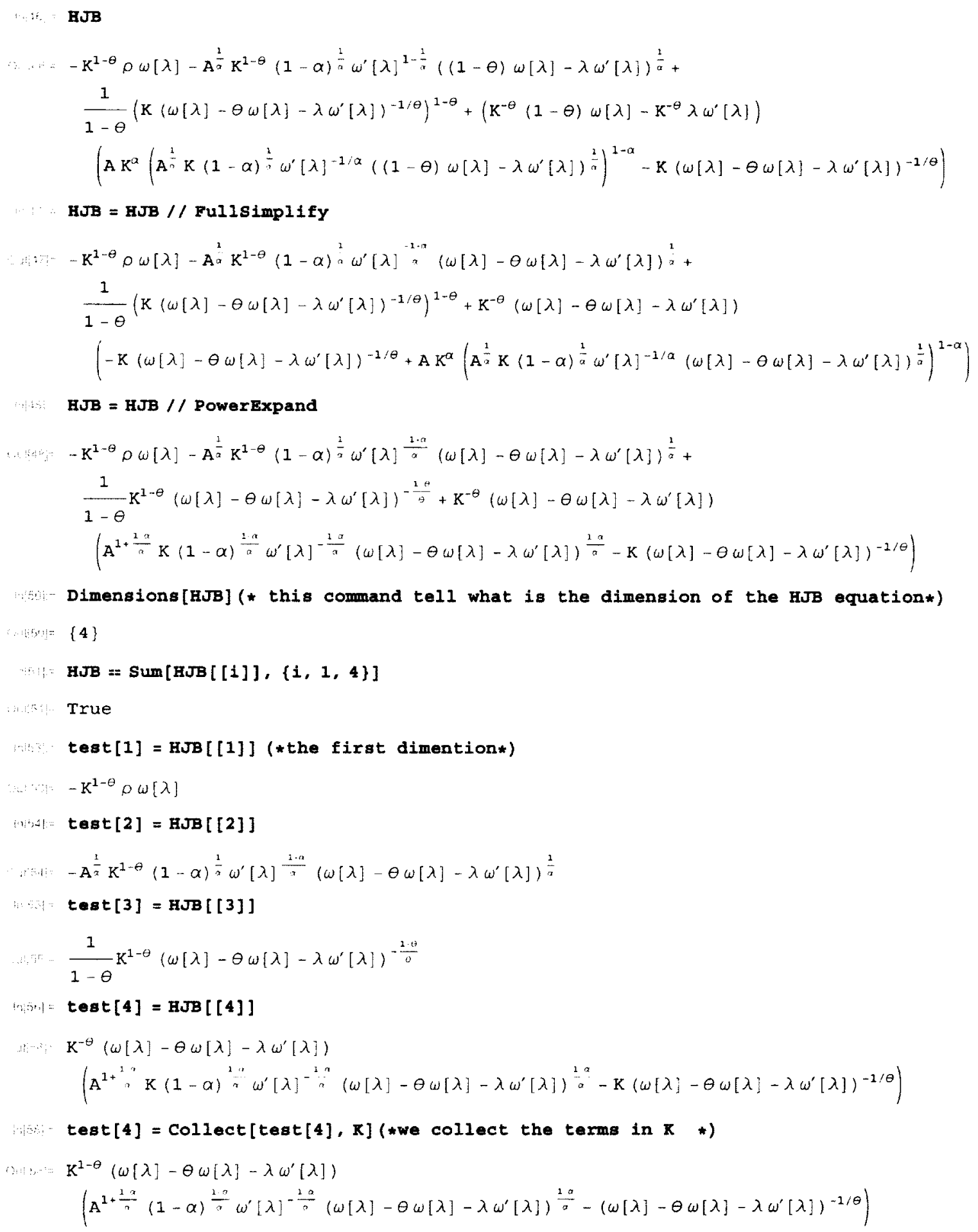


HJB $=\operatorname{sum}[\operatorname{test}[i],\{i, 1,4\}]$

$$
\begin{aligned}
& -K^{1-\theta} \rho \omega[\lambda]-A^{\frac{1}{a}} K^{1-\theta}(1-\alpha)^{\frac{1}{a}} \omega^{\prime}[\lambda] \frac{1 \cdot \alpha}{a}\left(\omega[\lambda]-\theta \omega[\lambda]-\lambda \omega^{\prime}[\lambda]\right)^{\frac{1}{\alpha}}+ \\
& \frac{1}{1-\theta} \mathrm{K}^{1-\theta}\left(\omega[\lambda]-\theta \omega[\lambda]-\lambda \omega^{\prime}[\lambda]\right)^{-\frac{2 \theta}{\theta}}+\mathrm{K}^{1-\theta}\left(\omega[\lambda]-\theta \omega[\lambda]-\lambda \omega^{\prime}[\lambda]\right) \\
& \left(A^{1+\frac{1 \alpha}{0}}(1-\alpha)^{1 \omega} \omega^{\prime}[\lambda]^{-\frac{1 \cdot x}{2}}\left(\omega[\lambda]-\theta \omega[\lambda]-\lambda \omega^{\prime}[\lambda]\right)^{\frac{10}{a}}-\left(\omega[\lambda]-\theta \omega[\lambda]-\lambda \omega^{\prime}[\lambda]\right)^{-1 / \theta}\right)
\end{aligned}
$$

$\mathrm{HJB}=\operatorname{Collect}\left[\mathrm{HJB}, \mathbf{R}^{1-\theta}\right]$

$\mathrm{K}^{1-\theta}\left(-\rho \omega[\lambda]-\mathrm{A}^{\frac{1}{2}}(1-\alpha)^{\frac{1}{\alpha}} \omega^{\prime}[\lambda]^{\frac{1 \cdot a}{x}}\left(\omega[\lambda]-\theta \omega[\lambda]-\lambda \omega^{\prime}[\lambda]\right)^{\frac{1}{a}-}\right.$

$$
\begin{aligned}
& A^{1+\frac{1 a}{a}}(1-\alpha)^{\frac{1 a}{a}} \lambda \omega^{\prime}[\lambda]^{1+\frac{1 \cdot a}{a}}\left(\omega[\lambda]-\theta \omega[\lambda]-\lambda \omega^{\prime}[\lambda]\right)^{\frac{1 a}{a}}+A^{1+\frac{1 a}{a}}(1-\alpha)^{\frac{1 \cdot a}{a}} \omega[\lambda] \omega^{\prime}[\lambda]^{\frac{1 \cdot a}{a}} \\
& \left(\omega[\lambda]-\theta \omega[\lambda]-\lambda \omega^{\prime}[\lambda]\right)^{\frac{1 a}{a}}-\mathrm{A}^{1+\frac{1+a}{a}}(1-\alpha)^{\frac{1 a}{a}} \theta \omega[\lambda] \omega^{\prime}[\lambda]^{\frac{1 \cdot \alpha}{a}}\left(\omega[\lambda]-\theta \omega[\lambda]-\lambda \omega^{\prime}[\lambda]\right)^{\frac{1 a}{a}}- \\
& \omega[\lambda]\left(\omega[\lambda]-\theta \omega[\lambda]-\lambda \omega^{\prime}[\lambda]\right)^{-1 / \theta}+\theta \omega[\lambda]\left(\omega[\lambda]-\theta \omega[\lambda]-\lambda \omega^{\prime}[\lambda]\right)^{-1 / \theta}+ \\
& \left.\lambda \omega^{\prime}[\lambda]\left(\omega[\lambda]-\theta \omega[\lambda]-\lambda \omega^{\prime}[\lambda]\right)^{-1 / \theta}+\frac{1}{1-\theta}\left(\omega[\lambda]-\theta \omega[\lambda]-\lambda \omega^{\prime}[\lambda]\right)^{-\frac{1 \cdot \theta}{\theta}}\right)
\end{aligned}
$$

\section{HJB $=$ HJB $/ /$ Fullsimplify}

$$
\begin{aligned}
& \left(\mathrm{K}^{1-\theta} \omega^{\prime}[\lambda]^{-1 / \alpha}\left(\omega[\lambda]-\theta \omega[\lambda]-\lambda \omega^{\prime}[\lambda]\right)^{-1 / \theta}\right. \\
& \quad\left((-1+\alpha) \theta \lambda \omega^{\prime}[\lambda]^{1+{ }^{2}}-\mathrm{A}^{2} \alpha(1-\alpha)^{2} \alpha(-1+\theta) \omega^{\prime}[\lambda]\left(\omega[\lambda]-\theta \omega[\lambda]-\lambda \omega^{\prime}[\lambda]\right)^{\frac{1}{\alpha}+\frac{2}{\theta}}+\right. \\
& \left.\left.\quad(-1+\alpha)(-1+\theta) \omega[\lambda] \omega^{\prime}[\lambda]^{1} \cdot\left(\theta-\rho\left(\omega[\lambda]-\theta \omega[\lambda]-\lambda \omega^{\prime}[\lambda]\right)^{\frac{1}{\theta}}\right)\right)\right) /((-1+\alpha)(-1+\theta))
\end{aligned}
$$

(M) Dimensions [HJB]

$\{6\}$

нЛВ $==\prod_{1=1}^{6}$ нЈB [ [i] ]

True

HJB [ [1] ]

$\mathrm{K}^{1-\theta}$

HSB [ [ 2 ] ]

$\frac{1}{-1+\alpha}$

HJB [ [3] ]

$\frac{1}{-1+\theta}$

HJB [ [4] ]

$\omega^{\prime}[\lambda]^{-1 / \alpha}$

HJB [ [5] ]

$\left(\omega[\lambda]-\theta \omega[\lambda]-\lambda \omega^{\prime}[\lambda]\right)^{-1 / \theta}$

HJB [ [6] ]

$(-1+\alpha) \theta \lambda \omega^{\prime}[\lambda]^{1+\frac{1}{\alpha}}-\mathrm{A}^{\frac{1}{\alpha}}(1-\alpha)^{\frac{2}{\alpha}} \alpha(-1+\theta) \omega^{\prime}[\lambda]\left(\omega[\lambda]-\theta \omega[\lambda]-\lambda \omega^{\prime}[\lambda]\right)^{\frac{1}{\alpha}+\frac{1}{\alpha}}+$

$(-1+\alpha)(-1+\theta) \omega[\lambda] \omega^{\prime}[\lambda]^{\frac{1}{a}}\left(\theta-\rho\left(\omega[\lambda]-\theta \omega[\lambda]-\lambda \omega^{\prime}[\lambda]\right)^{\frac{1}{\theta}}\right)$ 


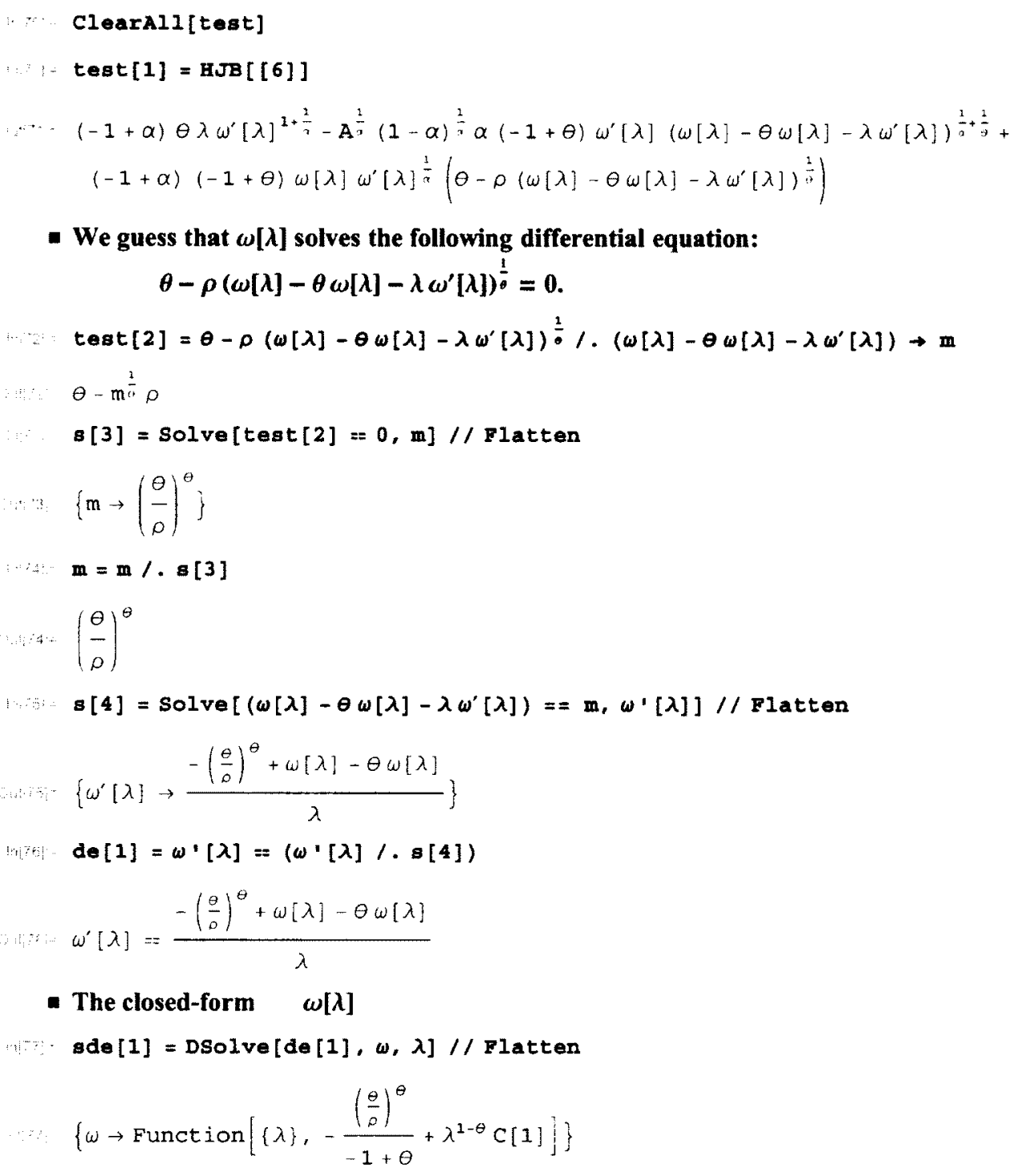

- We guess that $\omega[\lambda]$ solves the following differential equation:

$$
\theta-\rho\left(\omega[\lambda]-\theta \omega[\lambda]-\lambda \omega^{\prime}[\lambda]^{\frac{1}{\theta}}=0 .\right.
$$

- The closed-form $\omega[\lambda]$ sde $[1]=$ DSolve $[$ de $[1], \omega, \lambda] / /$ Flatten $\left\{\omega \rightarrow\right.$ Function $\left.\left[\{\lambda\},-\frac{\left(\frac{\theta}{\rho}\right)^{\theta}}{-1+\theta}+\lambda^{1-\theta} \mathrm{C}[1]\right\}\right\}$

- Verifying that $\omega[\lambda]$ satisfies the initial condition $(\omega[0] /$. ade $[1]) / /$ PowerExpand

$-\frac{\theta^{\theta} p^{-\theta}}{-1+\theta}+0^{1-\theta} \mathrm{C}[1]$

* - // Pullsimplify

$0^{1-\theta} C[1]$ 


\section{- Determining the constant of integration $C[1]$}

test $[3]=$ test $[1] /$ sde $[1]$

$(-1+\alpha) \theta \lambda\left((1-\theta) \lambda^{-\theta} C[1]\right)^{1+\frac{1}{3}}-A^{\frac{1}{2}}(1-\alpha)^{\frac{1}{2}} \alpha(1-\theta)(-1+\theta)$

$$
\lambda^{-\theta} \mathrm{C}[1]\left(-\frac{\left(\frac{\theta}{\rho}\right)^{\theta}}{-1+\theta}+\lambda^{1-\theta} \mathrm{C}[1]-(1-\theta) \lambda^{1-\theta} \mathrm{C}[1]-\theta\left(-\frac{\left(\frac{\theta}{\rho}\right)^{\theta}}{-1+\theta}+\lambda^{1-\theta} \mathrm{C}[1]\right)\right)^{\frac{2}{\alpha}+\frac{1}{\theta}}+
$$

$(-1+\alpha)(-1+\theta)\left((1-\theta) \lambda^{-\theta} C[1]\right)^{\frac{2}{\alpha}}\left(-\frac{\left(\frac{\theta}{\theta}\right)^{\theta}}{-1+\theta}+\lambda^{1-\theta} C[1]\right)$

$$
\left(\theta-\rho\left(-\frac{\left(\frac{\theta}{\theta}\right)^{\theta}}{-1+\theta}+\lambda^{1-\theta} \mathrm{C}[1]-(1-\theta) \lambda^{1-\theta} \mathrm{C}[1]-\theta\left(-\frac{\left(\frac{\theta}{\rho}\right)^{\theta}}{-1+\theta}+\lambda^{1-\theta} \mathrm{C}[1]\right)\right)^{\frac{2}{\theta}}\right)
$$

test [3] = test [3] // Fullsimplify

$\lambda^{-\theta}\left(\mathrm{A}^{\frac{2}{a}}(1-\alpha)^{\frac{1}{2}} \alpha(-1+\theta)^{2}\left(\left(\frac{\theta}{\rho}\right)^{\theta}\right)^{\frac{1}{a}+\frac{1}{\beta}} \mathrm{C}[1]-\right.$

$$
\left.(-1+\alpha)\left(-(-1+\theta) \lambda^{-\theta} \mathrm{C}[1]\right)^{\frac{1}{\alpha}}\left(\lambda^{\theta}\left(\frac{\theta}{\rho}\right)^{\theta}\left(\theta-\left(\left(\frac{\theta}{\rho}\right)^{\theta}\right)^{\frac{1}{\theta}} \rho\right)+(-1+\theta) \lambda\left(\left(\frac{\theta}{\rho}\right)^{\theta}\right)^{\frac{1}{\beta}} \rho \mathrm{C}[1]\right)\right)
$$

$\operatorname{test}[3]=\operatorname{test}[3] / /$ PowerExpand

$\lambda^{-\theta}\left(A^{\frac{1}{a}}(1-\alpha)^{\frac{1}{a}} \alpha(-1+\theta)^{2} \theta^{\left(\frac{1}{\alpha}+\frac{1}{a}\right) \theta} \rho^{-\left(\frac{1}{a}+\frac{1}{\theta}\right) \theta} \mathrm{C}[1]-(-1)^{\frac{2}{\alpha}}(-1+\alpha)(-1+\theta)^{1+\frac{1}{a}} \theta \lambda^{1-\frac{4}{\alpha}} \mathrm{C}[1]^{1+\frac{1}{\alpha}}\right)$

test $[3] / . C[1] \rightarrow b$

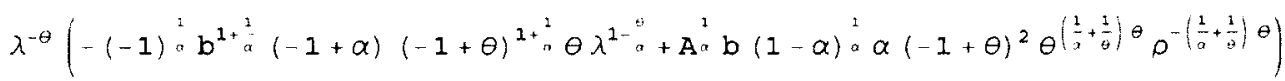

- Setting $\alpha=\theta$

test $[4]=\operatorname{test}[3] /, \alpha \rightarrow \theta$

$\lambda^{-\theta}\left(\frac{1}{\rho^{2}} A^{\frac{1}{\partial}}(1-\theta)^{\frac{1}{3}}(-1+\theta)^{2} \theta^{3} C[1]-(-1)^{\frac{1}{\theta}}(-1+\theta)^{2+\frac{1}{\theta}} \theta C[1]^{2+\frac{1}{\theta}}\right)$

$s[5]=$ Solve $[$ test $[4]=0, C[1]] / /$ glatten

$\left\{C[1] \rightarrow 0, C[1] \rightarrow\left(\frac{1}{\theta^{2}}(-1)^{\frac{1}{\theta}} A^{-1 / \theta}(1-\theta)^{-1 / \theta}(-1+\theta)^{\frac{1}{\theta}} \rho^{2}\right)^{-\theta}\right\}$

$s[5]=s[5] / /$ PowerExpand

$\therefore \quad\left\{C[1] \rightarrow 0, C[1] \rightarrow-\frac{A(1-\theta) \theta^{2 \theta} p^{-2 \theta}}{-1+\theta}\right\}$ 
- Accept the value $C[1]=-\frac{A(1-\theta) \theta^{2 \theta} \rho^{-2 \theta}}{-1+\theta}$. The closed-form expression $\quad \omega[\lambda]$ issde= sde [1]

$\left\{\omega \rightarrow\right.$ Function $\left.\left[\{\lambda],-\frac{\left(\frac{\theta}{0}\right)^{\theta}}{-1+\theta}+\lambda^{1-\theta} \mathrm{C}[1]\right]\right\}$

$\operatorname{sde}[1]=\operatorname{sde}[1] / . s[5][[2]]$

$\left\{\omega \rightarrow\right.$ Function $\left.\left[\{\lambda\},-\frac{\left(\frac{\theta}{b}\right)^{\theta}}{-1+\theta}+\left(\lambda^{1-\theta}\left(-A(1-\theta) \theta^{2 \theta} \rho^{-2 \theta}\right)\right) /(-1+\theta)\right]\right\}$

sde $[1]=\left\{\omega \rightarrow \operatorname{Function}\left[\{\lambda\}, \frac{\left(\frac{\theta}{\rho}\right)^{\theta}}{1-\theta}+A\left(\frac{\theta}{\rho}\right)^{2 \theta} \lambda^{1-\theta}\right]\right\}$

$\left\{\omega \rightarrow\right.$ Function $\left.\left[\{\lambda\}, \frac{\left(\frac{\theta}{\rho}\right)^{\theta}}{1-\theta}+A\left(\frac{\theta}{\rho}\right)^{2 \theta} \lambda^{1-\theta}\right]\right\}$

- The optimal consumption

c

$\mathrm{K}(\omega[\lambda]-\theta \omega[\lambda]-\lambda \omega[\lambda])^{-1 / \theta}$

$c=c / . \lambda \rightarrow \frac{x}{x}$

$\mathrm{K}\left(\omega\left[\frac{\mathrm{X}}{\mathrm{K}}\right]-\theta \omega\left[\frac{\mathrm{X}}{\mathrm{K}}\right]-\frac{\mathrm{X} \omega^{*}\left[\frac{\mathrm{x}}{\mathrm{K}}\right]}{\mathrm{K}}\right)^{-1 / \theta}$

$c=c / . \operatorname{sde}[1]$

$\mathrm{K}\left(-\theta\left(\frac{\left(\frac{\theta}{\rho}\right)^{\theta}}{I-\theta}+\mathrm{A}\left(\frac{\mathrm{X}}{\mathrm{K}}\right)^{1-\theta}\left(\frac{\theta}{\rho}\right)^{2 \theta}\right)+\frac{\left(\frac{\theta}{\rho}\right)^{\theta}}{1-\theta}+\mathrm{A}\left(\frac{\mathrm{X}}{\mathrm{K}}\right)^{1-\theta}\left(\frac{\theta}{\rho}\right)^{2 \theta}-\frac{1}{\mathrm{~K}} \mathrm{AX}\left(\frac{\mathrm{X}}{\mathrm{K}}\right)^{-\theta}(1-\theta)\left(\frac{\theta}{\rho}\right)^{2 \theta}\right)^{-1 / \theta}$

$c=c / /$ PowerExpand

$\mathrm{K}\left(\mathrm{AK}^{-1+\theta} \mathrm{X}^{1-\theta} \theta^{2 \theta} \rho^{-2 \theta}-\mathrm{AK}^{-1+\theta} \mathrm{X}^{1-\theta}(1-\theta) \theta^{2 \theta} \rho^{-2 \theta}+\frac{\theta^{\theta} \rho^{-\theta}}{1-\theta}-\theta\left(\mathrm{AK} \mathrm{K}^{-1+\theta} \mathrm{X}^{1-\theta} \theta^{2 \theta} \rho^{-2 \theta}+\frac{\theta^{\theta} \rho^{-\theta}}{1-\theta}\right)\right)^{-1 / \theta}$

$c=c / /$ Pullsimplify

$\mathrm{K}\left(\theta^{\theta} \rho^{-\theta}\right)^{-1 / \theta}$

- Consumption is a constant proportion of the capital stock

int- $c=c / /$ PowerExpand

$\frac{\mathrm{K} \rho}{\theta}$

- Extraction rate is a constant proportion of the capital stock

$\mathbf{x}$

$\mathbb{A}^{\frac{1}{\alpha}} \mathrm{K}(1-\alpha)^{\frac{1}{\alpha}} \omega^{\prime}[\lambda]^{-1 / \alpha}\left((1-\theta) \omega[\lambda]-\lambda \omega^{\prime}[\lambda]^{\frac{1}{\alpha}}\right.$ 
$x=x / \lambda \rightarrow \frac{x}{x}$

$A^{\frac{1}{2}} \mathrm{~K}(1-\alpha) \stackrel{\frac{1}{*}}{\omega} \omega^{\prime}\left[\frac{\mathrm{X}}{\mathrm{K}}\right]^{-1 / \alpha}\left((1-\theta) \omega\left[\frac{\mathrm{X}}{\mathrm{K}}\right]-\frac{\mathrm{X} \omega^{\prime}\left[\frac{\mathrm{X}}{\mathrm{K}}\right]}{\mathrm{K}}\right)^{\frac{1}{2}}$

$x=x / . \mathbf{s d e}[1]$

$A^{\frac{1}{\gamma}} \mathrm{K}(1-\alpha)^{\frac{1}{\alpha}}\left((1-\theta)\left(\frac{\left(\frac{\theta}{\rho}\right)^{\theta}}{1-\theta}+\mathrm{A}\left(\frac{\mathrm{X}}{\mathrm{K}}\right)^{1-\theta}\left(\frac{\theta}{\rho}\right)^{2 \theta}\right)-\frac{1}{\mathrm{~K}} \mathrm{AX}\left(\frac{\mathrm{X}}{\mathrm{K}}\right)^{-\theta}(1-\theta)\left(\frac{\theta}{\rho}\right)^{2 \theta}\right)^{\frac{1}{\alpha}}\left(\mathrm{A}\left(\frac{\mathrm{X}}{\mathrm{K}}\right)^{-\theta}(1-\theta)\left(\frac{\theta}{\rho}\right)^{2 \theta}\right)^{-1 / \alpha}$

$x=x / . \alpha+\theta$

$A^{\frac{1}{2}} \mathrm{~K}(1-\theta)^{\frac{3}{3}}\left((1-\theta)\left(\frac{\left(\frac{\theta}{\rho}\right)^{\theta}}{1-\theta}+\mathrm{A}\left(\frac{\mathrm{X}}{\mathrm{K}}\right)^{1-\theta}\left(\frac{\theta}{\rho}\right)^{2 \theta}\right)-\frac{1}{\mathrm{~K}} \mathrm{AX}\left(\frac{\mathrm{X}}{\mathrm{K}}\right)^{-\theta}(1-\theta)\left(\frac{\theta}{\rho}\right)^{2 \theta}\right)^{\frac{1}{\theta}}\left(\mathrm{A}\left(\frac{\mathrm{X}}{\mathrm{K}}\right)^{-\theta}(1-\theta)\left(\frac{\theta}{\rho}\right)^{2 \theta}\right)^{-1 / \theta}$

$x=x / /$ PowerExpand

$\frac{1}{\theta^{2}} X^{2}\left(-\operatorname{AK}^{-1+\theta} \mathrm{X}^{1-\theta}(1-\theta) \theta^{2 \theta} \rho^{-2 \theta}+(1-\theta)\left(\mathrm{AK}^{-1+\theta} \mathrm{X}^{1-\theta} \theta^{2 \theta} \rho^{-2 \theta}+\frac{\theta^{\theta} \rho^{-\theta}}{1-\theta}\right)\right)^{\frac{1}{3}}$

$x=x / /$ Fullsimplify

$\frac{X \rho^{2}\left(\theta^{\theta} \rho^{-\theta}\right)^{\frac{2}{\theta}}}{\theta^{2}}$

$\mathbf{x}=\mathbf{x} / /$ PowerExpand

$\frac{\mathrm{X} \rho}{\theta}$

- Motion of the remaining stock of fossil fuels

$\mathbf{x}$

$\frac{\mathrm{x} \rho}{\theta}$

$d e[2]=x \cdot[t]=-(x / . x \rightarrow x[t])$

$\therefore+x^{\prime}[t]=-\frac{\rho X[t]}{\theta}$

sde $[2]=$ DSolve $[\{$ de $[2], x[0]=x 0\}, x, t] / /$ Flatten

$\left\{x \rightarrow \operatorname{Function}\left[\{t\}, e^{-\frac{t p}{*}} x_{0}\right]\right\}$

- Motion of the stock of capital

$$
\begin{aligned}
& x=-\frac{K \rho}{\theta}+\mathrm{AK}^{\alpha}\left(\frac{\mathrm{X} \rho}{\theta}\right)^{1-\alpha} \\
& -\frac{\mathrm{K} \rho}{\theta}+\mathrm{AK}^{\alpha}\left(\frac{\mathrm{X} \rho}{\theta}\right)^{1-\alpha}
\end{aligned}
$$


$-\frac{\mathrm{K} \rho}{\theta}+\mathrm{AK} \mathrm{K}^{\alpha}\left(\frac{\mathrm{X} \rho}{\theta}\right)^{1-\alpha}$

$k=k / /$ PowerExpand

$-\frac{\mathrm{K} \rho}{\theta}+\mathrm{A} \mathrm{K}^{\alpha} \mathrm{X}^{1-\alpha} \theta^{-1+\alpha} \rho^{1-\alpha}$

$\operatorname{de}[3]=x \cdot[t]=(k / .\{x \rightarrow(x[t] / . \operatorname{sde}[2]), x \rightarrow K[t]\})$

$K^{\prime}[t]=-\frac{\rho K[t]}{\theta}+A\left(e^{-\frac{t a}{*} \times 0}\right)^{1-\alpha} \theta^{-1+\alpha} \rho^{1-\alpha} K[t]^{\alpha}$

de[3] $=$ de[3] // PowerExpand

$K^{\prime}[t]=-\frac{\rho K[t]}{\theta}+A e^{-\frac{t 11 \alpha}{\theta}} \times 0^{1-\alpha} \theta^{-1+\alpha} \rho^{1-\alpha} K[t]^{\alpha}$

$\operatorname{de}[3]=\operatorname{de}[3] / \alpha \rightarrow \theta$

$\mathrm{K}^{\prime}[\mathrm{t}]=-\frac{\rho \mathrm{K}[\mathrm{t}]}{\theta}+\mathrm{A} \mathrm{e}^{-\frac{1,2 \theta: \theta}{\theta}} \times 0^{1-\theta} \theta^{-1+\theta} \rho^{1-\theta} \mathrm{K}[\mathrm{t}]^{\theta}$

sde $[3]=\operatorname{DSolve}[(\operatorname{de}[3], K[0]==K 0\}, K, t] / /$ Flatten

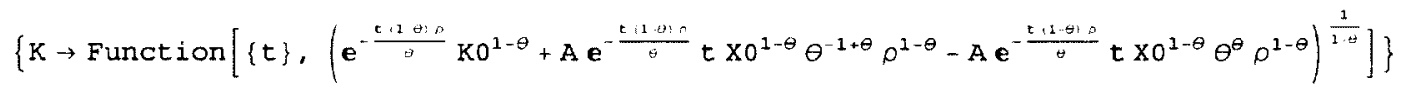

sde $[3] / .\left\{\mathrm{XO}_{0} \rightarrow \mathrm{x}_{0}, \mathrm{xO} \rightarrow \mathrm{K}_{0}\right\}$

$\left\{K \rightarrow\right.$ Function $\left.\left[\{t\},\left(e^{-\frac{t(1 \theta: n}{\theta}} K_{0}^{1-\theta}+A e^{-\frac{t(1-1)}{\theta}} t X_{0}^{1-\theta} \theta^{-1+\theta} \rho^{1-\theta}-A e^{-\frac{t i 1 \theta \rho}{\theta}} t X_{0}^{1-\theta} \theta^{\theta} \rho^{1-\theta}\right)^{\frac{1}{1 \theta}}\right]\right\}$

$R t=R[t] /$. sde $[3]$

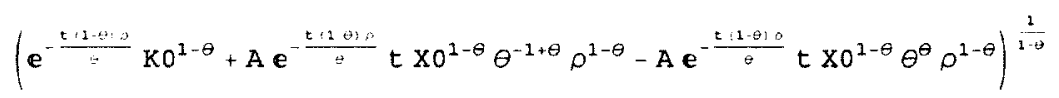

$K t=\left(\operatorname{Collect}\left[e^{-\frac{t(1-\theta) \rho}{\theta}} \times 0^{1-\theta}+A e^{-\frac{t(1-\theta) \rho}{\theta}} t \times 0^{1-\theta} \theta^{-1+\theta} \rho^{1-\theta}-A e^{-\frac{t(1-\theta) \rho}{\theta}} t \times 0^{1-\theta} \theta^{\theta} \rho^{1-\theta}, e^{-\frac{\varepsilon(2-\theta) \theta}{\theta}}\right]\right)^{\frac{1}{2-\theta}}$

$\left(\mathrm{e}^{-\frac{\mathrm{t} 11 \cdots n}{\theta}}\left(\mathrm{KO}^{1-\theta}+\mathrm{At} \times \mathrm{O}^{1-\theta} \theta^{-1+\theta} \mathrm{\rho}^{1-\theta}-\mathrm{At} \times \mathrm{O}^{1-\theta} \theta^{\theta} \mathrm{p}^{1-\theta}\right)\right)^{\frac{1}{1-\theta}}$

Kt // PowerExpand

$e^{-\frac{t}{\eta}}\left(\mathrm{KO}^{1-\theta}+\operatorname{Atx} \mathrm{O}^{1-\theta} \theta^{-1+\theta} \rho^{1-\theta}-\operatorname{Atx} 0^{1-\theta} \theta^{\theta} \rho^{1-\theta}\right)^{\frac{1}{1-\theta}}$

test [4] $=\log [K t] / /$ PowerExpand

$\frac{1}{1-\theta}\left(-\frac{\mathrm{t}(1-\theta) \rho}{\theta}+\log \left[\mathrm{K} 0^{1-\theta}+\mathrm{At} \times \mathrm{XO}^{1-\theta} \theta^{-1+\theta} \rho^{1-\theta}-\mathrm{At} \times \mathrm{XO}^{1-\theta} \theta^{\theta} \rho^{1-\theta}\right]\right)$

\section{- Differentiate $K$, logarithmically}

dtest [4] = D[test [4], t] // Fullsimplify

$-\frac{\rho}{\theta}-\left(\mathrm{AKO}^{\theta} \mathrm{X} 0 \theta^{\theta} \rho\right) /\left(\mathrm{AKK} 0^{\theta} \mathrm{tX} 0(-1+\theta) \theta^{\theta} \rho-\mathrm{KOXO}^{\theta} \theta \rho^{\theta}\right)$ 


\section{Essay 2}

\section{Exhaustible Resources and}

\section{Technology Substitution in a}

\section{Model of Endogenous Growth}

\section{$2.1 \quad$ Introduction}

In 1972, Meadows et. al published the infamous book "The Limits to Growth," which predicts, with the help of a computer model, that the world economy will soon run out of key mineral resources and drown in pollution. Economists objected to the dire predictions of The Limits to Growth by claiming that new substitutes will be found for the minerals in short supplies, and technological progress will solve both the scarcity and the environmental problems. On October 7, 1973, the Arab member states of OPEC imposed an oil embargo on the US, Japan, and Western European countries. The effect upon oil-consuming nations was immediate, profound, and long-lasting: overnight, the price of a barrel of oil rose from $\$ 3$ to $\$ 5$. In response to The Limits to Growth and high energy prices, economists began to examine systematically the lim- 
its imposed by exhaustible resources and the environment on the process of economic growth, and whether growth is sustainable; see, for example, The Symposium on the Economics of Exhaustible Resources organized by The Review of Economic Studies (1974). The partial equilibrium framework in which Hotelling (1931) formulated his classic model of resource depletion now cedes its place to full dynamic general equilibrium macro-economic models. Dasgupta and Heal (1974) show that without technological progress sustainable production is not possible if the elasticity of substitution between the exhaustible resource and reproducible capital is less than unity. Stiglitz (1974) showed that sustained economic growth is possible if reproducible capital can be substituted for the essential exhaustible resource in question or if there is technological progress to counteract a dwindling resource stock. Solow (1974) also argued that the finite supply of an exhaustible resource does not necessarily constrain the growth of an economy if there is technological progress. With the advent of endogenous growth theory - in which technological progress occurs through learning by doing (Arrow (1962)) or through the accumulation of human capital (Lucas (1988)) - the recent research on exhaustible resources, the environment, and economic growth makes the link between the works of Stiglitz and Solow with the endogenous growth theory. The broadened view of reproducible capital - which now includes not only physical capital, but also human capital and knowledge - provides new perspectives on the substitution between exhaustible resources and reproducible capital, and makes sustained growth possible. Schou (1999), Scholz and Ziemes (1999), Aghion and Howitt (1998), Groth and Schou (2002) show that when the elasticity of substitution between the exhaustible resource and reproducible capital is equal to unity, sustained growth is possible. See also Smulders $(1999,2004)$ and Bretschger and Sjak Smulders (2003). The backstop technology, since its introduction by Nordhaus (1973) attracted authors to review the possibility of its integration in the DHSS model. Hoel 
(1978) and Stiglitz and Dasgupta (1982) reviewed the DHSS model in partial equilibrium settings and show that the relative price of the resource compared to the backstop technology increases over time and the backstop is adapted once prices are equalized. They investigated the impact of different market structures on the timing of backstop technology adoption and the development of extraction and the resource price over time. Barbier (1999) studied the role of endogenous technological change in alleviating resource scarcity. Scholz and Ziemes (1999) investigate the effect of monopolistic competition on steady state growth in a model with an essential nonrenewable resource. More recently Tsur and Zemel (2003) introduced R\&D, where the accumulation of knowledge gradually decreases the per unit cost of the backstop technology.

My contribution is the integration of the backstop technology in the DHSS model and completely characterize the optimal solution of the dynamic general equilibrium. In this paper, I formulate a dynamic general equilibrium macroeconomic model to study the depletion of an exhaustible resource, say fossil fuels, which has a substitute, renewable energy, produced by a backstop. The economy I consider has three sectors: a consumption good sector and two energy sectors - fossil fuels and a backstop. The consumption good is produced using labor, capital, and energy. The energy input used in the production of the consumption good can be obtained by burning fossil fuels or by operating the backstop. The backstop uses only capital to produce renewable energy from a linear technology. At each instant, the available capital stock is allocated between the consumption good sector and the backstop. The energy generated by fossil fuels and the energy produced by the backstop are assumed to be perfect substitutes. There is no population growth in the model. Technological progress occurs in the consumption good sector through learning by doing à la Arrow (1962), i.e., the capital stock at any instant embodies all the knowledge gained from 
past investments. Following Romer (1986), I assume that the output of the consumption good has a Cobb-Douglas structure with respect to the capital and energy inputs. The model I formalize thus has the structure of the AK model of endogenous growth, as formulated by Rebelo (1991). Although oil can be extracted at negligible cost, its stock is limited. The backstop, on the other hand, can produce a perpetual flow of energy. However, energy produced from the backstop requires capital - solar collectors, wind turbines. As oil resources dwindle, it is imperative that the backstop be brought into use to prevent a drastic reduction in consumption. The substitution of renewable energy for energy generated by burning fossil fuels is thus an important economic issue. In the paper, I analyze the pattern of resource depletion, the problem of technology substitution, and their impacts on economic growth. The model works in two phases. The first phase it operates as an DHSS model, but the resource stock is depleted in finite time instead of being meagerly stretched out until the economy collapses in the long run.Consumption rises as the resources reaches its lowest level, and then begins the ascent. By the time the stock of fossil fuels is depleted, consumption will have begun its climb, and immediately after depletion, the model switches to operate as an AK model. The paper is organized as follows. In Section 2, the model is presented. In the model, preferences are of the type with constant inter-temporal elasticity of substitution, and the dynamics of the system is homogeneous of degree one in control and state variables. The Hamilton-Jacobi-Bellman equation, which characterizes the optimal solution of the model, is given in Section 3. Section 4 contains the analysis of the economy on its balanced growth path after the stock of fossil fuels has been depleted. The analysis of the phase during which the stock of fossil fuels is exploited is given in Section 5. A characterization of the optimal solution is presented in Section 6. Section 7 contains a numerical example. Some concluding remarks are given in Section 8. 


\subsection{The model}

In the model, time is continuous and denoted by $t, t \geq 0$. The economy produces a consumption good, using three inputs - capital, labor, and a composite energy input. The composite energy input is generated by combining energy from two sources fossil fuels and a backstop. In what follows, I also refer to fossil fuels as oil.

\subsubsection{Preferences}

Let $u(c)$ denote the instantaneous utility of consumption, I assume that

$$
u(c)=\frac{c^{1-\theta}}{1-\theta}
$$

where $\theta \in(0,1)$, is a parameter, which is the inverse of the inter-temporal elasticity of substitution.

\subsubsection{The energy technologies}

The backstop produces renewable energy, using only capital. I assume that if $K_{0}$ is the capital input used in the backstop sector, then the amount of renewable energy measured in Btu - is equal to $\beta K_{0}$. That is, one unit of capital used in the backstop sector allows the economy to harness $\beta$ Btus from the Sun.

If $x$ is the amount of fossil fuel - expressed in Btus - burned at an instant, and $K_{0}$ is the capital input used in the backstop sector, then the composite energy input generated is assumed to be given by

$$
q=x+\beta K_{0}
$$

where $\beta>0$ is a constant. According to (2.2.2), energy obtained by burning fossil fuels and renewable energy are perfect substitutes for generating the composite energy input. 


\subsubsection{The consumption good technology}

The consumption good is produced according to the following technology:

$$
Y=A F\left(K_{1}, q, L\right)
$$

where $Y$ represents output, $K_{1}$ the capital input, $q$ the composite energy input, and $L$ the labor input. Also, $A$ is the technological level. The population is assumed to be constant and normalized to 1 . In what follows, I assume that $F\left(K_{1}, q, 1\right)$ has the Cobb-Douglas form:

$$
F\left(K_{1}, q, 1\right)=K_{1}^{\alpha} q^{1-\alpha}
$$

where $\alpha, 0<\alpha<1$, is a parameter. Under this assumption, the technology for producing the consumption good becomes

$$
Y=A F\left(K_{1}, q, 1\right)=A K_{1}^{\alpha} q^{1-\alpha}
$$

which implies that with a fixed labor input there is constant returns to scale of the other two factors - capital and energy. The production function represented by $(2.2 .5)$ is thus the technology of the AK model of endogenous growth a la Arrow (1962) and Romer (1986).

Let $K$ be the capital stock of the economy at any instant, the allocation of capital between the backstop sector and the consumption good sector at that instant satisfies the following identity:

$$
K=K_{0}+K_{1}
$$

This identity describes the collapse of the two capital stocks to one state variable, and find its explanation in the property of additivity of the two state variables, $K_{0}$ and $K_{1}$, combined with the linearity of the control variables. 


\subsubsection{Formal statement of the optimal growth problem with two energy sectors}

At each time $t$, the state of the system is represented by a list $(X(t), K(t))$, where $X(t)$ and $K(t)$ denote, respectively, the remaining stock of fossil fuels and the stock of capital. The central planner has to choose:

(i) an allocation of the capital stock $K(t)$ between the backstop sector $\left(K_{0}(t)\right)$ and the consumption good sector $K_{1}(t)$, with $K_{0}(t)+K_{1}(t)=K(t)$,

(ii) $c(t)$, the consumption,

(iii) $x(t)$, the oil extraction rate, and

(iv) $k(t)$, the capital investment

to maximize

$$
\int_{0}^{\infty} e^{-\rho t} u(c(t)) d t
$$

subject to

$$
\begin{gathered}
K_{0}(t)+K_{1}(t)=K(t), \\
A K_{1}^{\alpha}(t)\left(X(t)+\beta K_{0}(t)\right)^{1-\alpha}=c(t)+k(t), \\
\frac{d X}{d t} \frac{d X}{d t}=-x(t), \\
X(0)>0 \text { is given, } \\
\frac{d K}{d t}=k(t), \\
K(0)>0 \text { is given. }
\end{gathered}
$$

The collapse of the two capital stocks to one state variable find its explanation in the property of additivity of the two state variables, $K_{0}$ and $K_{1}$, combined with the 
linearity of the control variables. One may check, in our case, that the related costate variables to these two capitals are the same. If we introduced different adjustment costs for the capitals, we may have also have the collapsing property if the ratio of the costate variables $K_{0}$ and $K_{1}$ is a constant. For more discussion on this subject, see Pitchford and Turnovsky (1977). Taking into consideration the budget constraints (2.2.8) and (2.2.9), we can restate the central planner's problem under the following more compact form:

$$
\max _{\left(K_{1}(t), x(t), c(t)\right)} \int_{0}^{\infty} e^{-\rho t} u(c(t)) d t
$$

subject to

$$
\begin{gathered}
\frac{d X}{d t}=-x(t), \\
X(0)>0 \text { is given, } \\
\frac{d K}{d t}=A K_{1}^{\alpha}(t)\left(x(t)+\beta\left(K(t)-K_{1}(t)\right)\right)^{1-\alpha}-c(t), \\
K(0)>0 \text { is given. }
\end{gathered}
$$

A control policy $t \rightarrow\left(K_{1}(t), x(t), c(t)\right), t \geq 0$, is said to be admissible if it is nonnegative and piecewise right continuous. An admissible control is feasible if the solution of the system of differential equations that it induces is such that $X(t) \geq 0$, $K(t) \geq 0$. and $K_{1}(t) \leq K(t)$ for all $t \geq 0$.

\subsection{The Hamilton-Jacobi-Bellman equation}

Suppose that the economy begins at time $\tau \geq 0$ with $\xi$ as the remaining stock of oil and $\eta$ as the stock of capital. Let

$$
v(\xi, \eta, \tau)=\max _{\left(K_{1}(t), x(t), c(t)\right)_{t>\tau}} \int_{\tau}^{\infty} e^{-\rho t} u(c(t)) d t
$$


subject to

$$
\begin{gathered}
\frac{d X}{d t}=-x(t), \\
X(\tau)=\xi, \\
\frac{d K}{d t}=A K_{1}^{\alpha}(t)\left(x(t)+\beta\left(K(t)-K_{1}(t)\right)\right)^{1-\alpha}-c(t), \\
K(\tau)=\eta .
\end{gathered}
$$

As defined, $v(\xi, \eta, \tau)$ represents the optimal present value of social welfare, given that the economy begins at time $\tau$ with $\xi$ as the remaining stock of oil and $\eta$ as the stock of capital. Note that when $\xi=X(0), \eta=K(0)$, the preceding dynamic optimization problem is reduced to the central planner's original problem. The following lemma gives some useful properties of the optimal solution of the optimization problem constituted by (2.3.1) (2.3.3).

Lemma 1. The optimal-value function $v(\xi, \eta, \tau)$ has the following properties:

(i) It is homogeneous of degree $(1-\theta)$ in $(\xi, \eta)$ and for any $\tau$, the optimal control policy associated with $(\xi, \eta, \tau)$ is homogeneous of degree 1 in $(\xi, \eta)$

(ii) It is concave in $(\xi, \eta)$.

Proof. See Appendix A.

I would like to mention in passing that the homogeneity of the optimal-value function is well known in discrete-time dynamic programming; see, for example Stokey (1996), Alvarez and Stokey (1998), or Le Van and Morhaim (2002).

Suppose that the economy begins at time $t$, with $X$ as the remaining stock of oil and $K$ as the stock of capital. It is well known that the following Hamilton-JacobiBellman equation, which must be satisfied by the optimal-value function $v$ at each 
point $(X, K, t)$ :

$$
\begin{aligned}
0=\max _{\left(K_{1}, x, c\right)} & \left\{e^{-\rho t} \frac{c^{1-\theta}}{1-\theta}-D_{1} v(X, K . t) x\right. \\
+ & \left.D_{2} v(X, K . t)\left(A K_{1}^{\alpha}\left(x+\beta\left(K-K_{1}\right)\right)^{1-\alpha}-c\right)+D_{3} v(X, K, t)\right\} .
\end{aligned}
$$

Here $D_{1} v(X, K, l), D_{2} v(X, K, t)$, and $D_{3} v(X, K, t)$ denote, respectively, the partial derivatives of $v(X, K, l)$ with respect to $X, K$, and $t$. The values of $K_{1}, x$, and $c$ that solve the maximization problem in (2.3.4) constitute the optimal allocation of capital to the consumption good sector. The optimal oil extraction rate, and the optimal consumption - when the state of the system at time $t$ is $(X, K, t)$. Because the differcntial equations that govern the motion of the state variables are autonomous, and because time enters the objective function (2.3.1) only to discount utilities, I must have $v(X, K, t)=e^{-\rho t} v(X, K, 0)$. It follows immediately from this result that $D_{3} v(X, K, l)=-\rho v(X, K, l)$. In what follows, I will suppress time $(0)$ in the expression $v(X, K, 0)$, and, by abusing of notation, will simply write this expression as $v(X, K)$. The expression $v(X, K)$ thus represents the optimal present value of the stream of utilities, given that the system begins at time 0 with $X$ as the stock of oil and $K$ as the stock of capital. In addition, when $X=0$, when the stock of fossil fuels has been exhausted, I shall write $v(0, K)$ simply as $v(K)$. With this convention, the current-value version of the Hamilton-Jacobi-Bellman is thus given by

$$
\begin{array}{r}
0=\max _{\left(K_{1}, c, x\right)}\left\{\frac{c^{1-\theta}}{1-\theta}-D_{1} v(X, K) x+D_{2} v(X, K)\left(A K_{1}^{\alpha}\left(x+\beta\left(K-K_{1}\right)\right)^{1-\alpha}-c\right)\right. \\
-\rho v(X, K)\}
\end{array}
$$

Proposition 1. For any $X \geq 0, K>0$, the optimal value function has the following form:

$$
v(X, K)=K^{1-\theta} \omega\left(\frac{X}{K}\right)
$$


where $\omega: \lambda \rightarrow \omega(\lambda), \lambda \geq 0$, is an increasing and concave function of $\lambda$.

Proof. Because $v(X, K)$ is homogeneous of degree $(1-\theta)$ in $(X, K)$, we have

$$
\begin{aligned}
v(X, K) & =v\left(K\left(\frac{X}{K}, 1\right)\right) \\
& =K^{1-\theta} v\left(\frac{X}{K}, 1\right) \\
& =K^{1-\theta} \omega\left(\frac{X}{K}\right)
\end{aligned}
$$

where I define $\omega: \lambda \rightarrow \omega(\lambda)=v(\lambda, 1), \lambda \geq 0$.

According to Lemma $1, v(X, K)$ is concave in $(X, K)$. Hence, for a fixed $K$. $X \rightarrow v(X, K)$ is concave in $X$, and from this follows the concavity of $\omega(\lambda)$.

Corollary 1. For any given $X>0$, we have

$$
\lim _{K \rightarrow 0} \frac{D_{1} v(X, K)}{D_{2} v(X, K)}=0
$$

and

$$
\lim _{X \rightarrow 0} \frac{D_{1} v(X, K)}{D_{2} v(X, K)}=\infty .
$$

That is, the ratio of the shadow price of oil over the shadow price of capital tends to 0 when $K \rightarrow 0$, and tends to infinity when $X \rightarrow 0$.

Proof. Using the functional form (2.3.6) for $v(X, K)$, I obtain the following expressions for the shadow price of oil and the shadow price of capital, respectively, $D_{1} v(X, K)=K^{-\theta} \omega^{\prime}\left(\frac{X}{K}\right), D_{2} v(X, K)=K^{-\theta}(1-\theta) \omega\left(\frac{X}{K}\right)-K^{-1-\theta} X \omega^{\prime}\left(\frac{X}{K}\right)$. 
Thus,

$$
\begin{aligned}
\frac{D_{1} v(X, K)}{D_{2} v(X, K)} & =\frac{K^{-\theta} \omega^{\prime}\left(\frac{X}{K}\right)}{K^{-\theta}(1-\theta) \omega\left(\frac{X}{K}\right)-K^{-1-\theta} X \omega^{\prime}\left(\frac{X}{K}\right)} \\
& =\frac{1}{\frac{(1-\theta) \omega\left(\frac{X}{K}\right)}{\omega^{\prime}\left(\frac{X}{K}\right)}-\frac{X}{K}} \\
& =\frac{1}{\frac{(1-\theta) \omega(\lambda)}{\omega^{\prime}(\lambda]}-\lambda} \\
& =\frac{1}{\lambda\left(\frac{(1-\theta) \omega(\lambda)}{\lambda \omega^{\prime}(\lambda)}-1\right)},
\end{aligned}
$$

where I have let $\lambda=\frac{X}{K}$.

Now note that when $K \rightarrow 0$, I have $\lambda \rightarrow \infty$. Furthermore, because $\omega(\lambda) \rightarrow \infty$ and

$\omega^{\prime}(\lambda) \rightarrow 0$ when $\lambda \rightarrow \infty$, we must have $\frac{(1-\theta) \omega(\lambda)}{\lambda \omega^{\prime}(\lambda)}>1$, when $\lambda$ is large. Therefore, the expression on the last line of the preceding chain of equalities will tend to 0 when $K \rightarrow 0$. We have just established (2.3.7). To obtain (2.3.8), note that the two state variables $X$ and $K$ play symmetric roles in $v(X, K)$.

\subsection{The post-fossil fuel phase}

After the stock of fossil fuels has been depleted, we have $X=0$ and a fortiori $x=0$.

The Hamilton-Jacobi-Bellman equation (2.3.5) is then reduced to

$$
0=\max _{\left(K_{1}, c\right)}\left\{\frac{c^{1-\theta}}{1-\theta}+v^{\prime}(K)\left(A K_{1}^{\alpha}\left(0+\beta\left(K-K_{1}\right)\right)^{1-\alpha}-c\right)-\rho v(K)\right\} .
$$

The first-order condition characterizes the optimal consumption is

$$
0=c^{-\theta}-v^{\prime}(K)
$$

from which we obtain

$$
c=v^{\prime}(K)^{-\frac{1}{\theta}}
$$


The following first-order condition characterizes the optimal allocation of capital to the consumption good sector:

$$
0=v^{\prime}(K)\left(\alpha A K_{1}^{\alpha-1}\left(\beta\left(K-K_{1}\right)\right)^{1-\alpha}-\beta(1-\alpha) A K_{1}^{\alpha}\left(\beta\left(K-K_{1}\right)\right)^{-\alpha}\right)
$$

from which we obtain

$$
K_{1}=\alpha K
$$

The output of the consumption good, as a function of the capital stock, is given by

$$
\begin{aligned}
Y & =A K_{1}^{\alpha}\left(\beta K_{0}\right)^{1-\alpha} \\
& =A(\alpha K)^{\alpha}(\beta(1-\alpha) K)^{1-\alpha} \\
& =A K(1-\alpha)^{1-\alpha} \alpha^{\alpha} \beta^{1-\alpha}
\end{aligned}
$$

which indicates that the endogenous growth model with a renewable energy sector has the structure of the AK model of the endogenous growth literature; see, for example Rebelo (1991). Using (2.4.2), (2.4.3), and (2.4.4) in (2.3.5), we obtain

$$
0=\frac{1}{1-\theta} v^{\prime}\left(K^{\prime}\right)^{-\frac{1-\theta}{\theta}}+v^{\prime}(K)\left(A K(1-\alpha)^{1-\alpha} \alpha^{\alpha} \beta^{1-\alpha}-v^{\prime}(K)^{-\frac{1}{\theta}}\right)-\rho v(K) .
$$

According to Lemma $1, v(K)$ is homogeneous of degree $(1-\theta)$, and it follows from this property that $v(K)=\frac{1}{1-\theta} K v^{\prime}(K)$, which can then be used in (2.4.5) to obtain

$$
0=\frac{1}{1-\theta} v^{\prime}(K)^{-\frac{1-\theta}{\theta}}+v^{\prime}(K)\left(A K(1-\alpha)^{1-\alpha} \alpha^{\alpha} \beta^{1-\alpha}-v^{\prime}(K)^{-\frac{1}{\theta}}\right)-\rho \frac{1}{1-\theta} K v^{\prime}(K),
$$

which can be simplified to

$$
0=\frac{\theta}{1-\theta} v^{\prime}(K)^{-\frac{1}{\theta}}+A K(1-\alpha)^{1-\alpha} \alpha^{\alpha} \beta^{1-\alpha}-\frac{\rho}{1-\theta} K
$$

or

$$
v^{\prime}(K)=K^{-\theta}\left(\frac{1}{\theta}\left(\rho-A(1-\alpha)^{1-\alpha} \alpha^{\alpha} \beta^{1-\alpha}(1-\theta)\right)\right)^{-\theta}
$$


Integrating the preceding differential equation and using the boundary condition $v(0)=0$, we obtain

$$
v(K)=\frac{1}{1-\theta} K^{1-\theta}\left(\frac{1}{\theta}\left(\rho-A(1-\alpha)^{1-\alpha} \alpha^{\alpha} \beta^{1-\alpha}(1-\theta)\right)\right)^{-\theta} .
$$

Because $v(K)>0$ when $K>0$, the parameters must satisfy the following condition: (30)

$$
\rho-(1-\theta) A \alpha^{\alpha}(\beta(1-\alpha))^{1-\alpha}>0 .
$$

Using (2.4.7) to evaluate (2.4.2), we obtain the following expression for the optimal consumption, as a function of the capital stock:

$$
c=K\left(\frac{1}{\theta}\left(\rho-A(1-\alpha)^{1-\alpha} \alpha^{\alpha} \beta^{1-\alpha}(1-\theta)\right)\right)
$$

Using (2.4.4) and (2.4.9), we obtain the following differential equation, which governs the motion of the stock of capital through time:

$$
\begin{aligned}
\frac{K^{\prime}(t)}{K(t)} & =\left(A \alpha^{\alpha}(\beta(1-\alpha))^{1-\alpha}-\frac{1}{\theta}\left(\rho-A(1-\alpha)^{1-\alpha} \alpha^{\alpha} \beta^{1-\alpha}(1-\theta)\right)\right) \\
& =\frac{1}{\theta}\left(A(1-\alpha)^{1-\alpha} \alpha^{\alpha} \beta^{1-\alpha}-\rho\right)
\end{aligned}
$$

which asserts that the stock of capital grows through time at the constant rate

$$
\gamma=\frac{A(1-\alpha)^{1-\alpha} \alpha^{\alpha} \beta^{1-\alpha}-\rho}{\theta} .
$$

Because at each instant a proportion $\alpha$ of the capital stock is allocated to the consumption good sector and a proportion $(1-\alpha)$ of the capital stock is allocated to the renewable energy sector, the capital input in each sector also grows through time at rate $\gamma$. Finally, according to (2.4.9), consumption at any instant is a constant proportion of the capital stock. Hence consumption also grows at rate $\gamma$. It follows from (2.4.8) and (2.4.11) that the following inequalities must be satisfied so that the 
economy grows along a balanced growth path after the stock of fossil fuels has been depleted

$$
(1-\theta) A \alpha^{\alpha} \beta^{1-\alpha}(1-\alpha)^{1-\alpha} \alpha^{\alpha}<\rho<A \beta^{1-\alpha}(1-\alpha)^{1-\alpha} \alpha^{\alpha}
$$

I summarize the results just obtained in the following proposition:

Proposition 2. Immediately after the depletion of the stock of fossil fuels, the economy enters a steady state of balanced growth. In the steady state, the capital stock, the output of renewable energy, and consumption all grow at the same rate.

\subsection{The fossil fuel phase}

Let $\left(K_{1}^{*}(t), x^{*}(t), c^{*}(t)\right), t \geq 0$, be the solution of the central planner's problem, as stated by (2.2.12) (2.2.14). Under the optimal controls, the motion of the system is governed by the following differential equations:

$$
\begin{gathered}
\frac{d X^{*}}{d t}=-x^{*}(t), \\
X^{*}(0)>0 \text { is given, } \\
\frac{d K^{*}}{d t}=A\left(K_{1}^{*}(t)\right)^{\alpha}\left(x^{*}(t)+\beta\left(K^{*}(t)-K_{1}^{*}(t)\right)\right)^{1-\alpha}-c^{*}(t), \\
K^{*}(0)>0 \text { is given. }
\end{gathered}
$$

Next, let

$$
\begin{aligned}
& \phi(t)=D_{1} v\left(X^{*}(t), K^{*}(t), t\right), \\
& \varphi(t)=D_{2} v\left(X^{*}(t), K^{*}(t), t\right) .
\end{aligned}
$$

denote, respectively, the shadow price of oil and the shadow price of capital - both at time $t$ along the optimal trajectory. Applying (2.3.4) at an instant $t$ along the 
optimal trajectory, we can assert that

$$
\begin{aligned}
\left(K_{1}^{*}(t), x^{*}(t), c^{*}(t)\right) & =\operatorname{argmax}_{\left(K_{1}, c, x\right)}\left\{e^{-\rho t} \frac{c^{1-\theta}}{1-\theta}-\phi(t) x\right. \\
& \left.+f(t)\left(A K_{1}^{-\alpha}\left(x+\beta\left(K^{*}(t)-K_{1}\right)\right)^{1-\alpha}-c\right)\right\} \\
& =\operatorname{argmax}_{\left(K_{1}, c, x\right)} \mathcal{H}\left(X^{*}(t), K^{*}(t), K_{1}, x, c, \phi(t), \varphi(t), t\right),
\end{aligned}
$$

where the Hamiltonian $\mathcal{H}$ is defined by

$\mathscr{H}\left(X, K, K_{1}, x, c, \phi, \varphi, t\right)=e^{-\rho t} \frac{c^{1-\theta}}{1-\theta}-\phi(t) x+\varphi(t)\left(A K_{1}^{\alpha}\left(x+\beta\left(K-K_{1}\right)\right)^{1-\alpha}-c\right)$

The motion of the shadow prices of oil, physical capital and backstop are derived in the appendix $\mathrm{B}$. The motion of $\phi(t)$ is governed by the following differential equation:

$$
\frac{d \phi}{d t}=-D_{1} \mathscr{H}\left(X^{*}(t), K^{*}(t), K_{1}^{*}(t), x^{*}(t), c^{*}(t), \phi(t), \varphi(t), t\right)=0
$$

which implies that the shadow price of oil is constant along the optimal trajectory, say $\phi(t)=\phi=$ constant, $t \geq 0$. The motion of $\varphi(t)$ is governed by the following differential equation:

$$
\begin{aligned}
\frac{d \varphi}{d t} & =-D_{2} \mathcal{H}\left(X^{*}(t), K^{*}(t), K_{1}^{*}(t), x^{*}(t), c^{*}(t), \phi(t), \varphi(t), t\right) \\
& =-\varphi(t)\left(A\left(K_{1}^{*}(t)\right)^{\alpha}\left(x^{*}(t)+\beta\left(K^{*}(t)-K_{1}^{*}(t)\right)\right)^{-\alpha}(1-\alpha) \beta\right) .
\end{aligned}
$$

Note that the shadow price of capital is falling along the optimal trajectory. The value of $c$ that maximizes the Hamiltonian is

$$
c^{*}(t)=\left(e^{\rho t} \varphi(t)\right)^{-\frac{1}{\theta}}
$$

The following first-order conditions characterizes the optimal allocation of capital to the sector producing the consumption good:

$$
A K_{1}^{-(1-\alpha)} \varphi(t)\left(x \alpha-\beta K_{1}+\alpha \beta K^{*}(t)\right)\left(x+\beta\left(K^{*}(t)-K_{1}\right)\right)^{-\alpha} \geq 0
$$


with equality holding if $K_{1}^{*}(t)<K^{*}(t)$. The following first-order conditions characterizes the optimal extraction rate:

$$
-\phi+A(1-\alpha) K_{1}^{\alpha} \varphi(t)\left(x+\beta\left(K^{*}(t)-K_{1}\right)\right)^{-\alpha} \leq 0
$$

with equality holding if $x^{*}(t)>0$.

Lemma 2. At any time, only one source of energy - fossil fuels or renewable energy, but not both - is exploited to provide the energy used in the production of the consumption good.

This lemma can be explained easily if we recall that energy composite input is a linear function of the oil and backstop. This perfect substitution feature dictates that only the cheapest one will be used. Until our days, it is well known that clean energy is costly and very difficult to obtain and manipulate than oil, the flexibility and usage is well established at all economic levels. Since oil was discovered first, it will be the first source of energy-fossil fuel era.

Proof. Lemma 2 is proved by reduction ad absurdum. Suppose that for some time $t$, we have both $x^{*}(l)>0$ and $K_{1}^{*}(t)<K^{*}(t)$. That is, the solution of the maximization of the Hamiltonian is an interior solution. Under such a scenario, (2.5.10) and (2.5.11) both hold with equality:

$$
\begin{gathered}
A K_{1}^{-(1-\alpha)} \varphi(t)\left(x \alpha-\beta K_{1}+\alpha \beta K^{*}(t)\right)\left(x+\beta\left(K^{*}(t)-K_{1}\right)\right)^{-\alpha}=0 \\
-\phi+A(1-\alpha) K_{1}^{\alpha} \varphi(t)\left(x+\beta\left(K^{*}(t)-K_{1}\right)\right)^{-\alpha}=0
\end{gathered}
$$

Solving (2.5.12) for $K_{1}$, we obtain

$$
K_{1}=\frac{x \alpha+\alpha \beta K^{*}(t)}{\beta}
$$

Using (2.5.14) in (2.5.12), we obtain after some simplifications

$$
-\phi+A(1-\alpha)^{1-\alpha} \frac{\alpha}{\beta}^{\alpha} \varphi(t)=0 .
$$


Because the controls are piecewise and right-continuous, (2.5.15) must hold in a right neighborhood of $t$. However, I have already indicated that the shadow price of capital is strictly decreasing through time. Thus, (2.5.15) cannot hold in a right neighborhood of $t$.

Lemma 3. Consider a non-degenerate interval $[a, b), a \geq 0$, of the real line. If the stock of fossil fuels is exploited at each instant during this time interval, i.e., if $x^{*}(t)>$ 0 for all $t \in(a, b)$, then the shadow price of capital during this time interval has the following form:

$$
\varphi(t)=-\beta \cdot \phi \cdot t+\zeta, \quad a \leq t<b
$$

where $\zeta$ is a constant of integration.

Proof. According to Lemma 2, only one energy technology is exploited at any instant. Thus, if $x^{*}(t)>0, a \leq t<b$, then the backstop will not be exploited at any point in time during this time interval. Thus, for each $t$ during this time interval, we have $K_{1}^{*}(t)=K^{*}(t)$, and the first-order condition (2.5.11), with $K_{1}^{*}(t)=K^{*}(t)$, will hold with equality:

$$
0-\phi+A(1-\alpha)\left(K^{*}(t)\right)^{\alpha} \varphi(t)\left(x^{*}(t)\right)^{-\alpha}=0
$$

from which we obtain

$$
x^{*}(t)=\left(\frac{A(1-\alpha) \varphi(t)}{\phi}\right)^{\frac{1}{\alpha}} K^{*}(t)
$$

Setting $K_{1}^{*}(t)=K^{*}(t)$ in (2.5.8), and then using (2.5.13) in the result, we obtain the following differential equation, which governs the motion of the shadow price of capital before the stock of fossil fuels is depleted:

$$
\begin{aligned}
\frac{d \varphi}{d t} & =-\varphi(t)\left(A\left(K^{*}(t)\right)^{\alpha}\left(x^{*}(t)\right)^{-\alpha}(1-\alpha) \beta\right) \\
& =-\varphi(t)(A(\phi /(A(1-\alpha) \varphi(t)))(1-\alpha) \beta) \\
& =-\beta \cdot \phi .
\end{aligned}
$$


Integrating this differential equation, we obtain the following expression for the shadow price of capital during the time interval $a \leq t<b$ :

$$
\varphi(t)=-b \cdot \phi \cdot l+\zeta
$$

where $\zeta$ is the constant of integration.

Proposition 3. The backstop will not be exploited until the stock of fossil fuels has been depleted.

Proof. Proposition 3 is proved by reduction ad absurdum. Suppose that the backstop is exploited at some time $t$ at which the stock of fossil fuels has not been exhausted, i.e., $K_{1}^{*}(t)<K^{*}(t)$ and $X^{*}(t)>0$. According to Lemma 2, the fossil fuel technology is not exploited at time $t: x^{*}(t)=0$. Using $x=x^{*}(t)=0$ in (2.5.10), which holds with equality at time $t$ because $K_{1}^{*}(t)<K^{*}(t)$, we obtain

$$
K_{1}^{*}(t)=\alpha K^{*}(t)
$$

Because the controls are piecewise and right continuous, I must have

$$
\begin{aligned}
K_{1}^{*}(s) & =\alpha K^{*}(s), \\
x^{*}(s) & =0, \quad t \leq s<t+\epsilon,
\end{aligned}
$$

where $\epsilon$ is a small positive number. Because $X^{*}(t)>0$, and because under the optimal solution the stock of fossil fuels must be depleted, the extraction rate must be positive at some time after $t+\epsilon$. Thus, if I let

$$
\tau=\inf \left\{s \geq t \mid x^{*}(s)>0\right\}
$$

denote the first time after $t$ the extraction rate is positive, then I must have

$$
K_{1}^{*}(\tau)=K^{*}(\tau), \quad x^{*}(\tau)=\left(\frac{A(1-\alpha) \varphi(\tau)}{\phi}\right)^{\frac{1}{\alpha}} K^{*}(\tau)>0
$$


That is, the fossil fuel technology is exploited in a right neighborhood of $\tau$. It also follows from the definition of $\tau$ that the backstop is exploited during the time interval $[t, \tau)$. By continuity, at the interface between the two regimes, i.e., at time $\tau$, either one of the two technologies can be exploited under the optimal solution. More precisely, at time $\tau$, the policy represented by $(2.5 .24)$ as well as the policy that is the limit of (2.5.22) when $s \uparrow \tau$, namely

$$
\lim _{s \uparrow \tau} K_{1}^{*}(s)=\alpha K^{*}(\tau), \quad \lim _{s \uparrow \tau} x^{*}(s)=0
$$

both maximize the Hamiltonian at time $\tau$, and this means

$$
\begin{aligned}
& \mathcal{H}\left(X^{*}(\tau), K^{*}(\tau), \alpha K^{*}(\tau), 0, c^{*}(\tau), \phi, \varphi(\tau), \tau\right) \\
= & e^{-\rho t} \frac{1}{1-\theta} c^{*}(\tau)^{1-\theta}+\varphi(\tau)\left(A\left(\alpha K^{*}(\tau)\right)^{\alpha}\left(\beta(1-\alpha) K^{*}(\tau)\right)^{1-\alpha}-c^{*}(\tau)\right) \\
0= & \mathcal{H}\left(X^{*}(\tau), K^{*}(\tau), K^{*}(\tau), x^{*}(\tau), c^{*}(\tau), \phi, \varphi(\tau), \tau\right) \\
= & e^{-\rho t} \frac{1}{1-\theta} c^{*}(\tau)^{1-\theta}-\phi\left(\frac{A(1-\alpha) \varphi(\tau)}{\phi}\right)^{\frac{1}{\alpha}} K^{*}(\tau) \\
& +\varphi(\tau)\left(A\left(K^{*}(\tau)\right)^{\alpha}\left(\left(\frac{A(1-\alpha) \varphi(\tau)}{\phi}\right)^{\frac{1}{\alpha}} K^{*}(\tau)\right)^{1-\alpha}-c^{*}(\tau)\right)
\end{aligned}
$$

which, after some simplifications, is reduced to

$$
\frac{A(1-\alpha)^{1-\alpha} \alpha^{\alpha} \beta^{-\alpha} \varphi(\tau)}{\phi}=1
$$

After time $\tau$, there are two possibilities to imagine: (i) $x^{*}(s)>0$ for all $s \geq \tau$, and (ii) $x^{*}(s)=0$ for some $s>\tau$. Under possibility (i), invoke Lemma 3 to assert that the shadow price of capital will become negative when $s$ is large, and this is absurd. Under possibility (ii), let $\hat{\tau}$ be the first time after $\tau$ that the backstop is exploited again. At the interface $\hat{\tau}$, the same argument used to obtain (2.5.2 7 ) can be deployed to obtain the following version of $(2.5 .27)$

$$
\frac{A(1-\alpha)^{1-\alpha} \alpha^{\alpha} \beta^{-\alpha} \varphi(\hat{\tau})}{\phi}=1
$$


However, we know that the shadow price of capital falls through time, and thus not both (2.5.27) and (2.5.28) can both hold. The premise of the reductio ad absurdum argument leads to a contradiction.

According to Proposition 3, the technology substitution is abrupt. The stock of fossil fuels is exploited first, and then upon its exhaustion, the backstop is brought into use. Once the backstop is brought into use, the economy instantaneously enters the steady state and evolves along a sustainable balanced growth path along which the capital stock, the output of renewable energy, and consumption all grow at the same constant rate. A transition phase during which the backstop gradually displaces fossil fuels in meeting the energy needs of the economy does not exist. The intuition behind this result is not hard to grasp. Because of the assumption that capital is freely mobile between the renewable and consumption good sectors, renewable energy can be produced at any instant by allocating capital to the backstop. Furthermore, while the maximum possible output of renewable energy that can be produced by the backstop is constrained by the stock of capital available at that instant, any amount of energy can be generated by burning fossil fuels at that instant. Thus, fossil fuels are more flexible than renewable energy, and this advantage favors the exploitation of the fossil fuel technology first.

Proposition 4. The stock of fossil fuels is depleted in finite time.

Proof. According to Proposition 3, the backstop will not be exploited before the stock of fossil fuels is depleted. Furthermore, because the shadow price of capital is positive, and its motion is governed by (2.5.16) of Lemma 3 as long as the fossil fuel technology is exploited. Thus, we must have $-b \phi t+\zeta>0$, i.e., $t<\frac{\zeta}{b \phi}<\infty$, and this means that the stock of fossil fuels must be depleted in finite time under the optimal solution.

Let $T$ denote the time at which the stock of fossil fuels is exhausted. According 
to Lemma 3, the shadow price of capital before oil exhaustion is given by

$$
\varphi(t)=-\beta \phi t+\varphi(0), \quad 0 \leq t<+T
$$

Note that in $(2.5 .29), \varphi(0)$ is the initial shadow price of capital, which is the particular value for the constant of integration $\zeta$ in (2.5.16). Using (2.5.29) in (2.5.9) and (2.5.12), we obtain, respectively, the following expressions for the optimal consumption and the optimal extraction rate at an instant before oil exhaustion:

$$
c^{*}(t)=e^{-\frac{\rho}{\theta} t}(-\beta \phi t+\varphi(0))^{-\frac{1}{\theta}}, \quad 0 \leq t \leq T
$$

and

$$
x^{*}(t)=\left(\frac{A(1-\alpha)}{\phi}\right)^{\frac{1}{\alpha}}(-\beta \phi t+\varphi(0))^{\frac{1}{\theta}} K^{*}(t), \quad 0 \leq t \leq T .
$$

The output of the consumption good at an instant before resource exhaustion is given by

$$
\begin{aligned}
Y^{*}(t) & =A\left(K^{*}(t)^{\alpha}\right)\left(x^{*}(t)\right)^{1-\alpha} \\
& =A\left(K^{*}(t)^{\alpha}\right)\left(\left(\frac{A(1-\alpha)}{\phi}\right)^{\frac{1}{\alpha}}(-\beta \phi t+\varphi(0))^{\frac{1}{\theta}} K^{*}(t)\right)^{1-\alpha} \\
& =A^{\frac{1}{\alpha}} K^{*}(t)\left(\frac{1-\alpha}{\phi}\right)^{\frac{1-\alpha}{\alpha}}(-\beta \phi t+\varphi(0))^{\frac{1-\alpha}{\theta}}, \quad 0 \leq t \leq T .
\end{aligned}
$$

The following differential equation governs the motion of the stock of capital before oil exhaustion:

$$
\begin{aligned}
\frac{d K^{*}}{d t} & =A^{\frac{1}{\alpha}} K^{*}(t)\left(\frac{1-\alpha}{\phi}\right)^{\frac{1 \alpha \alpha}{\alpha}}(-\beta \phi t+\varphi(0))^{\frac{1 \alpha \alpha}{\theta}}-e^{-\frac{\rho}{\theta} l}(-\beta \phi t+\varphi(0))^{-\frac{1}{\theta}}, \\
& \leq \leq t \leq T, \quad K^{*}(0)>0 \text { is given. }
\end{aligned}
$$

Note that (2.5.33) is a first-order linear differential equation in $K(t)$, and can be solved by using the integrating factor technique. In Appendix B., we obtain the following 
expression for the capital stock $K^{*}(t)$ before the stock of fossil fuels is depleted:

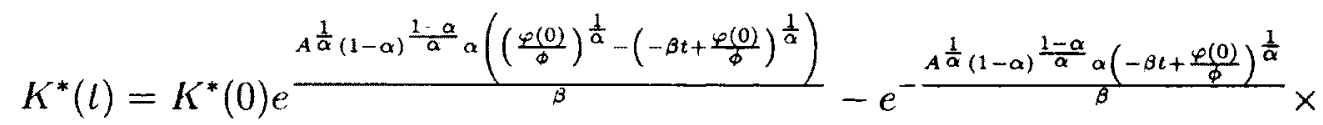

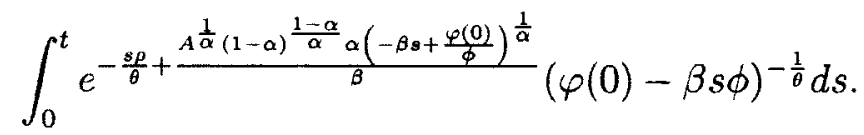

The stock constraint is given by

$$
\begin{aligned}
X_{0} & =\int_{0}^{T} x^{*}(t) d t \\
& =\left(\frac{A(1-\alpha)}{\phi}\right)^{\frac{1}{\alpha}} \int_{0}^{T}(-\beta \phi t+\varphi(0))^{\frac{1}{\theta}} K^{*}(t) d t
\end{aligned}
$$

Note that (2.5.34) determines the time path of the capital stock, given the values of $\phi$ and $\varphi(0)$. Using (2.5.34), we can compute $K^{*}(T)$, the capital stock at the date of exhaustion. The stock constraint (2.5.35) links $\phi$ and $\varphi(0)$. Thus, to find these two values, we need another equation. In addition, we must also determine $T$, the date of oil exhaustion. Thus, in total, we need two more equations. The following version of (2.5.27) - at the date of exhaustion - gives one needed equation

$$
\frac{A(1-\alpha)^{1-\alpha} \alpha^{\alpha} \beta^{-\alpha} \varphi(t)}{\phi}=\frac{1}{\phi} A(1-\alpha)^{1-\alpha} \alpha^{\alpha} \beta^{-\alpha}(-\beta \phi T+\varphi(0))=1 .
$$

The second needed equation involves the date of exhaustion and the stock of capital at that date:

$$
\varphi(t)=(-\beta \phi T+\varphi(0))=(1-\theta) K^{*}(T)^{-\theta} \omega(0)
$$

Together, using the expression for $K^{*}(T)$, which involves $T, \phi$, and $\varphi(0)$ - in (2.5.37), we can express this equation in the three unknowns $T, \phi, \varphi(0)$. This last equation, together with (2.5.36) and (2.5.37) constitutes a system of three equations in the three unknowns $T, \phi$, and $\varphi(0)$, whose solution determines completely the behavior of the economy during the phase the fossil fuel technology is exploited.

In the appendix $2 C$ we derive the closed form solution for the capital. Notice that 
this model, differs from the original DHSS model by the integration of the backstop technology, allowing for the mobility of capital between the sector of energy and the consumption production sector. The main feature of this model, is that it works in tow switching regimes. The first one is similar to the DHSS model, but before oil exhaustion, consumption will reach a global minimum and starts ascending. At the moment of exhaustion, an abrupt regime switching occurs, allowing the model to operate as an AK model. There was no need to assume the equality between $\theta$ and $\alpha$ to see this regime switching (see the closed form solution in the appendix). The following analysis clarifies this pattern by studying the consumption pattern.

To study the pattern of consumption before oil exhaustion, differentiate (2.5.30) logarithmically to obtain its growth rate:

$$
\begin{aligned}
\frac{1}{c^{*}(t)} \frac{d c^{*}}{d t} & =\frac{1}{\theta}\left(-\rho+\frac{\beta \phi}{(-\beta \phi t+\varphi(0))}\right) \\
& =\frac{1}{\theta} \psi(t), \quad 0 \leq t \leq T,
\end{aligned}
$$

where we have let

$$
\psi(t)=-\rho+\frac{\beta \phi}{(-\beta \phi t+\varphi(0))}, \quad 0 \leq t \leq T
$$

Proposition 5. The optimal pattern of consumption before the stock of fissile fuels is exhausted has the following properties:

(i) It is rising when the resource is near exhaustion.

(ii) If capital is scarce, but resources are abundant, then the optimal consumption is falling at the beginning, descends to its lowest level, and then rises until the time the stock of fossil fuels is exhausted.

(iii) If resources are scarce, but capital is abundant, then consumption rises continuously during the fossil fuel phase. 
Proof.

(i) At the date of resource exhaustion, we have

$$
\psi(t)=-\rho+\frac{\beta \phi}{\varphi(t)}
$$

Furthermore, using (2.5.36) in (2.5.40), we obtain

$$
\psi(t)=-\rho+\frac{\beta \phi}{\varphi(t)}=A(1-\alpha)^{1-\alpha} \alpha^{\alpha} \beta^{1-\alpha}-\rho>0
$$

where the strict inequality is due to (2.4.12), the condition imposed on the parameters so that balanced growth takes place after resource exhaustion.

(ii) If capital is scarce, but resources are abundant, then at time $0(2.5 .39)$ is reduced to

$$
\psi(0)=-\rho+\frac{\beta \phi}{\varphi(0)}<0
$$

The strict inequality is obtained by using the hypothesis that capital is scarce and (2.3.7) of the corollary. By continuity, $\psi(t)<0$ for $t$ in a right neighborhood of 0 , and this means the optimal consumption is falling initially. The optimal consumption descends to its lowest level, when $\psi(t)$ rises to 0 at time

$$
t=-\frac{1}{\rho}+\frac{\varphi(0)}{\beta \phi}
$$

(iii) If capital is abundant, but resources are scarce, then at time $0,(2.5 .39)$ is reduced to

$$
\psi(0)=-\rho+\frac{\beta \phi}{\varphi(0)}>0 .
$$

The strict inequality is obtained by using the hypothesis that resources are scarce and (2.3.8) of the corollary. In this case, $\psi(t)>0,0<t \leq T$, and, consequently, consumption rises continuously during the fossil fuel phase. 


\subsection{A characterization of the optimal solution}

The solution of the optimal endogenous growth model with an exhaustible resource and technology substitution can be characterized as follows. The fossil fuel technology will be exploited first, and the stock of fossil fuels is depleted in finite time. During this phase capital is built up, with oil being transformed into capital and consumption. The consumption pattern depends on the scarcity and abundance of capital and resources. If capital is scarce, but resources are abundant, consumption will be restrained initially to speed up the accumulation of capital. More precisely, consumption falls initially as time goes on, reaches its lowest level, and then begins the ascent. By the time the stock of fossil fuels is depleted, consumption will have begun its climb. On the other hand, if resources are scarce, but capital is abundant, consumption will rise continuously throughout the fossil fuel phase. Immediately after the stock of fossil fuels has been exhausted, the economy enters a steady state and grows along a balanced growth path.

\subsection{A numerical example}

For this numerical example, the parameters assume the following values $A=\frac{5}{2}, b=$ $\frac{3}{4}, \alpha=\frac{1}{2}, \theta=\frac{1}{2}, \rho=1$. The value of the resource stock is $X_{0}=0.647697$ and that of the capital stock is $K_{0}=0.227$. In steady state, the economy grows along a balanced growth path at rate

$$
\gamma=\frac{A(1-\alpha)^{1-\alpha} \alpha^{\alpha} \beta^{1-\alpha}-\rho}{\theta}=0.4
$$


The post-fossil fuel Bellman function is

$$
\begin{aligned}
v(K) & =\omega(0) K^{1-\theta} \\
& =-\frac{\theta^{\theta}\left(A(1-\alpha)^{1-\alpha} \alpha 7^{\alpha} \beta^{1-\alpha}(\theta-1)+\rho\right)^{-\theta}}{\theta-1} K^{1-\theta}=2.08802 K^{1-\theta} .
\end{aligned}
$$

The date at which the stock of fossil fuels is exhausted is $T=1$. Figure 2.1 below depicts the time path of the extraction rate.

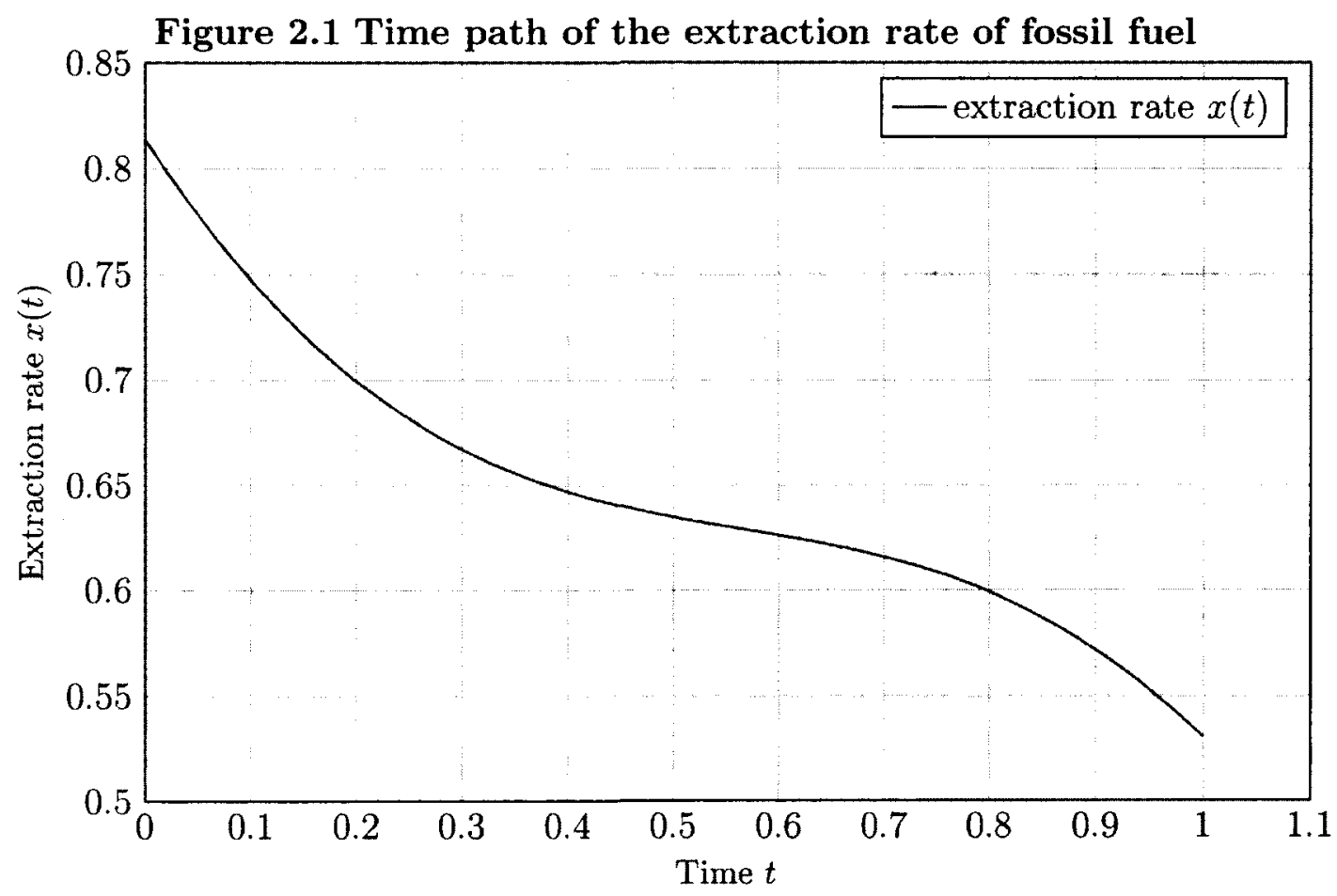

As can be see from the figure, the rate of extraction is steadily falling through time. Figure 2.2 depicts the time path of the capital stock during the fossil fuel phase. As can be seen from the figure, the capital stock rises during the fossil fuel phase. Figure 2.3 depicts the time path of consumption during the fossil fuel phase.

The figure indicates that the optimal consumption declines at the beginning, reaches its lowest level, and then rises during the last stage of the fossil fuel phase. 
Figure 2.2 Time path of physical capital accumulation

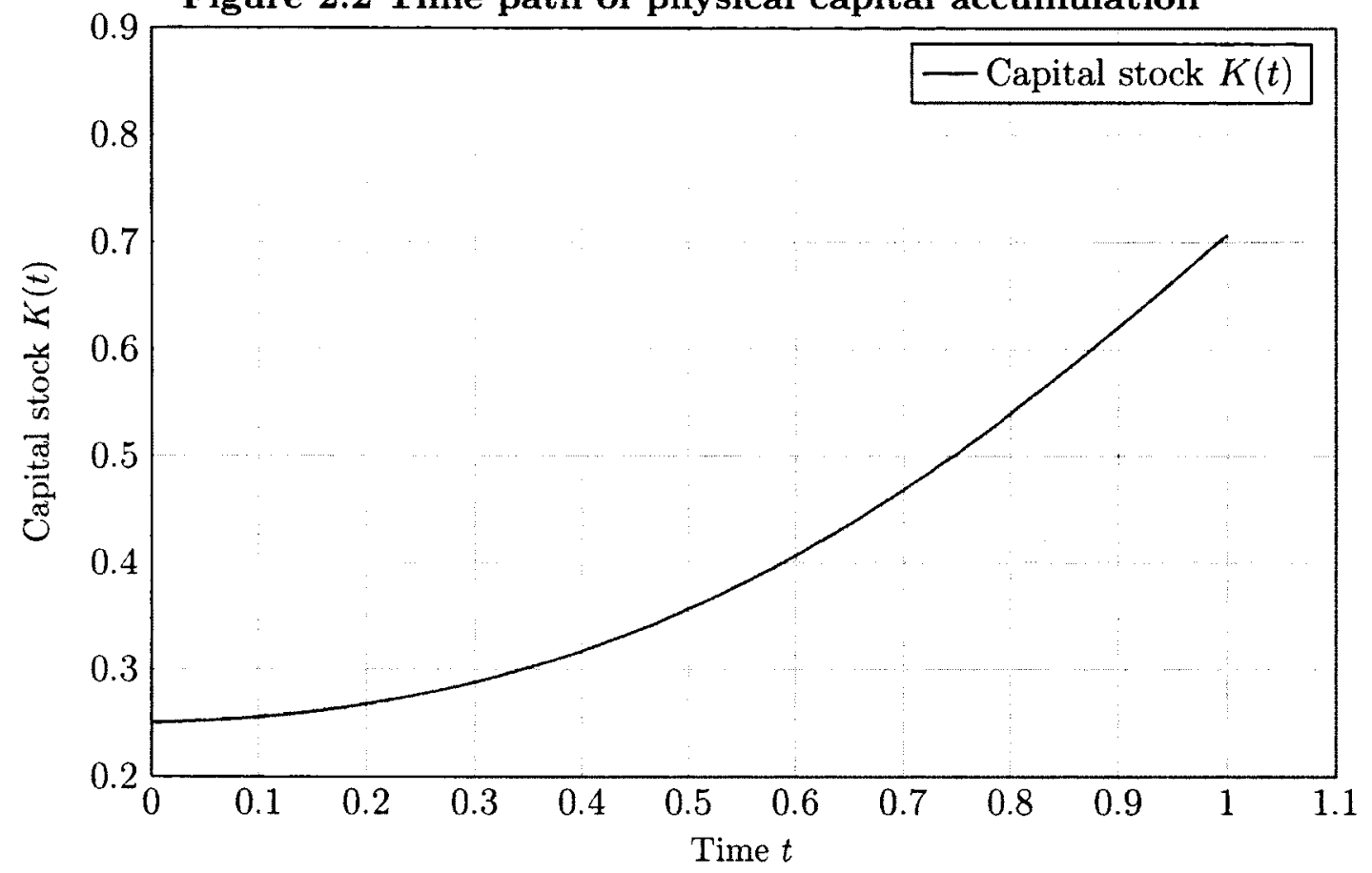

Figure 2.3 Time path of consumption

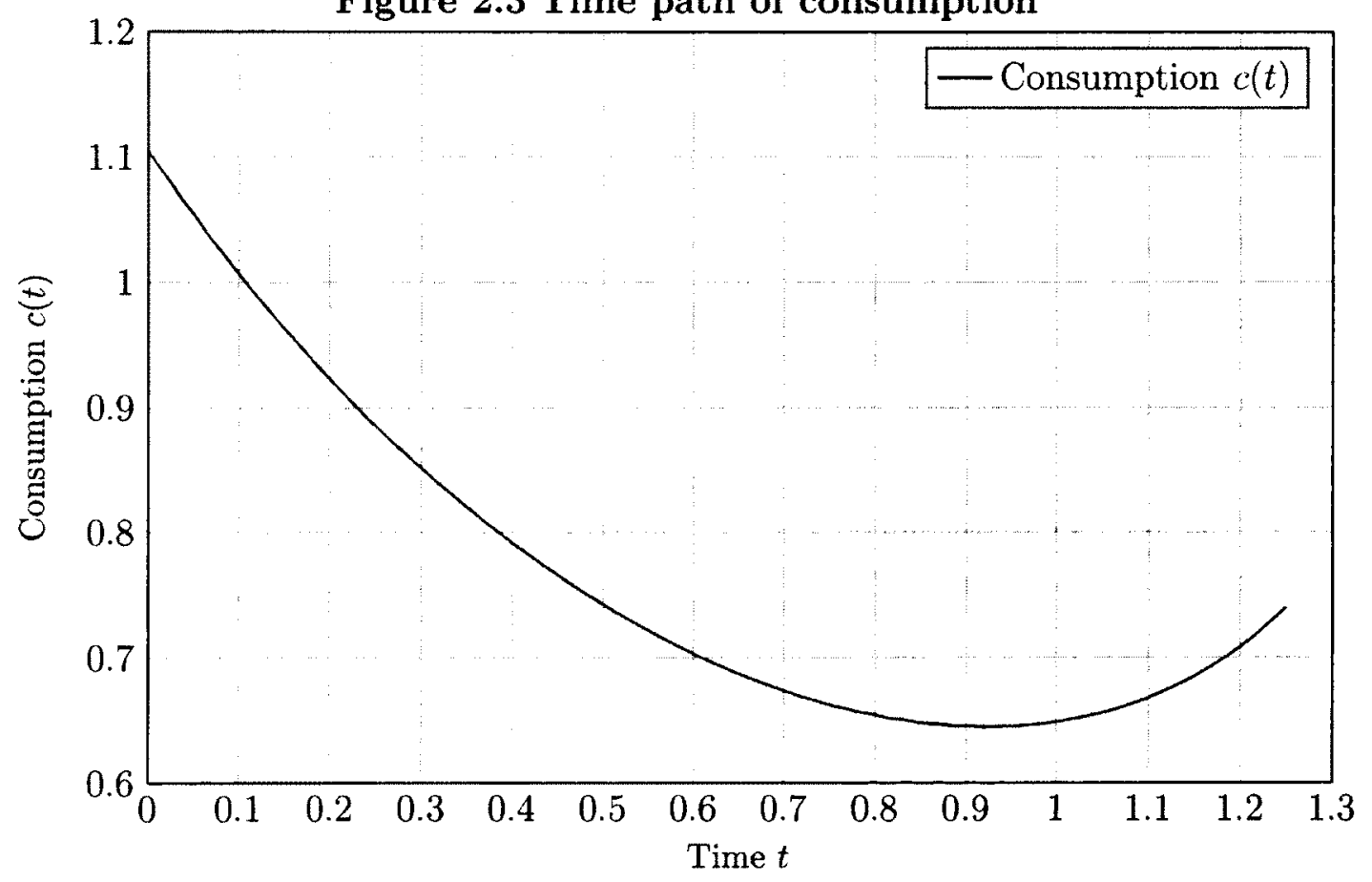




\subsection{Concluding remarks}

In this paper, I have formalized a model of endogenous growth with an exhaustible resource in which the resource has a perfect substitute produced by a backstop. The model thus represents an attempt made by several other researchers to link the literature on growth with exhaustible resources and the endogenous growth theory. What differentiates our model from the other models that attempt to make the link is that I provide a possible technology substitution for the exhaustible resource. In the classic model of resource depletion of Dasgupta and Heal (1974), the economy collapses in the long run when the resource runs out. The literature on endogenous growth and exhaustible resources attempt to demonstrate that endogenous technological progress can counteract the negative impact effect of resource depletion and allow for a sustainable or rising level of consumption. Such a scenario is possible when the possibility of technology substitution exists, as is the case of our model.

In the present model, the resource stock is exploited first, the backstop is only brought into use after the resource stock has been exhausted. In contrast with the model of Dasgupta and Heal (1974), the resource stock is depleted in finite time instead of being meagerly stretched out until the economy collapses in the long run. Another contrast with the model of Dasgupta and Heal (1974) is that in their model consumption is expected to fall through time to zero. Depending on the parameters of the model, consumption might rise at first, reach a peak, and then decline, as shown by Pezzy and Withagen (1998) and Hartwick et al. (2003). In the model, the technology substitution might induce the economy to accumulate capital more rapidly by curbing consumption at the beginning. I show that if capital is scarce, but resources are abundant, consumption will fall initially, descends to its lower level, and then 
begins the ascend before resource exhaustion. The burning of fossil fuels generates greenhouse gases, and might lead to climate change. Our model does not consider this problem. The catastrophic consequences of climate change might dampen the enthusiasm for the fossil fuel technology, and lead to a more judicious exploitation of the resource. This question is tackled in the third chapter of the thesis. 


\section{References}

[1] Aghion, P, P. Howitt (1998): "Endogenous Growth Theory", MIT Press, Cambridge, MA.

[2] Arrow, K. J. (1962): "The Economic Implications of Learning-by-doing," Review of Economic Studies, 29:155-173.

[3] Barbier, E. (1999): "Endogenous Growth and Natural Resource Scarcity," Environmental and Resource Economics, 14(1): 51-74.

[4] Bretschger, L., S. Smulders (2003): "Sustainability and Substitution of Exhaustible Natural Resources: How Resource Prices Affect Long-Term R\&D Investments," Working Paper 2003.87, Fondazione Eni Enrico Mattei.

[5] Caputo, M.R.(2005): "Foundations of Dynamic Economic Analysis: Optimal Control Theory and Applications," Cambridge University Press, Cambridge

[6] Dasgupta, P. S., G. M. Heal (1974): "The Optimal Depletion of Exhaustible Resources," Review of Economic Studies, 41:3 28.

[7] Groth, C., P. Schou (2002): "Can Non-renewable Resources Alleviate the Knifeedge Character of Endogenous Growth," Oxford Economic Papers, 54:386 411. 
[8] Hartwick, J., N. Van Long, H. Tian (2003): "On the Peaking of Consumption with Exhaustible Resources and Zero Net Investment," Environmental and Resource Economics, 24:235 244.

[9] Hoel, M. (1978): "Resource extraction, substitute production, and monopoly," Journal of Economic Theory, 19(1): 2837.

[10] Hotelling, H. (1931): "The Economics of Exhaustible Resources," Journal of Political Economy, 39:135 175.

[11] Le Van, C., L. Morhaim (2002): "Optimal Growth Models with Bounded or Unbounded Returns: A Unifying Approach," Journal of Economic Theory, 105:158 187.

[12] Lucas, R. E., Jr. (1988): "On the Mechanics of Economic Development," Journal of Monetary Economics, 22:3-42.

[13] Nordhaus, W. D. (1973): "The Allocation of Energy Resources," Brookings Papers on Economic Activity, 4(3): 529576.

[14] Pezzey, J., C. Withagen (1998): "The Rise, Fall and Sustainability of CapitalResource economies," Scandinavian Journal of Economics 100:513 527.

[15] Pitchford, J. D. (1977) "The two stae variable Problems," in Pitchford, J. D, S. J. Turnovsky (1977): "Application of Control Theory to Economic Analysis," North Holland, Amsterdam 101:127 154.

[16] Rebelo, S. (1991): "Long run policy analysis and long run growth," Journal of Political Economy, 99:500 521.

[17] Romer, P. (1986): "Increasing returns and long run growth," Journal of Political Economy, 94:1002 1037. 
[18] Scholz, C. M., G. Ziemes (1999): "Exhaustible Resources, Monopolistic Competition, and Endogenous Growth," Environmental and Resource Economics, 13:169 185 .

[19] Schou, P. (1999): "Endogenous Growth, Non-renewable Resources and Environmental Problems," Ph.D. dissertation, Copenhagen University.

[20] Smulders, S. (2004): "Non-renewable Resources and Economic Growth: Comparing the Classics to New Models of Endogenous Technology and Growth," Lecture notes, Summer School in Resource and Environmental Economics … Dynamic Models in Economics and the Environment.

[21] - (1999): "Endogenous Growth Theory and the Environment," in J. C. J. M. van den Bergh (ed.), Handbook of Environmental and Resource Economics, Edward Elgar Publishing Inc., Cheltenham, UK.

[22] Solow, R. M. (1974): "Intergenerational Equity and Exhaustible Resources," Review of Economic Studies, 4:29 45.

[23] Stiglitz, J. (1974): "Growth with Exhaustible Natural Resources: Efficient and Optimal Growth Paths," Review of Economic Studies, 41:123-137.

[24] Stiglitz, J. E., and P. Dasgupta (1982): "Market structure and resource depletion: A contribution to the theory of intertemporal monopolistic competition," Journal of Economic Theory, 28(1): 128. 164.

[25] Stokey, N. L. (1998): "Are There Limits to Growth?" International Economic Review, 39:1 31. 
[26] Hartwick, J., N. Van Long, H. Tian (2003): "On the Peaking of Consumption with Exhaustible Resources and Zero Net Investment," Environmental and Resource Economics, 24:235 244. 


\section{Appendix 2.A Proof of Lemma 1}

(i) Let $\psi: t \rightarrow \psi(t)=\left(K_{1}(t), x(t), c(t)\right), t \geq \tau$, be the optimal control policy associated with $v(\xi . \eta, \tau)$, and $(X(t \mid \psi), K(t \mid \psi)), t \geq \tau$, be the solution of the system of differential equations (2.3.2), (2.3.3), which is the state of the system at each instant when the control policy $\psi: t \rightarrow \psi(t), t \geq \tau$, is carried out. That is,

$$
\begin{aligned}
\frac{d}{d t} X(t \mid \psi) & =-x(t), \\
X(\tau \mid \psi) & =\xi \\
\frac{d}{d t} K(t \mid \psi) & =A K_{1}^{\alpha}(t)\left(x(t)+b\left(K(t \mid \psi 1)-K_{1}(t)\right)\right)^{1-\alpha}-c(t), \\
K(\tau \mid \psi) & =\eta .
\end{aligned}
$$

Note that because $\psi$ is optimal under $v(\xi, \eta, \tau)$, it is a priori feasible, i.e., the state of the system driven by $\psi$ satisfies the requirement $X(t \mid \psi) \geq 0, K(t \mid \psi)$, and $K_{1}(t) \leq$ $K(t)$, for all $t \geq 0$. Now for any $\lambda>0$, it follows immediately from the linear homogeneity of the differential equation (2.A.1) in the state variable $X(t)$ and the control variable $X(t)$ that

$$
\begin{aligned}
\frac{d}{d t} \lambda X(t \mid \psi) & =-\lambda x(t), \\
\lambda X(\tau \mid \psi) & =\lambda \xi .
\end{aligned}
$$

Similarly, it follows from (2.A.2), the linear homogeneity of the backstop technology, and the linear homogeneity of the technology used in the production of the consumption good that

$$
\begin{aligned}
\frac{d}{d t} \lambda K(t \mid \psi) & =\lambda\left(A K_{1}^{\alpha}(t)\left(x(t)+b\left(K(t \mid \psi)-K_{1}(t)\right)\right)^{1-\alpha}-c(t)\right) \\
& =A\left(\lambda K_{1}(t)\right)^{\alpha}\left(\lambda x(t)+b\left(1 \lambda K(t \mid \psi)-\lambda K_{1}(t)\right)\right)^{1-\alpha}-\lambda c(t), \\
\lambda K(\tau \mid \psi) & =\lambda \eta .
\end{aligned}
$$

I have just shown that if the control policy $\lambda \psi: t \rightarrow \lambda \psi(t)=\left(\lambda K_{1}(t), \lambda x(t), \lambda c(t)\right)$ ,$t \geq \tau$, is carried out, the state of the system at each instant will be given by 
$(\lambda X(t), \lambda K(t)) \geq 0$, and $\lambda K_{1}(t) \leq \lambda K^{\prime}(t)$ for all $t \geq 0$. That is, $\lambda \psi$ is a feasible control policy for the optimization associated with $v(\lambda \xi, \lambda \eta, \tau)$. Thus,

$$
\begin{aligned}
v(\lambda \xi, \lambda \eta, \tau) & \geq \int_{\tau}^{\infty} e^{-\rho t} \frac{(\lambda c(t))^{1-\theta}}{1-\theta} d t \\
& =\lambda^{1-\theta} \int_{\tau}^{\infty} e^{-\rho t} \frac{c(t)^{1-\theta}}{1-\theta} d t \\
& =\lambda^{1-\theta} v(\xi, \eta, \tau) .
\end{aligned}
$$

The argument used to establish (2.A.5) also allows us to assert that

$$
v(\xi, \eta, \tau)=v\left(\frac{1}{\lambda} \lambda \xi, \frac{1}{\lambda} \lambda \eta, \tau\right) \geq\left(\frac{1}{\lambda}\right)^{1-\theta} v(\lambda \xi, \lambda \eta, \tau)
$$

That is,

$$
\lambda^{1-\theta} v(\xi, \eta, \tau) \geq v(\lambda \xi, \lambda \eta, \tau) .
$$

Together, (2.A.5) and (2.A.7) imply that

$$
v(\lambda \xi, \lambda \eta, \lambda \kappa, \tau)=\lambda^{1-\theta} v(\xi, \eta, \kappa, \tau),
$$

i.e., for any given $\tau,(\xi, \eta) \rightarrow v(\xi, \eta, \tau)$ is homogeneous of degree $1-\theta$. This last result also implies the linear homogeneity of the optimal control policy with respect to $(\xi, \eta)$. (i) The concavity of $v(\xi, \varphi, \tau)$ follows from the fact that the Cobb-Douglas production function is concave in the capital and energy inputs. 


\section{Appendix 2.B The derivation of the HJB equation and the adjoint equations}

\section{Derivation of the Hamilton-Jacobi-Bellman equa- tion and the adjoint equations}

\section{The Hamilton-Jacobi-Bellman equation}

Let $t \rightarrow\left(K_{1}(t), x(t), c(l)\right), t \geq \tau$, be an admissible control policy. Now consider an infinitesimal time interval $(\tau, \tau+\Delta \mathrm{t})$. If we carry out this control policy during the infinitesimal time interval $[\tau, \tau+\Delta t)$, and then switch to the optimal control policy from time $\tau+\Delta t$ on, then the discounted utilities obtained under this action are given by

$$
e^{-\rho \tau} u(c(\tau)) \Delta \mathrm{t}+o(\Delta \mathrm{t})+v(X(\tau+\Delta \mathrm{t}), K(\tau+\Delta \mathrm{t}), \tau+\Delta \mathrm{t})
$$

Because such an action is not necessarily optimal, we must have

$$
e^{-\rho \tau} u(c(\tau)) \Delta \mathrm{t}+o(\Delta \mathrm{t})+v(X(\tau+\Delta \mathrm{t}), K(\tau+\Delta \mathrm{t}), \tau+\Delta \mathrm{t}) \leq v(\xi, \eta, \tau)
$$

with equality holding if $t \rightarrow\left(K_{1}(t), x(t), c(t)\right), t \geq \tau$, is optimal during the infinitesimal time interval $[\tau, \tau+\Delta t)$.

Let us rewrite (2.B.1) as

$$
e^{-\rho \tau} u(c(\tau)) \Delta \mathrm{t}+o(\Delta \mathrm{t})+v(X(\tau+\Delta \mathrm{t}), K(\tau+\Delta \mathrm{t}), \tau+\Delta \mathrm{t})-v(\xi, \eta, \tau) \leq 0
$$

with equality holding if $t \rightarrow\left(K_{1}(t), x(t), c(t)\right), t \geq \tau$, is optimal during the infinitesimal time interval $[\tau, \tau+\Delta \mathrm{t})$. 
Now dividing (2.B.2) by $\Delta t$, and then letting $\Delta t \rightarrow 0$, we obtain

$$
\begin{aligned}
& e^{-\rho \tau} u(c(\tau))-D_{1} v(\xi, \eta, \tau) x(\tau)+ \\
& D_{2} v(\xi, \eta, \tau)\left(A K_{1}^{\alpha}(\tau)\left(x(\tau)+b\left(K(\tau)-K_{1}(\tau)\right)\right)^{1-\alpha}-c(\tau)\right)+ \\
& D_{3} v(\xi, \eta, \tau) k \leq 0
\end{aligned}
$$

with equality holding if $\left(K_{1}(\tau), x(\tau), c(\tau)\right)$ is optimal at time $\tau$. Here $D_{1} v(\xi, \eta, \tau)$, $D_{2} v(\xi, \eta, \tau)$, and $D_{3} v(\xi, \eta, \tau)$ denote, respectively, the partial derivatives of $v(\xi, \eta, \tau)$ with respect to $\xi, \eta$, and $\tau$.

We represent this result more compactly under the following form, known as the Hamilton-Jacobi-Bellman equation for the model of exhaustible resources, technology substitution, and endogenous growth:

$$
\begin{aligned}
0=\max _{\left(K_{1}, x, c\right)}\left(e^{-\rho \tau} u(c)-D_{1} v(\xi, \eta, \tau) x+\right. \\
\left.\quad D_{2} v(\xi, \eta, \tau)\left(A K_{1}^{\alpha}\left(x+b\left(\eta-K_{1}\right)\right)^{1-\alpha}-c\right)+D_{3} v(\xi, \eta, \tau)\right),
\end{aligned}
$$

and the optimal controls are the values of $K_{1}, x$, and $c$ that solves the maximization problem in (2.B.4).

Setting $(\xi, \eta, \tau)=(X, K, t)$, with $(X, K, t)$ being an arbitrary state of the system, in (2.B.4), we obtain the following general version of the Hamilton-Jacobi-Bellman equation that the optimal value must satisfy when at some arbitrary time $t$, the remaining stock of oil is $X$ and the stock of capital is $K$. 


$$
\begin{aligned}
0=\max _{\left(K_{1}, x, c\right)} & \left(e^{-\rho \tau} u(c)-D_{1} v(X, K, t) x+\right. \\
& \left.D_{2} v(X, K, t)\left(A K_{1}^{\alpha}\left(x+b\left(K-K_{1}\right)\right)^{1-\alpha}-c\right)+D_{3} v(X, K, t)\right),
\end{aligned}
$$

and the values of $K_{1}, x, t$ that solves the maximization problem in (2.B.4) constitute the optimal capital allocation to the consumption good sector, the optimal oil extraction rate, and the optimal consumption when the system is in state $(X, K, t)$.

\section{The adjoint equations}

Suppose that we fix $(\xi, \eta, \tau)$ momentarily, and then let $\left(K_{1}(\xi, \eta, \tau), x(\xi, \eta, \tau), c(\xi, \eta, \tau)\right)$ be the value of $\left(K_{1}, x, c\right)$ that solves the maximization problem in (2.B.4). Next, consider an arbitrary state $(X, K, t)$ for the system, and then let

$$
\begin{aligned}
& H(X, K, t)=e^{-\rho t} u(c(\xi, \eta, \tau))-D_{1} v(X, K, t) x(\xi, \eta, \tau)+ \\
& D_{2} v(X, K, t)\left(A K_{1}^{\alpha}(\xi, \eta, \tau)\left(x(\xi, \eta, \tau)+b\left(K-K_{1}(\xi, \eta, \tau)\right)\right)^{1-\alpha}-c(\xi, \eta, \tau)\right)+ \\
& D_{3} v(X, K, t) .
\end{aligned}
$$

As defined, $H(X, K, t) \leq 0$ for all $(X, K, t)$, with equality holding if $(X, K, t)=$ $(\xi, \eta, \tau)$. That is, the map $H:(X, K, t) \rightarrow H(X, K, t)$ is always less than or equal to 0 , and it achieves a global maximum equal to 0 when $(X, K, t)=(\xi, \eta, \tau)$. The following first-order conditions characterize this maximum:

$$
\begin{aligned}
& D_{1} H(X, K, t)=-D_{11} v(X, K, t) x(\xi, \eta, \tau)+ \\
& D_{21} v(X, K, t)\left(A K_{1}^{\alpha}(\xi, \eta, \tau)\left(x(\xi, \eta, \tau)+b\left(K-K_{1}(\xi, \eta, \tau)\right)\right)^{1-\alpha}-c(\xi, \eta, \tau)\right)+ \\
& D_{31} v(X, K, t)=0 .
\end{aligned}
$$




$$
\begin{aligned}
& D_{2} H(X, K, t)=-D_{12} v(X, K, t) x(\xi, \eta . \tau)+ \\
& D_{22} v(X, K, t)\left(A K_{1}^{\alpha}(\xi, \eta, \tau)\left(x(\xi, \eta, \tau)+b\left(K-K_{1}(\xi, \eta, \tau)\right)\right)^{1-\alpha}-c(\xi, \eta, \tau)\right)+ \\
& D_{2} v(X, K, t)\left(\Lambda K_{1}^{\alpha}(\xi, \eta, \tau)\left(x(\xi, \eta, \tau)+b\left(K-K_{1}(\xi, \eta, \tau)\right)\right)^{-\alpha}(1-\alpha) b\right)+ \\
& D_{32} v(X, K, t)=0
\end{aligned}
$$

When (2.B. 7$)$ and (2.B.8) are evaluated at $(X, K, t)=(\xi, \eta, \tau)$, they become, respectively,

$$
\begin{aligned}
& D_{1} H(\xi, \eta, \tau)=-D_{11} v(\xi, \eta, \tau) x(\xi, \eta, \tau)+ \\
& D_{21} v(\xi, \eta, \tau)\left(A K_{1}^{\alpha}(\xi, \eta, \tau)\left(x(\xi, \eta, \tau)+b\left(\eta-K_{1}(\xi, \eta, \tau)\right)\right)^{1-\alpha}-c(\xi, \eta, \tau)\right)+ \\
& D_{31} v(\xi, \eta, \tau)=0, \\
& D_{2} H(\xi, \eta, \tau)=-D_{12} v(\xi, \eta, \tau) x(\xi, \eta, \tau)+ \\
& D_{22} v(\xi, \eta, \tau)\left(A K_{1}^{\alpha}(\xi, \eta, \tau)\left(x(\xi, \eta, \tau)+b\left(\eta-K_{1}(\xi, \eta, \tau)\right)\right)^{1-\alpha}-c(\xi, \eta, \tau)\right)+ \\
& D_{2} v(\xi, \eta, \tau)\left(A K_{1}^{\alpha}(\xi, \eta, \tau)\left(x(\xi, \eta, \tau)+b\left(\eta-K_{1}(\xi, \eta, \tau)\right)\right)^{-\alpha}(1-\alpha) b\right)+ \\
& D_{32} v(\xi, \eta, \tau)=0 .
\end{aligned}
$$

Let us now apply (2.B.4), (2.B.9), and (2.B.10) to the original problem faced by the central planner. To this end, let $\left(K_{1}^{*}(t), x^{*}(t), c^{*}(t)\right), t \geq 0$, be the solution of the problem constituted by (2.2.12) -(2.2.14). Under the optimal controls, the motion of the system is governed by the following differential equations:

$$
\frac{d X^{*}}{d t}=-x^{*}(t), X^{*}(0)>0 \text { isgiven }
$$




$$
\frac{d K^{*}}{d t}=A\left(K_{1}^{*}(t)\right)^{\alpha}\left(x^{*}(t)+b\left(K^{*}(t)-K_{1}^{*}(t)\right)\right)^{1-\alpha}-c^{*}(t), K^{*}(0)>0 \text { isgiven }
$$

Apply (2.B.9) at a point $(\xi, \eta, \tau)=\left(X^{*}(t), K^{*}(t), t\right)$ along the optimal trajectory, we obtain

$$
\begin{aligned}
& D_{1} H\left(X^{*}(t), K^{*}(t), t\right)=-D_{11} v\left(X^{*}(t), K^{*}(t), t\right) x\left({ }^{*} t\right)+ \\
& D_{21} v\left(X^{*}(t), K^{*}(t), t\right)\left(A\left(K_{1}^{*}(t)\right)^{\alpha}\left(x^{*}(t)+b\left(K^{*}(t)-K_{1}^{*}(t)\right)\right)^{1-\alpha}-c^{*}(t)\right)+ \\
& D_{31} v\left(X^{*}(t), K^{*}(t), t\right)=0 .
\end{aligned}
$$

Let

$$
\phi(t)=D_{1} v\left(X^{*}(t), K^{*}(t), t\right)
$$

be the shadow price of oil at a point in time $t$ along the optimal trajectory. The motion of $\phi(t)$ is governed by the following differential equation:

$$
\begin{gathered}
\frac{d \phi}{d t}=D_{11} v\left(X^{*}(t), K^{*}(t), t\right) \frac{d X^{*}}{d t}+D_{12} v\left(X^{*}(t), K^{*}(t), t\right) \frac{d K^{*}}{d t}+D_{13} v\left(X^{*}(t), K^{*}(t), t\right) \\
=-D_{11} v\left(X^{*}(t), K^{*}(t), t\right) x\left(^{*} t\right)+ \\
D_{21} v\left(X^{*}(t), K^{*}(t), t\right)\left(A\left(K_{1}^{*}(t)\right)^{\alpha}\left(x^{*}(t)+b\left(K^{*}(t)-K_{1}^{*}(t)\right)\right)^{1-\alpha}-c^{*}(t)\right)+ \\
D_{31} v\left(X^{*}(t), K^{*}(t), t\right)=0
\end{gathered}
$$

where the last equality is due to (2.B.13).

Apply (2.B.10) at a point $(\xi, \eta, \tau)=\left(X^{*}(t), K^{*}(t), t\right)$ along the optimal trajectory, we obtain 


$$
\begin{array}{r}
D_{2} H\left(X^{*}(l), K^{*}(t), l\right)=-D_{12} v\left(X^{*}(l), K^{*}(t), t\right) x\left({ }^{*} t\right)+ \\
D_{22} v\left(X^{*}(t), K^{*}(t), t\right)\left(A\left(K_{1}^{*}(t)\right)^{\alpha}\left(x^{*}(t)+b\left(K^{*}(t)-K_{1}^{*}(t)\right)\right)^{1-\alpha}-c^{*}(t)\right)+ \\
D_{2} v\left(X^{*}(t), K^{*}(t), t\right)\left(A\left(K_{1}^{*}(t)\right)^{\alpha}\left(x^{*}(t)+b\left(K^{*}(t)-K_{1}^{*}(t)\right)\right)^{-\alpha}(1-\alpha) b\right)+ \\
D_{32} v\left(X^{*}(t), K^{*}(t), t\right)=0 .
\end{array}
$$

Let

$$
\varphi(t)=D_{2} v\left(X^{*}(t), K^{*}(t), t\right)
$$

be the shadow price of capital at a point in time $t$ along the optimal trajectory. The motion of $\varphi(t)$ is governed by the following differential equation:

$$
\begin{aligned}
\frac{\mathrm{d} \varphi}{\mathrm{d} t} & =-D_{21} v\left(X^{*}(t), K^{*}(t), t\right) x\left({ }^{*} t\right)+ \\
& D_{22} v\left(X^{*}(t), K^{* *}(t), t\right)\left(A\left(K_{1}^{*}(t)\right)^{\alpha}\left(x^{*}(t)+b\left(K^{*}(t)-K_{1}^{*}(t)\right)\right)^{1-\alpha}-c^{*}(t)\right)+ \\
& D_{32} v\left(X^{*}(t), K^{* *}(t), t\right) \\
& =-D_{2} v\left(X^{*}(t), K^{*}(t), t\right)\left(A\left(K_{1}^{*}(t)\right)^{\alpha}\left(x^{*}(t)+b\left(K^{*}(t)-K_{1}^{*}(t)\right)\right)^{-\alpha}(1-\alpha) b\right) \\
& =-\varphi(t)\left(A\left(K_{1}^{*}(t)\right)^{\alpha}\left(x^{*}(t)+b\left(K^{*}(t)-K_{1}^{*}(t)\right)\right)^{-\alpha}(1-\alpha) b\right),
\end{aligned}
$$

where the second equality in (2.B.18) is due to (2.B.16), and the third equality follows from the definition of $\varphi(t)$.

The differential equations (2.B.15) and (2.B.18) can be expressed under the following forms:

$$
\frac{d \phi}{d t}=-D_{1} \mathscr{H}\left(X^{*}(t), K^{*}(t), K_{1}^{*}(t), x^{*}(t), c^{*}(t), \phi(t), \varphi(t), t\right)
$$




$$
\frac{d \varphi}{d t}=-D_{2} \mathcal{H}\left(X^{*}(t), K^{*}(t), K_{1}^{*}(t), x^{*}(t), c^{*}(t), \phi(t), \varphi(t), t\right),
$$

where the Hamiltonian $\mathscr{H}$ is defined as follows:

$$
\begin{aligned}
\mathscr{H}\left(X, K, K_{1}, x, c, \phi, \varphi, t\right) & =e^{-\rho t} u(c)+\phi \frac{d X}{d t}+\varphi \frac{d K}{d t} \\
& =e^{-\rho t} u(c)-\phi x+\varphi\left(A K_{1}^{\alpha}\left(x+b\left(K-K_{1}\right)\right)^{1-\alpha}-c\right) .
\end{aligned}
$$

As for the Hamilton-Jacobi-Bellman equation (2.B.4) at a point $\left(X^{*}(t), K^{*}(t), t\right)$ along the optimal trahectory, it can be expressed under the following form:

$$
\begin{aligned}
0 & =\max _{\left(K_{1}, x, c\right)} \mathscr{H}\left(X^{*}(t), K^{*}(t), K_{1}, x, c, \phi(t), \varphi(t), t\right)+D_{3} v\left(X^{*}(t), K^{*}(t), t\right) . \\
\mathrm{al}^{0} & =\max _{\left(K_{1}, x, c\right)} \mathscr{H}\left(X^{*}(t), K^{*}(t), K_{1}, x, c, \phi(t), \varphi(t), t\right)+D_{3} v\left(X^{*}(t), K^{*}(t), t\right) .
\end{aligned}
$$

maximization problem in (2.B.22).

\section{Appendix 2.C The closed-form expression for the capital stock before oil exhaustion}

In this appendix, I solve the differential equation (2.5.33), which governs the motion of the stock of capital before oil exhaustion. This differential equation is reproduced here as

$$
\begin{aligned}
& \frac{d K^{*}}{d t}-A^{\frac{1}{\alpha}}\left(\frac{(1-\alpha)(-b \phi t+\zeta)}{\phi}\right)^{\frac{1-\alpha}{\alpha}} K^{*}(t)=-\left(e^{\rho t}(-b \phi t+\zeta)\right)^{-\frac{1}{\theta}} \\
& K^{*}(0)>0 \text { is given. }
\end{aligned}
$$

Here $\zeta=\varphi(0)$ is the constant of integration, which is also the shadow price of capital at time $t=0$. 
Note that (2.C.1) is a first-order linear differential equation in $K^{*}(t)$, and can be solved by using the following integrating factor

$$
\begin{aligned}
& e^{\int-A^{\frac{1}{\alpha}}(1-\alpha)^{1+\frac{1}{\alpha}} \phi^{1+\alpha}}(\zeta-b t \phi)^{1+\frac{1}{\alpha}} d t=e^{A^{1 \frac{1}{\alpha}(1-\alpha)^{-1+\frac{1}{\alpha}} \frac{\alpha \phi}{b}-1 / \alpha 2}(\zeta-b t \phi)^{\frac{1}{\alpha}}} . \\
& e^{\int-A \frac{1}{\alpha}(1-\alpha)^{-1+\frac{1}{\alpha} \phi} \frac{-1+\alpha}{\alpha}(\zeta-b t \phi)^{-1+\frac{1}{\alpha}} d t} \\
& =e^{\frac{A^{\frac{1}{\alpha}(1-\alpha)}-1+\frac{1}{\alpha} \alpha \phi^{-1 / \alpha}(\zeta-b t \phi) \frac{1}{\alpha}}{b}} \text {. } \\
& =e^{\frac{A^{\frac{1}{\alpha}}(1-\alpha)^{-1+\frac{1}{\alpha}} \alpha \phi^{-1 / \alpha}(\zeta-b t \phi)^{\frac{1}{\alpha}}}{b}}
\end{aligned}
$$

Using the integrating factor (2.C.2), just computed, I can rewrite (2.C.1) as

$$
\frac{d}{d t}\left(K^{*}(t) e^{\frac{\left.A^{\frac{1}{\alpha}(1-\alpha)}\right)^{-1+\frac{1}{\alpha}} \alpha\left(-b t+\frac{\zeta}{\phi}\right)^{\frac{1}{\alpha}}}{b}}\right)=-e^{-\frac{t \rho}{\theta}}(-b t \phi+\zeta)^{-1 / \theta} e^{\frac{A^{\frac{1}{\alpha}(1-\alpha)}-1+\frac{1}{\alpha} \alpha\left(-b t+\frac{\zeta}{\phi}\right)^{\frac{1}{\alpha}}}{b}}
$$

Integrating (2.C.3), we obtain

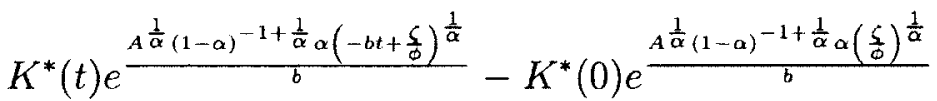

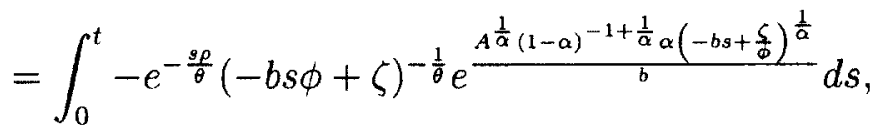

which can be rewritten as

$$
\begin{aligned}
K^{*}(t) & =K^{*}(0) e^{\frac{A^{\frac{1}{\alpha}(1-\alpha)^{-1+\frac{1}{\alpha} \alpha}\left(\left(\frac{\zeta}{\phi}\right)^{\frac{1}{\alpha}}-\left(-b t+\frac{\zeta}{\phi}\right)^{\frac{1}{\alpha}}\right)}}{b}} \\
& -e^{-\frac{A^{\frac{1}{\alpha}(1-\alpha)}+1+\frac{1}{\alpha} \alpha\left(-b t+\frac{\zeta}{\phi}\right)^{\frac{1}{\alpha}}}{b}} \int_{0}^{t} e^{-\frac{s \rho}{\theta}+\frac{A^{\frac{1}{\alpha}(1-\alpha)} 1+\frac{1}{\alpha} \alpha\left(-b s+\frac{\zeta}{\phi}\right)^{\frac{1}{\alpha}}}{b}}(\zeta-b s \phi)^{-\frac{1}{\theta}} d s .
\end{aligned}
$$




\section{Essay 3}

\section{The Low-Probability}

\section{High-Consequence Events In Environmental Economics}

\subsection{Introduction}

Scientists believe that human activities are mainly responsible for the change in climate since the industrial revolution. Concerns are about the emission of carbon dioxide $\left(\mathrm{CO}_{2}\right)$, methane $\left(\mathrm{CH}_{4}\right)$, nitrous oxide $\left(\mathrm{N}_{2} \mathrm{O}\right)$, water vapor $\left(\mathrm{H}_{2} \mathrm{O}\right)$, etc., the major greenhouse gases (GHGs) that are responsible for climate change. The International Panel of Climate Change (IPCC) concluded in 2001 that there is new and stronger evidence that most of the warming observed over at least the past 50 years is attributable to human activities (IPCC-2001). In a later report (IPCC-2007), the agency stated that "continued GHG emissions at or above current rates would cause further warming and induce many changes in the global climate system during the $21^{s t}$ century that would very likely be larger than those observed during the $20^{t h}$ 
century." In fact, the share of fossil fuels in current energy primary use amounts to $80 \%$ globally, and energy consumption will increase by $44 \%$ globally in 2030 (EIA 2006). Some of the global impacts may amplify the warming further by triggering the release of additional methane gas trapped in an area, such as Northern Siberia, which may become another threat in the coming years. A study published in the September 7, 2006 issue of Nature reported that lakes in the permafrost zone of Northern Siberia are now emitting much more methane into the atmosphere than previously thought. With the increase of temperature, induced by a high volume of greenhouse gases in the atmosphere, a massive release of methane from the melting permafrost (Harvey and Huang, 1995) would lead to rapid warming worldwide. Global climate models project the strongest future warming in the high latitudes, with some models predicting a 7 to 8 degree Celsius $\left({ }^{\circ} \mathrm{C}\right)$ warming over land in these regions by the end of the $21^{\text {st }}$ century (IPCC 2007).

The negative consequences of a change in the climate are estimated by different sources to be extremely high, while the probability of the occurrence of these events is very low. In other words, the climate change may lead to catastrophic events which can be worse than the disasters that we have already experienced and recorded in the recent past, such as hurricanes, forest fires, drought, floods, species extinction, irreversibility, and destruction of some ecosystems. The consequences may be considered as a threat to human lives directly by intense flooding, large forest fires, and unknown emerging diseases related to ecosystem and abrupt temperature changes. These impacts are long-term and persistent. Uncertainties and risks in the economic impacts are global. There is a serious risk of major irreversible change with non-marginal economic effects, at least at the human life time scale.

Economists have approached the global environmental issues from different perspectives and traditions. A large number of instruments to regulate pollution activ- 
ities have been proposed: Pigouvian taxes, quotas, subsidies for pollution reduction, emissions permits (Dales, 1968a, 1968b), deposit refunds systems (Bohm, 1981), assignment of legal liabilities (Segerson, 1995), Boyer and Laffont (1996,1997), Gupta, van Houtven and Cropper (1996)). Debates about which instruments to use have increased in the last two decades. The discussion has taken place within different paradigms related to the social planner's social welfare maximization (Cropper and Oates (1992), Segerson (1996), and Lewis (1997)), or self interest as in the BuchananTullock political economy traditions (Dewes (1983), Coelho (1976), Boyer (1979), Noll (1983), Hahn (1990), and Hahn and McGartland (1989)), and others. Recently, the political economy of environmental issues has been analyzed from the perspective of mechanism design theory and asymmetric information; see, for example, Dasgupta, Hammond and Maskin (1980), Baron (1985a), Spulber (1998), Laffont (1994), Boyer and Laffont (1999), Lewis (1997), Ulph (1998), Aidt and Dutta (2004), and Sheriff (2008). The political economy strand has also proposed some voting models which deal with environmental issues in national or international contexts. The innovation in these recent studies is the modeling of the influence of special-interest groups on the general public and the politicians in the design of policies and the choice of instruments.

Other researchers concentrated on the possibility of irreversibility in the natural environment and its consequences on social welfare (Cropper (1976), Clark and Reed (1994), and Tsur and Zimel (2006, 2007)). Threats of environmental catastrophes triggered by human activities were also analyzed by researchers, such as Cropper (1976) and Arronson et al. (1998), who studied events of nuclear accidents. Examples of studies related to events of global warming calamities are presented in Fisher et al (2003), Hayohe et al. (2004), IPCC (2001, 2007), and Tsur and Zimel (1998). All these studies considered a utility function that depends on both consumption 
and pollution. Once the pollution threshold level is surpassed, with certain probability, consumption drops to zero. Examples and discussions related to non-convex phenomena in ecological systems are addressed by Dasgupta and Mäler (2003) and Mäler (2000). An interesting example of the use of bifurcation theory in economics is due to Wagener (2003), who applied it to the lake system studied by Dasgupta and Mäler(2003), and found multiple equilibria in an ecologic-economic model of pollutants in a lake system (Brock et al. (1999), Brock and Starrett (2003), Dechert and O'Donnell (2006)).

In the model of economic growth of the Solow tradition, labor and capital are the only two factors of production. Growth in this type of model is driven by the accumulation of capital and by exogenous technical progress. Recent growth theories endogenize technological progress for example through an increase in the stock of knowledge (Romer, 1991) or through the accumulation of human capital (Lucas 1988, 1993). These models allow for exponential growth, and the environment as a factor of production is almost absent in these models. Nature, if it ever appears in a macroeconomic model, is a fixed, indestructible factor of production, called land following Ricardo. In an economic model of climate change, it is not possible to ignore the role of energy and the environment.

Human existence and activities benefit from the natural environment in many ways. The environment is the source of many varied renewable resources, such as fish, forests and plants, clean air, and water systems with a variety of aquatic animals and plants, etc., as well as the source of many exhaustible resources, such as oil and gas. The natural environment also serves as a sink for human-produced wastesexternalities in the atmosphere, under the ground, on land, and in water systems. The environment has the capacity to assimilate most of the wastes and regenerate itself. However, this regenerating capacity is not infinite. The first law of thermody- 
namics states that matter or energy can neither be created nor destroyed. Mass of materials entering the economic system from the environment either accumulates in the economic system itself (e.g. metals) or is returned to environment as waste (e.g. tailings). Those wastes may negatively affect (depreciate) the environmental assets. It is thus legitimate to consider the environment as a dynamic system which consists of one sub-system that is non-renewable (minerals, coal, oil, etc.) and one subsystem that is renewable. The renewable subsystem of the environment also known as ecosystems-provides visible products, such as food, fibres, fuel, and fresh water. Ecosystems also house a genetic library, preserve and regenerate soil, operate the hydrological cycle, etc. In the economic literature, these two sub-systems are referred to as natural capital (Dasgupta (2010), England(2000)).

In this paper, I set up a model that links the burning of fossil fuels to climate change through the accumulation of greenhouse gases in the atmosphere. In this model, fossil fuels are extracted from the non-renewable sub-system of natural capital. I shall not attempt to model the renewable sub-system of natural capital in all its comprehensive splendor. Instead, I use the stock of greenhouse gases in the atmosphere as a proxy for the renewable sub-system of natural capital. Also, in what follows, I interchangeably use oil as a generic term for fossil fuels.

The natural capital that is embodied in the non-renewable sub-system inevitably declines with extraction. The regenerating capacity of the renewable sub-system might decline because of the extracting activities and adverse effects caused by the unrestrained use of it as a sink for wastes, especially the emissions of carbon dioxide into the atmosphere from the burning of fossil fuels. In this paper, economic growth is linked to its adverse consequences on the environment. The model I develop is a stochastic model of economic growth in which the burning of fossil fuels raises the stock of greenhouse gases in the atmosphere, which might lead to climate change, and 
climate change in turn leads to catastrophic events, such as a rise in the sea level, which might flood important areas of different countries, or a severe drought, which might cause mass migration of farmers to cities, or the melting of the permafrost, which triggers a massive release of the greenhouse gases trapped in this region of the earth. Such an event, when it occurs, causes massive damages to the stock of manmade capital as well as the stock of natural capital, and raises the stock of greenhouse gases in the atmosphere. To keep the model tractable, I follow the researchers in this field and assume that once a catastrophe occurs, there is irreversible damage to the environment which reduces consumption permanently to such a low level that I take to be zero. To model the low probabilities with which such a catastrophic event occurs and its arrival time, I use the Poisson process.

In the present model, a consumption good is produced, using three essential inputs labor, capital, and a composite energy input. The composite energy input is generated by burning fossil fuels, by exploiting a backstop, or by using both technologies. Although oil can be extracted at negligible cost, its stock is limited. The backstop, on the other hand, can produce a perpetual flow of energy. However, energy produced from the backstop requires capital solar collectors, wind turbines. As oil resources dwindle, the backstop must be brought into use to prevent a drastic reduction in consumption. Furthermore, in the context of climate change, the substitution of renewable energy for fossil fuels also reduces greenhouse gas emissions, and this lowers the probability of catastrophic events. The substitution of renewable energy for energy generated by burning fossil fuels is thus an important economic issue.

The paper is organized as follows. In Section 2, the stochastic growth model is presented. In the stochastic growth model, a catastrophe might occur only once. The arrival time of the catastrophe follows a Poisson process, which depends on the stock of greenhouse gases in the atmosphere. Furthermore, when the catastrophe occurs, there 
is irreversible damage to the environment, and consumption is permanently reduced to zero. The mathematical machinery I use for modeling and analyzing a stochastic control problem in which the state of the system might experience a discrete jump at any instant according a Poisson process is based upon Dreyfus (1965). In Section 3 , I use the dynamic programming formalism to derive the Hamilton-Jacobi Bellman equation for the stochastic growth problem. The derivations of the Hamilton-JacobiBellman equation and the differential equations that govern the motion of the shadow prices of oil, capital, natural capital, and greenhouse gases are given in Appendix A of the paper. In deriving theses equations, I have used the heuristic arguments based on the dynamic programming formalism, as developed by Bekovits (1974). Section 4 contains the first-order conditions -obtained by maximizing the Hamiltonian- that characterize the optimal solution at each point in time. In Section 5, I demonstrate that the value of the current Hamiltonian at each point in time along the optimal trajectory represents the green GDP at that instant.

\subsection{The model}

In the model, time is continuous and denoted by $t, t \geq 0$. The economy produces a consumption good, using three inputs capital, labor, and a composite energy input. The composite energy input is generated by combining energy from two sources - fossil fuels and a backstop. The burning of fossil fuels generates greenhouse gases, and might lead to catastrophic climate change. The backstop, which produces renewable energy, is non-polluting. In what follows, I also refer to fossil fuels as oil. 


\subsubsection{Preferences}

Let $u(c)$ denote the instantaneous utility of consumption. I assume that

$$
u(c)=\frac{c^{1-\theta}}{1-\theta}
$$

where $\theta, 0<\theta<1$, is a parameter, which is the inverse of the inter-temporal elasticity of substitution.

\subsubsection{The energy technologies}

The backstop produces renewable energy, using only capital. I assume that if $K_{0}$ is the capital input used in the backstop sector, then the amount of renewable energy measured in Btu is equal to $\beta K_{0}$, where $0<\beta \leq 1$, is a parameter. That is, one unit of capital used in the backstop sector allows the economy to harness $\beta$ Btu from the Sun.

If $x$ is the amount of fossil fuel expressed in Btus burned at an instant, and $K_{0}$ is the capital input used in the backstop sector, then the composite energy input generated is assumed to be given by

$$
q=x+\beta K_{0}
$$

According to (3.2.2), energy obtained by burning fossil fuels and renewable energy are perfect substitutes for generating the composite energy input.

\subsubsection{The consumption good technology}

The consumption good is produced according to the following technology:

$$
Y=A F\left(K_{1}, q, L\right)
$$


where $Y$ represents output, $K_{1}$ the capital input, $q$ the composite energy input, and $L$ the labor input. Also, $A$ is the technological level. The population is assumed to be constant and normalized to 1 . In what follows, I assume that $F\left(K_{1}, q, 1\right)$ has the Cobb-Douglas form:

$$
F\left(K_{1}, q, 1\right)=K_{1}^{\alpha} q^{1-\alpha}
$$

where $\alpha, 0<\alpha<1$, is a parameter. Under this assumption, the technology for producing the consumption good becomes

$$
Y=A F\left(K_{1}, q, 1\right)=A K_{1}^{\alpha} q^{1-\alpha}
$$

which implies that with a fixed labor input there is constant return to scale of the other two factors - capital and energy. The production function represented by (3.2.5) is thus the technology of the AK model of endogenous growth à la Arrow (1962) and Romer (1986).

Let $K$ be the capital stock of the economy at any instant. The allocation of capital between the backstop sector and the consumption good sector at that instant satisfies the following identity:

$$
K=K_{0}+K_{1}
$$

The collapse of the two capital stocks to one state variable is due to the fact that both capitals $K_{0}$ and $K_{1}$ are considered as of the same nature and measured with the same units. For more discussion on this subject, see Pitchford and Turnovsky (1977).

\subsubsection{Catastrophe, its probabilities and consequences}

Consider an infinitesimal time interval $[t, t+\Delta t)$. The probability that a catastrophe occurs during this time interval is assumed to depend on the stock of greenhouse gases at time $l$ in the atmosphere and is given by

$$
\lambda H(t) \Delta t+o(\Delta t)
$$


where $\lambda>0$ is a parameter. In (3.2.7),o( $\Delta t)$ is a term such that $\lim _{\Delta t \rightarrow 0} \frac{o(\Delta t)}{\Delta t}=0$. The probability that no catastrophe occurs during the infinitesimal time interval $[t, t+\Delta t)$ is

$$
1-\lambda H(t) \Delta t+o(\Delta t)
$$

The probability of two or more catastrophes occurring during the time interval $[t, t+\Delta t)$ is $o(\Delta t)$.

To keep the model tractable, I shall assume that once a catastrophe occurs, the damage to the environment is so severe that only a very low level of consumption can be sustained for a long time after that. More specifically, I assume that once a catastrophe has occurred, consumption is forever reduced to 0 .

Let $P(t)$ denote the probability that no catastrophe has occurred by time $t>0$. If no catastrophe has occurred by time $t$, then the probability that no catastrophe occurs by time $t+h$ is given by

$$
P(t+h)=P(t)(1-\lambda H(t) h+o(h)),
$$

which can be rewritten as

$$
P(t+h)-P(t)=P(t)(-\lambda H(t) h+o(h)) .
$$

Dividing (3.2.8) by $h$, and then letting $h \rightarrow 0$, WE obtain

$$
\frac{d P}{d t}=-\lambda H(t) P(t)
$$

The solution of the differential equation (3.2.9), with the initial condition $P(0)=1$, is

$$
P(t)=e^{-\lambda \int_{0}^{t} H(s) d s}
$$

The probability that a catastrophe occurs by time $t$ is then given by

$$
F(t)=1-P(t)=1-e^{-\lambda \int_{0}^{t} H(s) d s} .
$$


The density of $F$ is

$$
f(t)=F^{\prime}(t)=\lambda H(t) e^{-\lambda \int_{0}^{t} H(s) d s} .
$$

\subsubsection{Formal statement of the optimal growth problem with two energy sectors and possible climate change}

At each time $t$, the state of the system is represented by the list $(X(t), K(t), H(t))$, where $X(t), K(t)$, and $H(t)$ denote, respectively, the remaining stock of fossil fuels, the stock of capital, and the stock of greenhouse gases in the atmosphere. The central planner has to choose

(i) an allocation of the capital stock $K(t)$ between the backstop sector $\left(K_{0}(t)\right)$ and the consumption good sector $\left(K_{1}(t)\right)$, with $K_{0}(t)+K_{1}(t)=K(t)$,

(ii) $c(t)$, the consumption,

(iii) $x(t)$, the oil extraction rate, and

(iv) $k(t)$, the capital investment

Under such a policy, the motion of the remaining stock of oil is governed by the following differential equation:

$$
\begin{aligned}
& \frac{d X}{d t}=-x(t), \\
& X(0)=X_{0}>0 \quad \text { is given. }
\end{aligned}
$$

The motion of the stock of capital is governed by the following differential equation:

$$
\begin{aligned}
\frac{d K}{d t} & =k(l), \\
K(0) & =K_{0}>0 \quad \text { is given. }
\end{aligned}
$$


and the evolution of the stock of greenhouse gases in the atmosphere is governed by the following differential equation:

$$
\begin{aligned}
& \frac{d H}{d t}=x(t)-\delta(H(t)-\underline{H}), \\
& H(0)=H_{0} \geq \underline{H} \text { is given. }
\end{aligned}
$$

In $(3.2 .15), \delta>0$ is a parameter, which represents the rate at which greenhouse gases decay, and $\underline{H}>0$ is the natural level of greenhouse gases in the atmosphere. Without any loss of generality, I shall from now on assume that $\underline{H}=0$.

I have assumed that if a catastrophe has occurred, the consumption at each time after that is reduced to 0 forever, and the problem ends. Thus, consider an instant at which no catastrophe has occurred. Suppose that the central planner carries out a policy $t \rightarrow\left(K_{0}(t), K_{1}(t), x(t), c(t)\right), t \geq 0$. Under such a policy, the expected discounted value of the stream of social welfare obtained is given by

$$
\begin{aligned}
\int_{0}^{\infty}\left(\int_{0}^{t} e^{-\rho s} u(c(s)) d s\right) f(t) d t & =\int_{0}^{\infty} e^{-\rho t} u(c(s)) P(t) d t \\
& =\int_{0}^{\infty}\left(e^{-\rho t} u(c(s)) e^{-\lambda \int_{0}^{t} I(s) d s}\right) d t
\end{aligned}
$$

where the expression on the right side of the first equality in (3.2.16) has been obtained with a simple integration by parts, and the expression on the right side of the second equality has been obtained with the help of (3.2.12). The introduction of a catastrophic risk into the model acts as if the model's rate of time preference, the discount factor, was a time varying rate, but in this case, depending on stochastic process described by a Poisson process.

The problem faced by the central planner is to find a control policy $t \rightarrow\left(K_{0}(t), K_{1}(t)\right.$, $x(t), c(t)), t \geq 0$, to maximize (3.2.16) subject to

$$
\begin{gathered}
K_{0}(t)+K_{1}(t)=K(t), \\
A K_{1}^{\alpha}(t)\left(x(t)+\beta K_{0}(t)\right)^{1-\alpha}=c(t)+k(t),
\end{gathered}
$$




$$
\begin{aligned}
\frac{d X}{d t} & =-x(t), \\
X(0) & =X_{0}>0 \text { is given, } \\
\frac{d K}{d t} & =k(t), \\
K(0) & =K_{0}>0 \text { is given, }
\end{aligned}
$$

and

$$
\begin{aligned}
& \frac{d H}{d t}=x(t)-\delta H(t), \\
& H(0)=H_{0} \text { is given. }
\end{aligned}
$$

Taking into consideration the budget constraints (3.2.18) and (3.2.17), the central planner's problem can be restated more compactly as:

$$
\max _{\left(K_{1}(t), x(t), c(t)\right)_{t \geq 0}} \int_{0}^{\infty}\left(e^{-\rho t} u(c(s)) e^{-\lambda \int_{0}^{t} H(s) d s}\right) d t
$$

subject to

$$
\begin{gathered}
\frac{d X}{d t}=-x(t), \\
X(0)=X_{0}>0 \text { is given, } \\
\frac{d K}{d t}=A K_{1}^{\alpha}(t)\left(x(t)+\beta\left(K(t)-K_{1}(t)\right)\right)^{1-\alpha}-c(t), \\
K(0)=K_{0}>0 \text { is given, }
\end{gathered}
$$

and

$$
\begin{aligned}
\frac{d H}{d t} & =x(t)-\delta H(t) \\
H(0) & =H_{0} \text { is given. }
\end{aligned}
$$

A control policy $t \rightarrow\left(K_{1}(t), x(t), c(t)\right), t \geq 0$, is said to be admissible if it is non-negative and piecewise right continuous. An admissible control is feasible if the solution of the system of differential equations that it induces is such that $X(t) \geq 0$, $K(t) \geq 0$, and $K_{1}(t) \leq K(t)$ for all $t \geq 0$. 


\subsection{The Hamilton-Jacobi-Bellman Equation}

Let $v(\xi, \eta, \zeta, \tau)$ denote the optimal expected discounted welfare, given that the economy begins at time $\tau$ with $\xi$ as the remaining stock of oil, $\eta$ as the stock of capital, and $\zeta$ as the stock of greenhouse gases in the atmosphere. More precisely,

$$
v(\xi, \eta, \zeta, \tau)=\max _{\left(K_{1}(t), x(t), c(t)\right)_{t \geq \tau}} \int_{\tau}^{\infty}\left(e^{-\rho t} u(c(s)) e^{-\lambda \int_{\tau}^{t} H(s) d s}\right) d t
$$

subject to

$$
\begin{gathered}
\frac{d X}{d t}=-x(t), \\
X(\tau)=\xi, \\
\frac{d K}{d l}=A K_{1}^{\alpha}(t)\left(x(t)+\beta\left(K(t)-K_{1}(t)\right)\right)^{1-\alpha}-c(t), \\
K(\tau)=\eta,
\end{gathered}
$$

and

$$
\begin{aligned}
\frac{d H}{d t} & =x(t)-\delta H(t), \\
H(\tau) & =\zeta
\end{aligned}
$$

In Appendix A, I show that $v(\xi, \eta, \zeta, \tau)$ satisfies the following Hamilton-Jacobi-Bellman equation:

$$
\begin{gathered}
0=\max _{\left(K_{1}, x, c\right)}\left\{e^{-\rho \tau} u(c)-D_{1} v(\xi, \eta, \zeta, \tau) x+D_{2} v(\xi, \eta, \zeta, \tau)\left(A K_{1}^{\alpha}\left(x+\beta\left(\eta-K_{1}\right)\right)^{1-\alpha}-c\right)\right. \\
\left.\quad+D_{3} v(\xi, \eta, \zeta, \tau)(x-\delta \zeta)-\lambda \zeta v(\xi, \eta, \zeta, \tau)+D_{4} v(\xi, \eta, \zeta, \tau)\right\},
\end{gathered}
$$

and the optimal capital allocated to the consumption good sector, the optimal oil extraction rate, and the optimal consumption when the economy is in state $(\xi, \eta, \zeta, \tau)$ are, respectively, the values of $K_{1}, x$, and $c$ that solve the maximization problem in $(3.3 .5)$ 


\subsection{Maximization of the Hamiltonian}

Let $\left(K_{1}^{*}(t), x^{*}(t), c^{*}(t)\right), t \geq 0$, be the solution of the central planner's problem as stated by (3.2.22) (3.2.25). Under the optimal control policy, the remaining stock of oil evolves according to the following differential equation:

$$
\begin{aligned}
& \frac{d X}{d t}=-x(t), \\
& X(0)>0 \text { is given, }
\end{aligned}
$$

and the stock of capital evolves according to the following differential equation

$$
\begin{gathered}
\frac{d K^{*}}{d t}=A\left(K_{1}^{*}(t)\right)^{\alpha}\left(x^{*}(t)+\beta\left(K^{*}(t)-K_{1}^{*}(t)\right)\right)^{1-\alpha}-c^{*}(t), \\
K^{*}(0)=K_{0}>0 \text { is given, }
\end{gathered}
$$

As for the stock of greenhouse gases in the atmosphere, its motion is governed by the following differential equation:

$$
\begin{gathered}
\frac{d H^{*}}{d t}=x^{*}(t)-\delta H^{*}(t), \\
H^{*}(0)=H_{0} \geq \underline{H} \text { is given }
\end{gathered}
$$

Next, let

$$
\begin{aligned}
& \phi(t)=D_{1} v\left(X^{*}(t), K^{*}(t), H^{*}(t), t\right), \\
& \varphi(t)=D_{2} v\left(X^{*}(t), K^{*}(t), H^{*}(t), t\right),
\end{aligned}
$$

and

$$
\psi(t)=D_{3} v\left(X^{*}(t), K^{*}(t), H^{*}(t), t\right)
$$

denote, respectively, the shadow price of oil, the shadow price of capital, and the shadow price of greenhouse gases, all at time $t$ along the optimal path. 


\subsubsection{The motion of the shadow prices}

In Appendix A, I derive the following differential equations, which govern the motion of the shadow price of oil, the shadow price of capital, and the shadow price of greenhouse gases, respectively,

$$
\begin{gathered}
\frac{d \phi}{d t}=-D_{1} \mathscr{H}\left(X^{*}(t), K^{*}(t), H^{*}(t), K_{1}^{*}(t), x^{*}(t), c^{*}(t), \phi(t), \varphi(t), \psi(t), t\right) \\
=\lambda H^{*}(t) \phi(t), \\
\frac{d \varphi}{d t}=-D_{2} \mathscr{H}\left(X^{*}(t), K^{*}(t), H^{*}(t), K_{1}^{*}(t), x^{*}(t), c^{*}(t), \phi(t), \varphi(t), \psi(t), t\right) \\
=-\varphi(t)\left(A\left(K_{1}^{*}(t)\right)^{\alpha}\left(x^{*}(t)+\beta\left(K^{*}(t)-K_{1}^{*}(t)\right)^{-\alpha} \beta(1-\alpha)-\lambda H^{*}(t)\right) .\right.
\end{gathered}
$$

and

$$
\begin{aligned}
\frac{d \psi}{d l} & =-D_{3} \mathcal{H}\left(X^{*}(t), K^{*}(t), H^{*}(t), K_{1}^{*}(t), x^{*}(t), c^{*}(t), \phi(t), \varphi(t), \psi(t), t\right) \\
& =\left(\delta+\lambda H^{*}(t)\right) \psi(t)+\lambda v\left(X^{*}(t), K^{*}(t), H^{*}(t), t\right),
\end{aligned}
$$

where the Hamiltonian is defined by

$$
\begin{aligned}
\mathscr{H}\left(X, K, H, K_{1}, x, c, \phi, \varphi, \psi, t\right)=e^{-\rho t} u(c) & -\phi x+\varphi\left(A K_{1}^{\alpha}\left(x+\beta\left(K-K_{1}\right)\right)^{1-\alpha}-c\right) \\
& +\psi(x-\delta H)-\lambda H v(X, K, H, t) .
\end{aligned}
$$

Observe that on the right side of $(3.4 .10)$ the first line has the familiar appearance of the Hamiltonian of the standard Ramsey problem, which is the instantaneous utility, minus the cost of oil depletion, plus the value of investment, minus the cost of atmospheric pollution $(\psi<0)$. In the present model, with the possible occurrence of a catastrophe, there is an additional term - on the second line of (3.4.10)- which captures the possible welfare loss when a catastrophe occurs.

The solution of (3.4.7) is

$$
\phi(t)=\phi(0) e^{\lambda \int_{0}^{t} H^{*}(s) d s}
$$


Let

$$
\begin{aligned}
\hat{\phi}(t) & =e^{\rho t} \phi(t) \\
& =\hat{\phi}(0) e^{\rho t+\lambda \int_{0}^{t} H^{*}(s) d s}
\end{aligned}
$$

denote the current shadow price of oil. In the standard model of resource depletion, the current shadow price of the exhaustible resources rises exponentially at the rate of discount, a result known as Hotelling rule. In my model, the shadow price of oil rises exponentially at a rate above the rate of discount as can be seen from (3.4.12). More precisely, the rate at which the shadow price of oil rises through time now contains a risk premium, $\lambda \int_{0}^{t} H(s) d s$, on top of the rate of discount $\rho$. The risk premium captures the threat of a catastrophe induced by climate change. Equation (3.4.12) thus extends Hotelling rule into the world of catastrophic climate change.

The solution of $(3.4 .8)$ is

$$
\varphi(t)=\varphi(0)\left(e^{-\int_{0}^{t} A\left(K_{1}^{*}(s)\right)^{\alpha}\left(x^{*}(s)+\beta\left(K^{*}(s)-K_{1}^{*}(s)\right)\right)^{-\alpha} \beta(1-\alpha) d s}\right) e^{\lambda \int_{0}^{t} H^{*}(s) d s}
$$

Let

$$
\begin{aligned}
\hat{\varphi}(t) & =e^{\rho t} \varphi(t) \\
& =\hat{\varphi}(0)\left(e^{-\int_{0}^{t} A\left(K_{1}^{*}(s)\right)^{\alpha}\left(x^{*}(s)+\beta\left(K^{*}(s)-K_{1}^{*}(s)\right)\right)^{-\alpha} \beta(1-\alpha) d s}\right) e^{\rho t+\lambda \int_{0}^{t} H^{*}(s) d s}
\end{aligned}
$$

denote the current shadow price of capital. Unlike the current shadow price of oil, it is not clear whether current shadow price of capital rises or falls through time because the first exponential term on the right-hand side of (3.4.14) declines as $t$ increases, while the second exponential term, capturing the risk of climate change, increases with time.

The solution of $(3.4 .9)$ is

$$
\psi(t)=\psi(0)\left(e^{\delta t+\lambda \int_{0}^{t} H^{*}(s) d s}\right)\left(e^{\lambda \int_{0}^{t} v\left(X^{*}(s), K^{*}(s), H^{*}(s), s\right) d s}\right) .
$$


Let

$$
\begin{aligned}
\hat{\psi}(t) & =e^{\rho t} \psi(t) \\
& =\hat{\psi}(0)\left(e^{\delta t+\lambda \int_{0}^{t} v\left(X^{*}(s), K^{* *}(s), H^{*}(s), s\right) d s}\right) e^{\rho t+\lambda \int_{0}^{t} H^{*}(s) d s}
\end{aligned}
$$

denote the current shadow price of greenhouse gases. Note that the current shadow price of greenhouse gases rises through time at a rate which is even higher than the rate at which the current shadow price of oil rises in time: in addition to the risk premium associated with the threat of catastrophic climate change, the rate at which the current shadow price of greenhouse gases now contains an exponential term, namely $e^{\delta t+\lambda \int_{0}^{t} r\left(X^{*}(s) \cdot K^{*}(s), H^{*}(s), s\right) d s}$, whose rate of change also rises through time.

\subsubsection{The first-order conditions}

Applying the Hamilton-Jacobi-Bellman equation (3.3.5) for $(\xi, \eta, \zeta, \tau)=\left(X^{*}, K^{*}, H^{*}, t\right)$, and then slightly rearranging the result, and from now and on I drop the time argument from the state, costate and control functions unless it is ambiguous, we obtain

$$
\begin{array}{r}
D_{4} v\left(X^{*}, K^{*}, H^{*}, t\right)=\max _{\left(K_{1}, x, c\right)}\left\{e^{-\rho t} \frac{c^{1-\theta}}{1-\theta}-\phi x+\varphi\left(A K_{1}^{\alpha}\left(x+\beta\left(K^{*}-K_{1}\right)\right)^{1-\alpha}-c\right)\right. \\
\left.+\psi\left(x-\delta H^{*}\right)-\lambda H^{*} v\left(X^{*}, K^{*}, H^{*}, t\right)\right\}
\end{array}
$$

Now because the differential equations that govern the motion of the state of the system are autonomous, and because time enters the objective function as a discounting factor, we have $v(X . K, H, t)=e^{-\rho t} v(X, K, H, 0)$, from which we obtain $D_{4} v(X, K, H, t)=-\rho e^{-\rho t} v(X, K, H, 0)$. Using this last result in (3.4.16), we obtain

$$
\begin{array}{r}
\rho v\left(X^{*}, K^{*}, H^{*}, 0\right)=\max _{\left(K_{1}, x, c\right)}\left\{\frac{c^{1-\theta}}{1-\theta}-\hat{\phi} x+\hat{\varphi}\left(A K_{1}^{\alpha}\left(x+\beta\left(K^{*}-K_{1}\right)^{1-\alpha}-c\right)\right)\right. \\
\left.+\hat{\psi}\left(x-\delta H^{*}\right)-\lambda H^{*} v\left(X^{*}, K^{*}, H^{*}, 0\right)\right\}
\end{array}
$$


The optimal consumption at time $t$ is value of $c$ that maximizes the expression on the right-hand side of (3.4.18) is given by

$$
\begin{aligned}
c^{*} & =(\hat{\varphi})^{-\frac{1}{\theta}} \\
& =\left(\hat{\varphi}(0)\left(e^{-\int_{0}^{t} A\left(K_{1}^{*}(s)\right)^{\alpha}\left(x^{*}(s)+\beta\left(K^{*}(s)-K_{1}^{*}(s)\right)\right)^{-\alpha} \beta(1-\alpha) d s}\right) e^{\rho t+\lambda \int_{0}^{t} H^{*}(s) d s}\right)^{-\frac{1}{\theta}} .
\end{aligned}
$$

The following first-order condition characterizes the optimal allocation of capital to the consumption good sector:

$$
\begin{aligned}
& A \alpha\left(K_{1}^{*}\right)^{\alpha-1}\left(x^{*}+\beta\left(K^{*}-K_{1}^{*}\right)\right)^{1-\alpha}-\left(K_{1}^{*}\right)^{\alpha}\left(x^{*}+\beta\left(K^{*}-K_{1}^{*}\right)\right)^{-\alpha} \beta(1-\alpha)= \\
& A\left(K_{1}^{*}\right)^{\alpha}\left(x^{*}+\beta\left(K^{*}-K_{1}^{*}\right)\right)^{-\alpha}\left(\alpha\left(K_{1}^{*}\right)^{-1}\left(x^{*}+\beta\left(K^{*}-K_{1}^{*}\right)\right)-\beta(1-\alpha)\right) \geq 0,
\end{aligned}
$$

with equality holding if $K_{1}^{*}<K^{*}$. This first-order condition asserts that given the oil extraction rate $x^{*}$, capital is allocated between the consumption good sector and the renewable energy sector to maximize output.

Note that the first-order condition $(3.4 .20)$ can be reduced to

$$
\alpha\left(K_{1}^{*}\right)^{-1}\left(x^{*}+\beta\left(K^{*}-K_{1}^{*}\right)\right)-\beta(1-\alpha) \geq 0
$$

with equality holding if $K_{1}^{*}<K^{*}$.

The following first-order condition characterizes the optimal oil extraction rate.

$$
-\hat{\phi}+\hat{\psi}+\hat{\varphi} A\left(K_{1}^{*}\right)^{\alpha}\left(x^{*}+\beta\left(K^{*}-K_{1}^{*}\right)\right)^{-\alpha}(1-\alpha) \leq 0
$$

with equality holding if $x^{*}>0$.

The first-order condition (3.4.22) asserts that the marginal product of oil converted into welfare through the shadow price of capital $\hat{\varphi}$ is equal to the shadow price of oil minus the shadow price of greenhouse gases whenever fossil fuels are burned to provide part of the energy input used for the production of the consumption good. 
Thus, at the margin, the contribution of the fossil fuels resides in the extra output they help to produce, while the cost consists of the depletion cost $(\hat{\phi}>0)$ and the cost of atmospheric pollution $(\hat{\psi}<0)$.

\subsection{Green national income}

At any point on the optimal trajectory, (3.4.18) takes on the following form:

$$
\begin{aligned}
\rho v\left(X^{*}, K^{*}, H^{*}, 0\right)=u\left(c^{*}\right)-\hat{\phi} \cdot x^{*} & +\hat{\varphi} \cdot A\left(K_{1}^{*}\right)^{\alpha}\left(\left(x^{*}+\beta\left(K^{*}-K_{1}^{*}\right)\right)^{1-\alpha}-c^{*}\right) \\
& +\hat{\psi} \cdot\left(x^{*}-\delta H^{*}\right)-\lambda H^{*} v\left(X^{*}, K^{*}, H^{*}, 0\right) .
\end{aligned}
$$

Observe that in (3.5.1), the expression on the left-hand side represents national income at time $t$, and the expression on the right-hand side is the current-value Hamiltonian at time $t$ along the optimal trajectory. Equation (3.5.1) asserts that at each instant, national income is equal to the current Hamiltonian. On the right-hand side of (3.5.1), the first term, $u\left(c^{*}\right)$, is the social welfare obtained from consumption at time $t$, and this is counted as part of national income. The second term, $\hat{\phi} \cdot x^{*}$, represents the cost of oil depletion at time $t$. Because depletion reduces the stock of fossil fuels, this expression enters negatively in the calculation of national income as depletion cost. The third term, $\hat{\varphi} \cdot A\left(K_{1}^{*}\right)^{\alpha}\left(\left(x^{*}+\beta\left(K^{*}-K_{1}^{*}\right)\right)^{1-\alpha}-c^{*}\right)$, represents the value of the investment made at time $t$. The fourth tern, $\hat{\psi} \cdot\left(x^{*}-\delta H^{*}\right)$, represents the cost induced by the rise in the stock of greenhouse gases in the atmosphere. Because the shadow price of greenhouse gases is negative, this term captures the degradation of the environment caused by the burning of fossil fuels. Finally, the last term, $\lambda H^{*} v\left(X^{*}, K^{*}, H^{*}, 0\right)$, represents the expected loss in discounted welfare discounted to time $t$ due to the possibility of a catastrophe. Equation (3.5.1) thus provides a method for computing green national income in which the damages to the environment and the threat to climate change are accounted for. 


\subsection{A characterization of the optimal solution}

Lemma 1. At any time, only one source of energy fossil fuels or renewable energy, but not both is exploited to provide the energy used in the production of the consumption good.

Proof. Lemma 1 is proved by reductio ad absurdum. Suppose that for some time $t$, both $x^{*}>0$ and $K_{1}^{*}<K^{*}$. That is, the solution of the maximization of the Hamiltonian is an interior solution. Under such a scenario, (3.4.21) and (3.4.22) both hold with equality:

$$
\begin{gathered}
\alpha\left(K_{1}^{*}(t)\right)^{-1}\left(x^{*}(t)+\beta\left(K^{*}(t)-K_{1}^{*}(t)\right)\right)-\beta(1-\alpha)=0, \\
-\hat{\phi}(l)+\hat{\psi}(l)+\hat{\varphi}(l) \Lambda\left(K_{1}^{*}(l)\right)^{\alpha}\left(x^{*}(l)+\beta\left(K^{*}(t)-K_{1}^{*}(t)\right)\right)^{-\alpha}(1-\alpha)=0,
\end{gathered}
$$

Solving (3.6.1) for $K_{1}^{*}$, gives

$$
K_{1}^{*}(t)=\frac{\alpha\left(x^{*}(t)+\beta K^{*}(t)\right)}{\beta} .
$$

Using (3.6.3) in (3.6.2), we obtain after some simplifications

$$
-\hat{\phi}(t)+A(1-\alpha)^{1-\alpha} \alpha^{\alpha} \beta^{-\alpha} \hat{\varphi}(t)+\hat{\psi}(t)=0
$$

Because the controls are piecewise and right-continuous, (3.6.4) must hold in a right neighborhood of $t$. I now show that this is not possible. To this end, consider a time $\tau$ in a right neighborhood of $t$, and then divide (3.6.4) by $\hat{\phi}(\tau)$ to obtain

$$
-1+A(1-\alpha)^{1-\alpha} \alpha^{\alpha} \beta^{-\alpha} \frac{\hat{\varphi}(\tau)}{\hat{\phi}(\tau)}+\frac{\hat{\psi}(\tau)}{\hat{\phi}(\tau)}=0 .
$$


Using the expressions for $\hat{\phi}(\tau), \hat{\varphi}(\tau)$ : and $\hat{\psi}(\tau)$, we can rewrite (3.6.6) as

$$
\begin{array}{r}
-1+A(1-\alpha)^{1-\alpha}\left(\frac{\alpha}{\beta}\right)^{\alpha} \times \\
\frac{\left(\hat{\varphi}(0)\left(e^{-\int_{0}^{\tau} A\left(K_{\mathrm{i}}^{*}(s)\right)^{\alpha}\left(x^{*}(s)+\beta\left(K^{*}(s)-K_{1}^{*}(s)\right)\right)^{-\alpha} \beta(1-\alpha) d s}\right) e^{\rho \tau+\lambda \int_{0}^{\tau} H^{*}(s) d s}\right)}{\left(\hat{\phi}(0) e^{\rho \tau+\lambda \int_{0}^{\tau} H^{*}(s) d s}\right)} \\
+\frac{\hat{\psi}(0)\left(e^{\delta \tau+\lambda \int_{0}^{\tau} v\left(X^{*}(s) \cdot K^{*}(s), H^{*}(s), s\right) d s}\right) e^{\rho \tau+\lambda \int_{0}^{\tau} H^{*}(s) d s}}{\hat{\phi}(0) e^{\rho \tau+\lambda \int_{0}^{\tau} H^{*}(s) d s}}=0
\end{array}
$$

which can be simplified to

$$
\begin{gathered}
-1+A(1-\alpha)^{1-\alpha}\left(\frac{\alpha}{\beta}\right)^{\alpha} \frac{\hat{\varphi}(0)}{\hat{\phi}(0)}\left(e^{-\int_{0}^{\tau} A\left(K_{1}^{*}(s)\right)^{\alpha}\left(x^{*}(s)+\beta\left(K^{*}(s)-K_{1}^{*}(s)\right)\right)^{-\alpha} \beta(1-\alpha) d s}\right) \\
+\frac{\hat{\psi}(0)}{\hat{\phi}(0)}\left(e^{\delta \tau+\lambda \int_{0}^{\tau} v\left(X^{*}(s), K^{*}(s), H^{*}(s), s\right) d s}\right)=0 .
\end{gathered}
$$

On the left-hand side of $(3.6 .7)$, the first term is -1 , which is a constant. The second term is

$$
A(1-\alpha)^{1-\alpha}\left(\frac{\alpha}{\beta}\right)^{\alpha} \frac{\hat{\varphi}(0)}{\hat{\phi}(0)}\left(e^{-\int_{0}^{T} A\left(K_{1}^{*}(s)\right)^{\alpha}\left(x^{*}(s)+\beta\left(K^{*}(s)-K_{1}^{*}(s)\right)\right)^{-\alpha} \beta(1-\alpha) d s}\right)>0,
$$

and strictly decreasing in $\tau$. The third term is

$$
\frac{\hat{\psi}(0)\left(e^{\delta \tau+\lambda \int_{0}^{\tau} v\left(X^{*}(s), K^{*}(s), H^{*}(s), s\right) d s}\right)}{\hat{\phi}(0)}<0,
$$

because $\hat{\psi}(0)<0, \hat{\phi}(0)>0$, and is strictly decreasing in $\tau$. Thus, the left-hand side of (3.6.7) is strictly decreasing in $\tau$, and thus (3.6.7) cannot hold in a right neighborhood of $t$.

According to Lemma 1, only one source of energy is exploited at any instant. Thus, when the backstop is exploited, we must have $x^{*}=0$, and it follows from (3.6.3) that

$$
\left.K_{1}^{*}(t)=\alpha K^{*}(t)\right)
$$


On the other hand, if the fossil fuel technology is exploited, i.e., if $x^{*}>0$, then $K_{1}^{*}=K^{*}$, and solving (3.6.2) for $x^{*}$, we obtain

$$
x^{*}(t)=A^{\frac{1}{\alpha}}(1-\alpha)^{\frac{1}{\alpha}}\left(\frac{\hat{\varphi}(t)}{\hat{\phi}(t)-\hat{\psi}(t)}\right)^{\frac{1}{\alpha}} K^{*}(t) .
$$

Lemma 2. The stock of fossil fuels will be exhausted in finite time.

Proof. Lemma 2 is proved by reductio ad absurdum. Suppose that the stock of fossil fuels is not depleted in finite time. Then for any time $s$ arbitrarily large, there is a time $t>s$, such that $x^{*}>0$. Under such a scenario, the output of the consumption good at time $t$ is given by

$$
\begin{aligned}
A\left(K^{*}(t)\right)^{\alpha}\left(x^{*}(t)\right)^{1-\alpha} & =A\left(K^{*}(t)\right)^{\alpha}\left(A^{\frac{1}{\alpha}}(1-\alpha)^{\frac{1}{\alpha}}\left(\frac{\hat{\varphi}(t)}{\hat{\phi}(t)-\hat{\psi}(t)}\right)^{\frac{1}{\alpha}} K^{*}(t)\right)^{1-\alpha} \\
& =A K^{*}(t)(1-\alpha)^{\frac{1-\alpha}{\alpha}}\left(\frac{\hat{\varphi}(t)}{\hat{\phi}(t)-\hat{\psi}(t)}\right)^{\frac{1-\alpha}{\alpha}}
\end{aligned}
$$

On the other hand, if the central planner chooses not to exploit the fossil fuel technology at time $l$, then the output of the consumption good at this instant is given by

$$
A\left(\alpha K^{*}(t)\right)^{\alpha}\left(\beta(1-\alpha) K^{*}(t)\right)^{1-\alpha}=A \alpha^{\alpha} K^{*}(t)(\beta(1-\alpha))^{1-\alpha}
$$

Dividing (3.6.10) by (3.6.11), we obtain

$$
\begin{aligned}
\frac{A\left(K^{*}(t)\right)^{\alpha}\left(x^{*}(t)\right)^{1-\alpha}}{A\left(\alpha K^{*}(t)\right)^{\alpha}\left(\beta(1-\alpha) K^{*}(t)\right)^{1-\alpha}} & =\frac{A K^{*}(t)\left((1-\alpha) \frac{\hat{\varphi}(t)}{\hat{\phi}(t)-\hat{\psi}(t)}\right)^{\frac{1-\alpha}{\alpha}}}{A \alpha^{\alpha} K^{*}(t)(\beta(1-\alpha))^{1-\alpha}} \\
& =\frac{(1-\alpha)^{\frac{(1-\alpha)^{2}}{\alpha}}}{\alpha^{\alpha} \beta^{1-\alpha}}\left(\frac{\hat{\varphi}(t)}{\hat{\phi}(t)-\hat{\psi}(t)}\right)^{\frac{1-\alpha}{\alpha}} .
\end{aligned}
$$


Now using (3.4.12), (3.4.14), and (3.4.16), we obtain

$$
\begin{aligned}
\frac{\hat{\varphi}(t)}{\hat{\phi}(t)-\hat{\psi}(t)} & =\frac{\hat{\varphi}(0)\left(e^{-\int_{0}^{t} A\left(K_{1}^{*}(s)\right)^{\alpha}\left(x^{*}(s)+\beta\left(K^{*}(s)-K_{1}^{*}(s)\right)\right)^{-\alpha} \beta(1-\alpha) d s}\right) e^{\rho t+\lambda \int_{0}^{t} H^{*}(s) d s}}{\left(\hat{\phi}(0)-\hat{\psi}(0)\left(e^{\delta t+\lambda \int_{0}^{t} v\left(X^{*}(s) \cdot K^{*}(s), H^{*}(s), s\right) d s}\right)\right) e^{\rho t+\lambda \int_{0}^{t} H^{*}(s) d s}} \\
& =\frac{\hat{\varphi}(0)\left(e^{-\int_{0}^{t} A\left(K_{1}^{*}(s)\right)^{\alpha}\left(x^{*}(s)+\beta\left(K^{*}(s)-K_{1}^{*}(s)\right)\right)^{-\alpha}} \beta(1-\alpha) d s\right.}{\hat{\phi}(0)-\hat{\psi}(0)\left(e^{\delta t+\lambda \int_{0}^{t} v\left(X^{*}(s), K^{*}(s), H^{*}(s), s\right) d s}\right)},
\end{aligned}
$$

which is strictly decreasing as $t$ increases. Furthermore, it tends to 0 when $t \rightarrow \infty$. Hence the ratio represented by (3.6.12) tends to 0 when $t \rightarrow \infty$, which means that it is not optimal to exploit the fossil fuel technology when $t$ is large, contradicting the premise of the reductio ad absurdum argument.

Lemma 3. The backstop will not be exploited until the stock of fossil fuels has been depleted.

Proof. Lemma 3 is proved by reductio ad absurdum. Suppose that the backstop is exploited at some time $l$ when the stock of fossil fuels has not been exhausted, i.e., $K_{1}^{*}<K^{*}$ and $X^{*}>0$. According to Lemma 1, the fossil fuel technology is not exploited at time $t$, i.e., we have $x^{*}=0$, and, using (3.6.8), we obtain $K_{1}^{*}=\alpha K^{*}$. Furthermore, because the controls are piecewise and right continuous, we must have

$$
\begin{aligned}
K_{1}^{*}(s) & =\alpha K^{*}(s), \\
x^{*}(s) & =0, \quad t \leq s<t+\epsilon,
\end{aligned}
$$

where $\epsilon$ is a small positive number.

Because $X^{*}>0$, and because under the optimal solution the stock of fossil fuels must be depleted, the extraction rate must be positive at some time after $t+\epsilon$. Thus, if we let

$$
\tau=\inf \left\{s \geq t \mid x^{*}(s)>0\right\}
$$


denote the first time after $t$ the extraction rate is positive, then according to (3.6.9), we must have

$$
\begin{gathered}
K_{1}^{*}(\tau)=K^{*}(\tau), \\
x^{*}(\tau)=A^{\frac{1}{\alpha}}(1-\alpha)^{\frac{1}{\alpha}}\left(\frac{\hat{\varphi}(\tau)}{\hat{\phi}(\tau)-\hat{\psi}(\tau)}\right)^{\frac{1}{\alpha}} K^{*}(\tau)>0 .
\end{gathered}
$$

That is, the fossil fuel technology is exploited in a right neighborhood of $\tau$. It also follows from the definition of $\tau$ that the backstop is exploited during the time interval $[t, \tau)$. By continuity, at the interface between the two regimes, i.e., at time $\tau$, either one of the two technologies can be exploited under the optimal solution. More precisely, at time $\tau$, the policy represented by (3.6.17) as well as the policy that is the limit of (3.6.15) when $s \uparrow \tau$, namely

$$
\begin{aligned}
& \lim _{s \uparrow \tau} K_{1}^{*}(s)=\alpha K^{*}(\tau), \\
& \lim _{s \uparrow \tau} x^{*}(s)=0 .
\end{aligned}
$$

both maximize the Hamiltonian at time $\tau$, and this means

$$
\begin{aligned}
\mathscr{H}\left(X^{*}(\tau), K^{*}(\tau),\right. & \left.H^{*}(\tau), \alpha K^{*}(\tau), 0, c^{*}(\tau), \hat{\phi}(\tau), \hat{\varphi}(\tau), \hat{\psi}(\tau), \tau\right)= \\
& \mathcal{H}\left(X^{*}(\tau), K^{*}(\tau), H^{*}(\tau), K^{*}(\tau), x^{*}(\tau), c^{*}(\tau), \hat{\phi}(\tau), \hat{\varphi}(\tau), \hat{\psi}(\tau), \tau\right),
\end{aligned}
$$


i.e.,

$$
\begin{aligned}
& e^{-\rho \tau} \frac{\left(c^{*}(\tau)\right)^{1-\theta}}{1-\theta}+\hat{\varphi}(\tau)\left(A \alpha\left(K^{*}(\tau)\right)^{\alpha} \beta(1-\alpha)\left(K^{*}(\tau)\right)^{1-\alpha}-c^{*}(\tau)\right) \\
& -\hat{\psi}(\tau) \delta H^{*}(\tau)-\lambda H^{*}(\tau) v\left(X^{*}(\tau), K^{*}(\tau), H^{*}(\tau), \tau\right)= \\
& e^{-\rho \tau} \frac{\left(c^{*}(\tau)\right)^{1-\theta}}{1-\theta}-\hat{\phi}(\tau) A^{\frac{1}{\alpha}}(1-\alpha)^{\frac{1}{\alpha}}\left(\frac{\hat{\varphi}(\tau)}{\hat{\phi}(\tau)-\hat{\psi}(\tau)}\right)^{\frac{1}{\alpha}} K^{*}(\tau) \\
& +\hat{\varphi}(\tau)\left(A\left(K^{*}(\tau)\right)^{\alpha}\left(A^{\frac{1}{\alpha}}(1-\alpha)^{\frac{1}{\alpha}}\left(\frac{\hat{\varphi}(\tau)}{\hat{\phi}(\tau)-\hat{\psi}(\tau)}\right)^{\frac{1}{\alpha}}\left(K^{*}(\tau)\right)^{1-\alpha}-c^{*}(\tau)\right)\right) \\
& +\hat{\psi}(\tau)\left(A^{\frac{1}{\alpha}}(1-\alpha)^{\frac{1}{\alpha}}\left(\frac{\hat{\varphi}(\tau)}{\hat{\phi}(\tau)-\hat{\psi}(\tau)}\right)^{\frac{1}{\alpha}} K^{*}(\tau)-\delta H^{*}(\tau)\right) \\
& -\lambda H^{*}(\tau) v\left(X^{*}(\tau), K^{*}(\tau), H^{*}(\tau), \tau\right) .
\end{aligned}
$$

which after simplification, (3.6.19) reduces to

$$
\frac{A \alpha^{\alpha} \beta^{1-\alpha}(1-\alpha)^{1-\alpha}}{A^{\frac{1}{\alpha}}(1-\alpha)^{\frac{1}{\alpha}}}=\frac{\alpha}{1-\alpha}\left(\frac{\hat{\varphi}(\tau)}{\hat{\phi}(\tau)-\hat{\psi}(\tau)}\right)^{\frac{1-\alpha}{\alpha}}
$$

After time $\tau$, there are two possibilities: (i) $x^{*}(s)>0$ for all $s \geq \tau$, and (ii) $x^{*}(s)=0$ for some $s>\tau$. Possibility (i) is ruled out by Lemma 2. Under possibility (ii), let $\tau^{\prime}$ be the first time after $\tau$ that the backstop is exploited again. At the interface $\hat{\tau}$, the same argument used to obtain (3.6.20) can be deployed to obtain the following version of $(3.6 .20)$

$$
\frac{\Lambda \alpha^{\alpha} \beta^{1-\alpha}(1-\alpha)^{1-\alpha}}{A^{\frac{1}{\alpha}}(1-\alpha)^{\frac{1}{\alpha}}}=\frac{\alpha}{1-\alpha}\left(\frac{\hat{\varphi}\left(\tau^{\prime}\right)}{\hat{\phi}\left(\tau^{\prime}\right)-\hat{\psi}\left(\tau^{\prime}\right)}\right)^{\frac{1-\alpha}{\alpha}} .
$$

However, we have argued that the expression represented by (3.6.13) is strictly decreasing in $t$. Hence (3.6.20) and (3.6.21) are mutually incoherent, The premise of the reductio ad absurdum argument leads to a contradiction.

Proposition 1. Under the social optimum, the fossil fuel technology is exploited first, and the backstop is only brought into use after the stock of fossil fuels has been depleted. Furthermore, the technology substitution occurs in finite time. 
Proof. The first statement of Proposition 1 follows immediately from Lemma 3, which asserts that the backstop is not exploited as long as the remaining stock of fossil fuels is still positive. The second statement of Proposition 1 follows from Lemma 2, which asserts that the stock of fossil fuels is exhausted in finite time.

According to Proposition 1, the fossil fucls are exploited first and exhausted in finite time. Only after the stock of fossil fuels has been depleted is the backstop brought into use. The technology substitution occurs abruptly, and there are only two phases in the evolution of the economy: the fossil fuel phase and the post fossil fuel phase. A transitional phase during which the two energy technologies co-exist does not exist. The economic intuition behind the abrupt technology substitution is that there is perfect capital mobility between the consumption good sector and the renewable-energy sector. If we adopt the assumption that capital is sector-specific, then a transitional phase must exist to allow for the accumulation of backstop capital so that a positive level of backstop capital must exists at the time the resource is exhausted.

Let $T$ denote the time at which the stock of fossil fuels is exhausted. At any instant after time $T$, the stock of greenhouse gases in the atmosphere is given by

$$
H^{*}(t)=H^{*}(T) e^{-\delta(t-T)}, \quad t \geq T .
$$

Furthermore, after resource exhaustion, we have

$$
x^{*}(t)=0, K_{1}^{*}(t)=\alpha K^{*}(t), \quad t \geq T .
$$

Using (3.6.22) and (3.6.23) in (3.4.14), we obtain the following expression for the 
shadow price of capital after resource exhaustion:

$$
\begin{aligned}
\hat{\varphi}(t) & =\hat{\varphi}(0)\left(e^{-\int_{0}^{t} A\left(K_{i}^{*}(s)\right)^{\alpha}\left(x^{*}(s)+\beta\left(K^{*}(s)-K_{i}^{*}(s)\right)\right)^{-a} \beta(1-\alpha) d s}\right) e^{\rho t+\lambda \int_{0}^{t} H^{*}(s) d s} \\
& =\varphi(0)\left(e^{-\int_{0}^{t} A \alpha^{\alpha}(\beta(1-\alpha))^{1-\alpha} d s} d s\right) e^{\rho t+\lambda \int_{0}^{t} H^{*}(T) e^{-\delta(s-T)} d s} \\
& =\varphi(0) e^{-\left(A \alpha^{\alpha} \beta^{1-\alpha}(1-\alpha)^{1-\alpha}-\rho\right) t} e^{\lambda H^{*}(T) \frac{e^{\delta} T}{\delta}\left(1-e^{-\delta t}\right)}
\end{aligned}
$$

Using (3.6.24) in (3.4.19), we obtain the following expression for the optimal consumption at an instant after resource exhaustion:

$$
\begin{aligned}
c^{*}(t) & =(\hat{\varphi}(t))^{-\frac{1}{\theta}} \\
& =\left(\varphi(0)^{-\frac{1}{\theta}} e^{-\lambda H^{*}(T) \frac{e^{\delta T}}{\delta \theta}\left(1-e^{-\delta t}\right)}\right) e^{\frac{A \alpha^{\alpha} \beta^{1-\alpha}(1-\alpha)}{\theta}} .
\end{aligned}
$$

Proposition 2. If $A \alpha^{\alpha} \beta^{1-\alpha}(1-\alpha)^{1-\alpha}-\rho>0$, then a steady state exists, in which consumption grows at a constant rate given by $\frac{A \alpha^{\alpha} \beta^{1-\alpha}(1-\alpha)^{1-\alpha}-\rho}{\theta}$. Furthermore, consumption begins its ascent before the stock of fossil fuels is exhausted.

Proof. To prove Proposition 2, first note that

$$
\lim _{t \rightarrow \infty} \varphi(0)^{-\frac{1}{\theta}} e^{-\lambda H^{*}(T) e^{\delta T} \frac{1}{\delta \theta}\left(1-e^{-\delta t}\right)}=f(0)^{-\frac{1}{\theta}} e^{-\lambda H^{*}(T) e^{\delta T} \frac{1}{\delta \theta}}
$$

Thus, when $t$ is large, the variation of the optimal consumption rate is governed by the exponential function of time $e^{\frac{A \alpha^{\alpha} \beta^{1 \alpha}(1-\alpha)^{1-\alpha}-\rho}{\theta} t}$, which grows at the constant rate $\frac{A \alpha^{\alpha} \beta^{1-\alpha}(1-\alpha)^{1-\alpha}-\rho}{\theta}$.

To prove the second statement of Proposition 2, note that the expression inside the grand parentheses on the right-hand side of (3.6.25) is strictly increasing in $t$, and this means that consumption is strictly rising after oil exhaustion. By continuity, consumption must be rising in a left neighborhood of $T$, the date of resource exhaustion.

Now even after the stock of fossil fuels has been exhausted the threat of climate change does not vanish as long as the stock of greenhouse gases in the atmosphere 
is still positive. However, once the stock of fossil fuels has been depleted, the stock of greenhouse gases will decline asymptotically to its natural level in the long run. Proposition 2 describes the evolution of the economy in the long run under the scenario that the catastrophe does not occur. As asserted by Proposition 1, in the long run the economy converges to a balanced growth path of an AK model.

Proposition 2 describes the optimal pattern of consumption after resource exhaustion. I now study the question of how the possibility of a catastrophe influences the pattern of consumption in the fossil fuel phase. First, we need to establish a preliminary result.

Lemma 4. The curve $t \rightarrow \frac{\hat{\phi}(t)-\hat{\psi}(t)}{\hat{\varphi}(t)}, t \geq 0$, is strictly increasing, and for $0 \leq t \leq T$, $\frac{\hat{\phi}(t)-\hat{\psi}(t)}{\hat{\varphi}(t)}<(1-\alpha)^{1-\alpha} \alpha^{-\frac{\alpha^{2}}{1-\alpha}} \beta^{-\alpha}$.

Proof. In the proof of Lemma 2, we have shown that $t \rightarrow \frac{\hat{\varphi}(t)}{\hat{\phi}(t)-\hat{\psi}(t)}, t \geq 0$, as given by (3.6.13), is strictly decreasing, and this means $t \rightarrow \frac{\dot{\phi}(t)-\hat{\psi}(t)}{\hat{\phi}(t)}, t \geq 0$, is strictly increasing. Now at any instant, even when the stock of fossil fuels has not been exhausted, it is always possible to exploit only the backstop. Thus, when the fossil fuel technology is exploited, the output obtained under this action must exceed the output obtained by exploiting only the backstop. Hence according to (3.6.12), the following inequality must hold before resource exhaustion:

$$
\begin{aligned}
\frac{A\left(K^{*}\right)^{\alpha}\left(x^{*}\right)^{1-\alpha}}{A\left(\alpha K^{*}\right)^{\alpha}\left(\beta(1-\alpha) K^{*}\right)^{1-\alpha}} & =\frac{A K^{*}(1-\alpha)^{\frac{1-\alpha}{\alpha}}\left(\frac{\hat{\varphi}}{\hat{\phi}-\hat{\psi}}\right)^{\frac{1-\alpha}{\alpha}}}{A \alpha^{\alpha} K^{*}(\beta(1-\alpha))^{1-\alpha}} \\
& =\frac{(1-\alpha)^{\frac{(1-\alpha)^{2}}{\alpha}}}{\alpha^{\alpha} \beta^{1-\alpha}}\left(\frac{\hat{\varphi}}{\hat{\phi}-\hat{\psi}}\right)^{\frac{1-\alpha}{\alpha}}>1, \quad 0 \leq t<T .
\end{aligned}
$$

which can be rearranged to obtain the inequality stated in Lemma 4 .

The following lemma describes the variation of the current shadow price of capital in time. It can be used to find the optimal pattern of consumption during the fossil fuel phase. 
Lemma 5. We have $\frac{\hat{\varphi}^{\prime}(t)}{\hat{\varphi}(t)}=-\beta \frac{\hat{\phi}(t)-\hat{\psi}(t)}{\hat{\varphi}(t)}+\rho+\lambda H^{*}(t), \quad 0 \leq t<T$.

Proof. Before oil exhaustion, the shadow price of capital is obtained by setting $K_{1}^{*}(s)=$ $K^{*}(s), \quad 0 \leq s<T$, in $(3.6 .13):$

$$
\hat{f}(t)=\hat{\varphi}(0)\left(e^{-\int_{0}^{t} A\left(K_{i}^{*}(s)\right)^{\alpha}\left(x^{*}(s)\right)^{-\alpha} \beta(1-\alpha) d s}\right) e^{\rho t+\lambda \int_{0}^{t} H^{*}(s) d s}, \quad 0 \leq t<T .
$$

Differentiating (3.6.26) logarithmically with respect to $t$, we obtain

$$
\begin{aligned}
\frac{\hat{\varphi}^{\prime}(t)}{\hat{\varphi}(l)} & =-A\left(K_{1}^{*}\right)^{\alpha}\left(x^{*}\right)^{-\alpha} \beta(1-\alpha)+\rho+\lambda H^{*} \\
& =-A\left(K_{1}^{*}\right)^{\alpha}\left(A^{\frac{1}{\alpha}}(1-\alpha)^{\frac{1}{\alpha}}\left(\frac{\hat{\varphi}}{\hat{\phi}-\hat{\psi}}\right)^{\frac{1}{\alpha}} K^{*}\right)^{-\alpha} \beta(1-\alpha)+\rho+\lambda H^{*} \\
& =-\beta \frac{\hat{\phi}-\hat{\psi}}{\hat{\varphi}}+\rho+\lambda H^{*}
\end{aligned}
$$

Note that the second line in (3.6.27) has been obtained by using the expression for $x^{*}(t), \quad 0 \leq t<T$, as given by (3.6.9), and the third line has been obtained by simplifying the second line.

The following proposition describes the optimal pattern of consumption during the fossil fuel phase when there is excessive atmospheric pollution at the beginning.

Proposition 3. Suppose that $A \alpha^{\alpha} \beta^{1-\alpha}(1-\alpha)^{1-\alpha}-\rho>0$. If

$$
\rho+\lambda H^{*}(0)>(1-\alpha)^{1-\alpha} \alpha^{-\frac{\alpha^{2}}{1-\alpha}} \beta^{1-\alpha}
$$

then $\hat{\varphi}^{\prime}(t)>0$ in a right neighborhood of 0 , and this means consumption is strictly decreasing at the beginning, descends to a global minimum, and then rises at some time before resource exhaustion. That is, if the initial volume of greenhouse gases in the atmosphere is large, then the optimal consumption pattern has the U-shape.

Proof. According to Lemma 4 , for $0 \leq t \leq T, \frac{\hat{\phi}(t)-\hat{\psi}(t)}{\hat{\varphi}(t)}$ is bounded above by $(1-$ $\alpha)^{1-\alpha} \alpha^{-\frac{\alpha^{2}}{1-\alpha}} \beta^{1-\alpha}$. Thus, if the condition (3.6.28) is satisfied, then the current shadow 
price of capital will be rising in a right neighborhood of 0 , and this in turn implies that consumption is falling in a right neighborhood of 0 . Furthermore, according to Proposition 2, consumption starts its climb before oil exhaustion. Thus, the optimal consumption pattern has the U-shape when the initial stock of greenhouse gases in the atmosphere is large.

The intuition behind Proposition 2 is not hard to grasp. When the initial volume of greenhouse gases in the atmosphere is high, a catastrophe is more probable, and this encourages the production of green energy. Furthermore, because the production of green energy requires backstop capital, consumption must be curtailed to accelerate the accumulation of capital. Only after a sufficient volume of capital has been accumulated is consumption allowed to rise, and this explains the U-shape of the optimal pattern of consumption through time.

Up to this point, we have denoted by $\hat{\phi}(t), \hat{\varphi}(t)$, and $\hat{\psi}(t)$, the shadow prices of oil, capital, and greenhouse gases. These shadow prices, in addition to time, also depends on $\lambda$, and to make their dependence on $\lambda$ explicit, I shall write them in what follows as $\hat{\phi}(t, \lambda), \hat{\varphi}(l, \lambda)$, and $\hat{\psi}(t, \lambda)$. Now let

$$
\omega(t, \lambda)=\frac{\hat{\phi}(t, \lambda)-\hat{\psi}(t, \lambda)}{\hat{\varphi}(t, \lambda)}
$$

which is the ratio of the shadow price of oil over the shadow price of capital at time $t=0$ for the model of resource depletion without threat of catastrophes analyzed in Essay 2 of the thesis. Then we have

Proposition 4. If $\rho+\lambda\left(X_{0}+H_{0}\right)<\beta \omega(0,0)$, then $\hat{\varphi}^{\prime}(t)<0$ for all $t \geq 0$, and this means consumption is strictly increasing through time. The hypothesis of the proposition is satisfied if $\rho$, the rate of time preferences, and $\lambda$ are both small.

Proof. First, note that if $\rho+\lambda\left(X_{0}+H_{0}\right)<\beta \omega(0,0)$, then by continuity we must have $\rho+\lambda\left(X_{0}+H_{0}\right)<\beta \omega(0, \lambda)$, when $\lambda$ is small. Next, recall from Lemma 4 that 
$t \rightarrow \omega(t, \lambda)$ is increasing. It follows then

$$
\begin{aligned}
\frac{\hat{\varphi}^{\prime}(t)}{\hat{\varphi}(t)} & =-\beta \frac{\hat{\phi}(t)-\hat{\psi}(t)}{\hat{\varphi}(t)}+\rho+\lambda H^{*}(t) \\
& =-\beta \omega(l, \lambda)+\rho+\lambda H^{*}(t)<-\beta \omega(0, \lambda)+\rho+\lambda\left(X_{0}+H_{0}\right)<0 .
\end{aligned}
$$

Hence the current shadow price of capital will be falling through time, and this in turn implies that consumption is rising through time.

The condition of Proposition 4 captures the idea that when the rate of time preference $\rho$ and the parameter $\lambda$ are both small, the effective rate of discount, which is the sum of $\rho$ and the risk premium associated with a possible catastrophe, $\lambda H^{*}(t)$, will be dominated by $-\beta \omega(t, \lambda)$ in time, with the ensuing consequence of a falling current shadow price of capital and a fortiori a rising level of consumption. The rising consumption reflects the fact that the threat of a catastrophe is not sufficient to deter the exploitation of the fossil fuel technology.

\subsection{Concluding remarks}

In the environmental economics literature, there are two hypotheses about the role of natural capital in the production process. One hypothesis assumes that physical capital (or man-made capital) can substitute for natural capital. The second, and perhaps more controversial, hypothesis states that natural capital complements the other factors, and cannot easily be substituted for by physical capital in the production of commodities. While it is reasonable to think that wind turbines and solar collectors can substitute for fossil fuels-as I assume in this model, it is difficult to imagine how physical capital can substitute for ecosystems in commodity production. Many ecologists believe that there is a critical level of natural capital embodied in ecosystems below which humanity will not survive. In the present paper, I have not 
modeled the impact of catastrophic climate change on the stock of natural capital. To keep the model more tractable at this stage, I have modeled the occurrence of catastrophes as a single random event, and have made the simplifying assumption that when a catastrophe occurs, consumption is permanently reduced to zero. In future research, these assumptions may be relaxed by allowing for the possibility of repeated catastrophes and by considering the role played by natural capital in more depth. 


\section{References}

[1] Ash, R. B., M. Gardner (1975): "Topics in Stochastic Processes," Probability and Methematical Statistics, vol. 27, Academic Press, New York.

[2] Berkovits, L. D. (1974): "Optimal Control Theory," Springer Verlag, BerlinHeidelberg-New York.

[3] Boyer, M., J. J. Laffont (1999): "Toward a Political Theory of the Emergence of Environmental Incentive Regulation," RAND Journal of Economics, 30:137-157.

[4] Brock, W. A., D. Starrett (1999): "Nonconvexities in Ecological Management Problems," Preprint, University of Wisconsin.

[5] Caputo, M.R.(2005): "Foundations of Dynamic Economic Analysis: Optimal Control Theory and Applications," Cambridge University Press, Cambridge

[6] Carpenter, S. R., D. Ludwig, and W. A. Brock (1999): "Management of Eutrophication for Lakes Subject to Potentially Irreversible Change," Ecological Applications, 9:751 771 .

[7] Clarke, H. R., W. J. Reed (1994): "Consumption/Pollution Trade-offs in an Environment Vulnerable to Pollution-related Catastrophic Collapse," Journal of Economic Dynamics and Control, 18:991 1010. 
[8] Cropper, M. L. (1976): "Regulating Activities with Catastrophic Environmental Effects," Journal of Environmental Economics and Management, 3:1 15.

[9] Dales, J. H. (1968): "Land, Water, and Ownership," Canadian Journal of Economics, 4:791 803 .

[10] Dasgupta, P. (2010): "The Concept of Natural Capital," Lecture notes, Inter Academy Panel Biodiversity Conference on Integrating Ecosystem Services into Biodiversity Management.

[11] - (2010), "Nature's Role in Sustainable Economic Development," Philosophical Transactions of the Royal Society B, 365:5 11.

[12] Dasgupta, P., K.-G. Mäler (2004): "The Economics of Non-convex Ecosystems: Introduction," Environmental and Resource Economics, 26:499 525.

[13] Dasgupta, P., G. Heal (1974): "The Optimal Depletion of Exhaustible Resources," Review of Economic Studies, 41:3 28.

[14] Dasgupta, P., P. Hammond, E. Maskin (1980): "On Imperfect Information and Optimal Pollution Control," Review of Economic Studies, 47: 85760.

[15] Dreyfus, S. E. (1965): "Dynamic Programming and the Calculus of Variations," Academic Press, New York.

[16] England, R. W. (2000): "Natural Capital and the Theory of Economic Growth," Ecological Economics, 34:425 431.

[17] Intergovernmental Panel on Climate Change (2001): "Climate Change 2001: Impacts, Adaptation, and Vulnerability,". Contribution of Working Group III to the Third Assessment Report of the IPCC. 

tribution of Working Group III to the Fourth Assessment Report of the Intergovernmental Panel on Climate Change [B. Metz, O.R. Davidson, P.R. Bosch, R. Dave, L.A. Meyer (eds.)], Cambridge University Press, Cambridge, United Kingdom and New York, NY, USA.

[19] IEA (2006): "World Energy Outlook 2006," International Energy Agency.

[20] Lucas, R. E. (1988): "On the Mechanics of Economic Development," Journal of Monetary Economics, 22:3 - 42.

[21] Mäler, K.-G., A. Xepapadeas, A. de Zeeuw (2000): "The Economics of Shallow Lakes," Preprint, The Beijer International Institute of Ecological Economics.

[22] - (2003): "The Economics of Shallow Lakes," Environmental and Resource Economics, 26:603 624.

[23] Ohm, P. (1981): "Deposit-Refund Systems: Theory and Applications to Environmental, Conservation, and Consumer Policy, Resources for the Future," John Hopkins University Press, Baltimore, MD.

[24] Pigou, A. C. (1960): "The Economics of Welfare," Fourth Edition, MacMillan, London

[25] Pindyck, R. S. (1984): "Uncertainty in the Theory of Renewable Resource Markets," Review of Economic studies, 51:289 303.

[26] Reed, W. J. (1984): "The Effect of the Risk of Fire on the Optimal Rotation of a Forest," Journal of Environmental Economics and Management, 11:180 190. (1989): "Optimal Investment in the Protection of a Vulnerable Biological Resource," Natural Resource Modeling, 3:463 480. 
[28] Segerson, K. (1995): "Liability and Penalty Structures in Policy Design," in D. W. Bromley (ed.), Handbook of Environmental Economics, Basil Blackwell, Cambridge, MA.

[29] (1996): "Issues in the Choice of Environmental Policy Instruments," in J. B. Braden, H. Folmer and T. S. Ulen (eds.), Environmental Policy with Political and Economic Integration, Edward Elgar Publishing Inc., Cheltenham, UK.

[30] Skiba, A. K. (1978): "Optimal Growth with a Convex-Concave Production Function," Econometrica, 46:527 540.

[31] Stavins, R. (2000): "Experience with Market-Based Environmental Policy Instruments," in K. Mäler and J. Vincent (eds.), The Handbook of Environmental Economics, vol. 1, North-Holland/Elsevier Science, Amsterdam.

[32] Stocker, T. F., A. Schmittner (1997): "Influence of $\mathrm{CO}_{2}$ Emissions Rates on the Stability of the Thermohaline Circulation," Nature, 388:862 865.

[33] Tsur, Y., A. Zemel (1995): "Uncertainty and Irreversibility in Groundwater Management," Journal of Environmental Economics and Management, 29:149 161.

[34] … (1996): "Accounting for Global Warming Risks: Resource Management under Event Uncertainty, Journal of Economic Dynamics and Control, 20:1289 1305.

[35] - (1998): "Pollution Control in an Uncertain Environment," Journal of Economic Dynamics and Control, 22: 967975. 
[36] Wagener, F. O. (2003): "Skiba Points and Heteroclinic Bifurcations, with Applications to the Shallow Lake System," Journal of Economic Dynamics and Control, 27:1533 1561 . 


\section{Appendix 3.A The derivation of the HJB equation and the adjoint equations}

\section{A.1 The Hamilton-Jacobi-Bellman equation}

Let $t \rightarrow\left(K_{1}, x, c\right), t \geq \tau$, be an admissible control policy from the initial condition $(\xi, \eta, \zeta, \tau)$. If we carry out this control policy during an infinitesimal time interval $[\tau, \tau+\Delta t)$, and continue optimally afterward from the new initial condition $(X(\tau+$ $\Delta t), K(\tau+\Delta t), H(\tau+\Delta t))$, then the expected discounted welfare obtained is given by

$$
\begin{aligned}
e^{-\rho \tau} u(c(\tau)) \Delta t+(1-\lambda H(\tau) \Delta t+o(\Delta t)) \times & \\
& v(X(\tau+\Delta t), K(\tau+\Delta t) . H(\tau+\Delta t), \tau+\Delta t)+o(\Delta t),
\end{aligned}
$$

where $o(\Delta t)$ is a term in $\Delta t$, such that $\lim _{\Delta t \rightarrow 0} \frac{o(\Delta t)}{\Delta t}=0$.

Note that in (3.A.1), the term $e^{-\rho \tau} u(c(\tau)) \Delta t$ represents the discounted welfare obtained during the infinitesimal time interval $[\tau, \tau+\Delta t)$, and the expression

$$
(1-\lambda H(\tau) \Delta t+o(\Delta t)) v(X(\tau+\Delta t), K(\tau+\Delta t), H(\tau+\Delta t), \tau+\Delta t)
$$

represents the expected discounted welfare under the event that no catastrophe occurs during the infinitesimal time interval $[\tau, \tau+\Delta t)$. This event has probability $(1-\lambda H(\tau) \Delta t+o(\Delta t))$. The event that a catastrophe occurs during the infinitesimal time interval $[\tau, \tau+\Delta t)$ has probability $\lambda H(\tau) \Delta t+o(\Delta t)$, and this event yields a consumption level of 0 forever after that.

Clearly, (3.A.1) cannot exceed $v(\xi, \eta, \zeta, \tau)$, the expected discounted social welfare obtained by carrying out the optimal policy immediately from the initial condition 
$(\xi, \eta, \zeta, \tau)$. That is

$$
\begin{aligned}
& e^{-\rho \tau} u(c(\tau)) \Delta t+(1-\lambda H(\tau) \Delta t+o(\Delta l)) \times \\
& \quad v(X(\tau+\Delta t), K(\tau+\Delta t), H(\tau+\Delta t), \tau+\Delta t)+o(\Delta t) \leq v(\xi, \eta, \zeta, \tau)
\end{aligned}
$$

with equality holding if the control policy $t \rightarrow\left(K_{1}, x, c\right), t \geq \tau$, is optimal from the initial condition $(\xi, \eta, \zeta, \tau)$.

Let us rewrite (3.A.2) as follows:

$$
\begin{aligned}
& e^{-\rho \tau} u(c(\tau)) \Delta t+v(X(\tau+\Delta t), K(\tau+\Delta t), H(\tau+\Delta t), \tau+\Delta t)+o(\Delta t)-v(\xi, \eta, \zeta, \tau) \\
& -(\lambda H(\tau) \Delta t+o(\Delta t)) v(X(\tau+\Delta t), K(\tau+\Delta t), H(\tau+\Delta t), \tau+\Delta t)+o(\Delta t) \leq 0
\end{aligned}
$$

with equality holding if the control policy $t \rightarrow\left(K_{1}, x, c\right), t \geq \tau$, is optimal from the initial condition $(\xi, \eta: \zeta, \tau)$.

Dividing (3.A.3) by $\Delta t$, and then letting $\Delta t \rightarrow 0$, we obtain

$$
\begin{aligned}
& e^{-\rho \tau} u(c(\tau))+D_{1} v(\xi, \eta, \zeta, \tau)\left(\frac{d X}{d t}\right)_{t=\tau}+D_{2} v(\xi, \eta, \zeta, \tau)\left(\frac{d K}{d t}\right)_{t=\tau} \\
& +D_{3} v(\xi, \eta, \zeta, \tau)\left(\frac{d H}{d t}\right)_{t=\tau}+D_{4} v(\xi, \eta, \zeta, \tau)-\lambda \zeta v(\xi, \eta, \zeta, \tau) \\
= & e^{-\rho \tau} u(c(\tau))-D_{1} v(X(\tau), K(\tau), H(\tau), \tau) x(\tau) \\
& +D_{2} v(X(\tau), K(\tau), H(\tau), \tau)\left(A K_{1}^{\alpha}(\tau)\left(x(\tau)+\beta\left(K(\tau)-K_{1}(\tau)\right)\right)^{1-\alpha}-c(\tau)\right) \\
& +D_{3} v(X(\tau), K(\tau), H(\tau), \tau)(x(\tau)-\delta H(\tau))-\lambda H(\tau) v(X(\tau), K(\tau), H(\tau), \tau) \\
& +D_{4} v(X(\tau), K(\tau), H(\tau), \tau) \leq 0,
\end{aligned}
$$

with equality holding if the control policy $\left(K_{1}(\tau), x(\tau), c(\tau)\right)$ is optimal at the initial condition $(\xi, \eta, \zeta, \tau)$.

We can express the results just obtained under the following more compact form, known as the Hamilton-Jacobi-Bellman equation of dynamic programming: 


$$
\begin{array}{r}
0=\max _{\left(K_{1}, x, c\right)}\left(e^{-\rho \tau} u(c)-D_{1} v(\xi, \eta, \zeta, \tau) x+D_{2} v(\xi, \eta, \zeta, \tau)\left(A K_{1}^{\alpha}\left(x+\beta\left(\eta-K_{1}\right)\right)^{1-\alpha}-c\right)\right. \\
\left.+D_{3} v(\xi, \eta, \zeta, \tau)(x-\delta \zeta)-\lambda \zeta v(\xi, \eta, \zeta, \tau)+D_{4} v(\xi, \eta, \zeta, \tau)\right), \quad \text { (3.A.5) }
\end{array}
$$

and the values of $K_{1}, x$, and $c$ that solves the maximization problem in (3.A.5) are, respectively, the optimal capital allocated to the consumption good sector, the optimal oil extraction rate, and the optimal consumption - all at the initial condition $(\xi, \eta, \zeta ; \tau)$

\section{A.2 The motion of the shadow prices}

Let us momentarily fix $(\xi, \eta, \zeta, \tau)$. Next, let $K_{1}(\xi, \eta, \zeta, \tau), x(\xi, \eta, \zeta, \tau)$, and $c(\xi, \eta, \zeta, \tau)$ be the values of $K_{1}, x$, and $c$, respectively, that solve the maximization problem in the Hamilton-Jacobi-Bellman equation (3.A.5).

Now suppose that the economy begins at time $t$, with $X$ as the remaining stock of oil, $K$ as the stock of capital, and $H$ as the stock of greenhouse gases in the atmosphere. Define

$$
\begin{array}{r}
\mathbb{M}(X, K, I I, t)=e^{-\rho t} u(c(\xi, \eta, \zeta, \tau))-D_{1} v(X, K, H, t) x(\xi, \eta, \zeta, \tau) \\
+D_{2} v(X, K, I I, t) A\left(K_{1}(\xi, \eta, \zeta, \tau)\right)^{\alpha}\left(x(\xi, \eta, \zeta, \tau)+\beta\left(K-K_{1}(\xi, \eta, \zeta, \tau)\right)^{1-\alpha}\right. \\
-c(\xi, \eta, \zeta, \tau))+D_{3} v(X, K, H, t)(x(\xi, \eta, \zeta, \tau)-\delta H) \\
-\lambda H v(X, K, H, t)+D_{4} v(X, K, H, t)
\end{array}
$$

Because $K_{1}(\xi, \eta, \zeta, \tau), x(\xi, \eta, \zeta, \tau)$, and $c(\xi, \eta, \zeta, \tau)$ are not necessarily optimal at $(X, K, H, t)$, we must have $\mathbb{M}(X, K, H, t) \leq 0$ for all $(X, K, H, t)$, according to the Hamilton-Jacobi-Bellman equation (3.A.j). Thus, the map $\mathbb{M}:(X, K, H, t) \rightarrow$ $\mathbb{M}(X, K, H, t)$, which are always less than or equal to 0 , achieves a global maximum 
equal to 0 at $(X, K, H, t)=(\xi, \eta, \zeta, \tau)$, and the following first-order conditions hold:

$$
\begin{aligned}
& D_{1} \mathbb{M}(\xi, \eta, \zeta, \tau)=-D_{11} v(\xi, \eta, \zeta, \tau) x(\xi, \eta, \zeta, \tau) \\
& +D_{21} v(\xi, \eta, \zeta, \tau) A\left(K_{1}(\xi, \eta, \zeta, \tau)\right)^{\alpha}\left(x(\xi, \eta, \zeta, \tau)+\beta\left(K-K_{1}(\xi, \eta, \zeta, \tau)\right)^{1-\alpha}\right. \\
& -c(\xi, \eta, \zeta, \tau))+D_{31} v(\xi, \eta, \zeta, \tau)(x(\xi, \eta, \zeta, \tau)-\delta \zeta) \\
& \quad-\lambda H D_{1} v(\xi, \eta, \zeta, \tau)+D_{41} v(\xi, \eta, \zeta, \tau)=0, \quad \text { 3.A.7) } \\
& \begin{array}{r}
D_{2} \mathbb{M}(\xi, \eta, \zeta, \tau)=-D_{12} v(\xi, \eta, \zeta, \tau) x(\xi, \eta, \zeta, \tau) \\
+D_{22} v(\xi, \eta, \zeta, \tau) A\left(K_{1}(\xi, \eta, \zeta, \tau)\right)^{\alpha}\left(x(\xi, \eta, \zeta, \tau)+\beta\left(K-K_{1}(\xi, \eta, \zeta, \tau)\right)^{1-\alpha}\right. \\
\quad-c(\xi, \eta, \zeta, \tau))+D_{2} v(\xi, \eta, \zeta, \tau) A\left(K_{1}(\xi, \eta, \zeta, \tau)\right)^{\alpha}(x(\xi, \eta, \zeta, \tau) \\
\left.\quad+\beta\left(K-K_{1}(\xi, \eta, \zeta, \tau)\right)^{-\alpha} \beta(1-\alpha)\right) \\
+D_{32} v(\xi, \eta, \zeta, \tau)(x(\xi, \eta, \zeta, \tau)-\delta \zeta)-\lambda H D_{2} v(\xi, \eta, \zeta, \tau)+D_{42} v(\xi, \eta, \zeta, \tau)=0
\end{array}
\end{aligned}
$$

and

$$
\begin{aligned}
& D_{3} \mathbb{M}(\xi, \eta, \zeta, \tau)=-D_{13} v(\xi, \eta, \zeta, \tau) x(\xi, \eta, \zeta, \tau) \\
&+D_{23} v(\xi, \eta, \zeta, \tau) A\left(K_{1}(\xi, \eta, \zeta, \tau)\right)^{\alpha}\left(x(\xi, \eta, \zeta, \tau)+\beta\left(K-K_{1}(\xi, \eta, \zeta, \tau)\right)^{1-\alpha}\right. \\
&-c(\xi, \eta, \zeta, \tau))+D_{33} v(\xi, \eta, \zeta, \tau)(x(\xi, \eta, \zeta, \tau)-\delta \zeta)-\delta D_{3} v(\xi, \eta, \zeta, \tau) \\
& \quad-\lambda v(X, K, H, t)-\lambda \zeta D_{3} v(\xi, \eta, \zeta, \tau)+D_{43} v(\xi, \eta, \zeta, \tau)=0 .
\end{aligned}
$$

Let $\left(K_{1}^{*}, x^{*}, c^{*}\right), t \geq 0$, be the solution of the central planner's problem as stated by $(3.2 .22)(3.2 .25)$. Under the optimal control policy, the remaining stock of oil evolves according to the following differential equation:

$$
\begin{aligned}
\frac{d X^{*}}{d t} & =-x^{*} \\
X^{*}(0) & =X_{0}>0 \text { is given, }
\end{aligned}
$$

and the stock of capital evolves according to the following differential equation: 


$$
\begin{gathered}
\frac{d K^{*}}{d t}=\Lambda\left(K_{1}^{*}\right)^{\alpha}\left(x^{*}+\beta\left(K^{*}-K_{1}^{*}\right)\right)^{1-\alpha}-c^{*}, \\
K^{*}(0)=K_{0}>0 \text { is given. }
\end{gathered}
$$

As for the stock of greenhouse gases in the atmosphere, its motion is governed by the following differential equation:

$$
\begin{aligned}
\frac{d H^{*}}{d t} & =x^{*}-\delta H^{*} \\
H^{*}(0) & =H_{0} \text { is given. }
\end{aligned}
$$

Let

$$
\begin{aligned}
& \phi=D_{1} v\left(X^{*}, K^{*}, H^{*}, t\right), \\
& \varphi=D_{2} v\left(X^{*}, K^{*}, H^{*}, t\right),
\end{aligned}
$$

and

$$
\psi=D_{3} v\left(X^{*}, K^{*}, H^{*}, t\right)
$$

denote, respectively, the shadow price of oil, the shadow price of capital, and the shadow price of greenhouse gases in the atmosphere - all at time $t$ along the optimal trajectory.

\section{A.2.1 The motion of the shadow price of oil}

We have

$$
\begin{aligned}
\frac{d \phi}{d t} & =D_{11} v\left(X^{*}(l), K^{*}(l), H^{*}(t), \iota\right) \frac{(d X)^{*}}{d t}+D_{12} v\left(X^{*}(t), K^{*}(t), H^{*}(l), l\right) \frac{(d K)^{*}}{d t} \\
& +D_{13} v\left(X^{*}(t), K^{*}(t), H^{*}(t), t\right) \frac{(d H)^{*}}{d t}+D_{14} v\left(X^{*}(t), K^{*}(t), H^{*}(t), t\right) .
\end{aligned}
$$


Applying (3.A.7) at $(\xi, \eta, \zeta, \tau)=\left(X^{*}, K^{*}, H^{*}, t\right)$, we obtain

$$
\begin{aligned}
& -D_{11} v\left(X^{*}, K^{*}, H^{*}, l\right) x^{*}+D_{21} v\left(X^{*}, K^{*}, H^{*}, l\right) A\left(K_{1}^{*}\right)^{\alpha}\left(x^{*}+\beta\left(K^{*}-K_{1}^{*}\right)^{1-\alpha}-c^{*}\right) \\
& +D_{31} v\left(X^{*}, K^{*}, H^{*}, l\right)\left(x^{*}-\delta H^{*}\right)-\lambda H^{*} D_{1} v\left(X^{*}, K^{*}, H^{*}, l\right)+D_{41} v\left(X^{*}, K^{*}, H^{*}, l\right) \\
& =D_{11} v\left(X^{*}, K^{*}, H^{*}, t\right) \frac{d X^{*}}{d t}+D_{12}\left(X^{*}, K^{*}, H^{*}, t\right) \frac{d K^{*}}{d t}+D_{13}\left(X^{*}, K^{*}, H^{*}, t\right) \frac{d H^{*}}{d t} \\
& +D_{14} v\left(X^{*}, K^{*}, H^{*}, t\right)-\lambda H^{*} D_{1} v\left(X^{*}, K^{*}, H^{*}, t\right) \\
& =\frac{d \phi}{d t}-\lambda H^{*} D_{1} v\left(X^{*}, K^{*}, H^{*}, t\right)=0 .
\end{aligned}
$$

Note that the first equality in (3.A.17) has been obtained with the help of Young's theorem $\left(D_{i j} v(X, K, H, t)=D_{i j} v(X, K, H, t)\right)$, and the second equality with the help of (3.A.16).

It now follows from (3.A.15) that

$$
\begin{aligned}
\frac{d \phi}{d t} & =\lambda H^{*} D_{1} v\left(X^{*}, K^{*} \cdot H^{*}, t\right) \\
& =\lambda H^{*} \phi
\end{aligned}
$$

Let us define the Hamiltonian as

$$
\begin{array}{r}
\mathscr{H}\left(X, K, H, K_{1}, x, c, \phi, \varphi, \psi, t\right)=e^{-\rho t} u(c)-\phi x+\varphi A K_{1}^{\alpha}\left(x+\beta\left(\eta-K_{1}\right)^{1-\alpha}-c\right) \\
+\psi(x-\delta \zeta)-\lambda H v(X, K, H, t)+D_{4} v(X, K, H, t) .
\end{array}
$$

Then (3.A.16), the differential equation governing the motion of the shadow price of oil, can be represented under the following familiar form:

$$
\begin{aligned}
\frac{d \phi}{d t} & =-D_{1} \mathscr{H}\left(X^{*}, K^{*}, H^{*}, K_{1}^{*}, x^{*}, c^{*}, \phi, \varphi, \psi, t\right) \\
& =\lambda H^{*} \phi
\end{aligned}
$$




\section{A.2.2 The motion of the shadow price of capital}

We have

$$
\begin{aligned}
\frac{d \varphi}{d t}=D_{21} v\left(X^{*}, K^{*},\right. & \left.H^{*}, t\right) \frac{d X^{*}}{d t}+D_{22} v\left(X^{*}, K^{*}, H^{*}, t\right) \frac{d K^{*}}{d t} \\
& +D_{23} v\left(X^{*}, K^{*}, H^{*}, t\right) \frac{d I^{*}}{d t}+D_{24} v\left(X^{*}, K^{*}, H^{*}, t\right) .
\end{aligned}
$$

Applying (3.A.8) at $(\xi, \eta, \zeta, \tau)=\left(X^{*}, K^{*}, H^{*}, t\right)$, we obtain

$$
\begin{aligned}
& -D_{12} v\left(X^{*}, K^{*}, H^{*}, t\right) x^{*}+D_{22} v\left(X^{*}, K^{*}, H^{*}, t\right) A\left(K_{1}^{*}\right)^{\alpha}\left(x^{*}+\beta\left(K^{*}-K_{1}^{*}\right)^{1-\alpha}-c^{*}\right) \\
& +D_{2} v\left(X^{*}, K^{*}, H^{*}, t\right) A\left(K_{1}^{*}\right)^{\alpha}\left(x^{*}+\beta\left(K^{*}-K_{1}^{*}\right)^{-\alpha} \beta(1-\alpha)\right) \\
& +D_{32} v\left(X^{*}, K^{*}, H^{*}, t\right)\left(x^{*}-\delta H^{*}\right)-\lambda H^{*} D_{2} v\left(X^{*} \cdot K^{*}, H^{*}, t\right)+D_{42} v\left(X^{*}, K^{*}, H^{*}, t\right) \\
& =D_{21} v\left(X^{*}, K^{*}, H^{*}, t\right) \frac{d X^{*}}{d t}+D_{22}\left(X^{*}, K^{*}, H^{*}, t\right) \frac{d K^{*}}{d t} \\
& \quad+D_{2} v\left(X^{*}, K^{*}, H^{*}, t\right) A\left(K_{1}^{*}\right)^{\alpha}\left(x^{*}+\beta\left(K^{*}-K_{1}^{*}\right)^{-\alpha} \beta(1-\alpha)\right) \\
& \quad+D_{23}\left[X^{*}, K^{*}, H^{*}, t\right) \frac{d H^{*}}{d t}+D_{24} v\left(X^{*}, K^{*}, H^{*}, t\right)-\lambda H^{*} D_{2} v\left(X^{*}, K^{*}, H^{*}, t\right) \\
& =\frac{d \varphi}{d t}+D_{2} v\left(X^{*}, K^{*}, H^{*}, t\right) A\left(K_{1}^{*}\right)^{\alpha}\left(x^{*}+b\left(K^{*}-K_{1}^{*}\right)-\alpha b(1-\alpha)\right) \\
& \quad-\lambda H^{*} D_{2} v\left(X^{*}, K^{*}, H^{*}, t\right)=0 .
\end{aligned}
$$

Note that the first equality in (3.A.22) has been obtained with the help of Young's theorem $D_{i j} v(X, K, H, t)=D_{j i} v(X, K, H, t)$, and the second equality with the help of (3.A.21). It now follows from (3.A.22) that

$$
\begin{aligned}
\frac{d \varphi}{d t}= & -D_{2} v\left(X^{*}, K^{*}, H^{*}, t\right) A\left(K_{1}^{*}\right)^{\alpha}\left(x^{*}+\beta\left(K^{*}-K_{1}^{*}\right)^{-\alpha} b(1-\alpha)\right) \\
& +\lambda H^{*} D_{2} v\left(X^{*}, K^{*}, H^{*}, t\right) \\
= & -\varphi\left(A\left(K_{1}^{*}\right)^{\alpha}\left(x^{*}+\beta\left(K^{*}-K_{1}^{*}\right)^{-\alpha} \beta(1-\alpha)\right)+\lambda H^{*} \varphi\right. \\
= & -D_{2} \mathscr{H}\left(X^{*}, K^{*}, H^{*}, K_{1}^{*}, x^{*}, c^{*}, \phi, \varphi, \psi, t\right) .
\end{aligned}
$$




\section{A.2.3 The motion of the shadow price of greenhouse gases in the at-} mosphere

We have

$$
\begin{aligned}
\frac{d \psi}{d t}=D_{31} v\left(X^{*}, K^{*},\right. & \left.H^{*}, t\right) \frac{d X^{*}}{d t}+D_{32} v\left(X^{*}, K^{*}, H^{*}, t\right) \frac{d K^{*}}{d t} \\
& +D_{33} v\left(X^{*}, K^{*}, H^{*}, t\right) \frac{d H^{*}}{d t}+D_{34} v\left(X^{*}, K^{*}, H^{*}, t\right) .
\end{aligned}
$$

Applying (3.A.9) at $(\xi, \eta, \zeta, \tau)=\left(X^{*}, K^{*}, H^{*}, t\right)$, we obtain

$$
\begin{aligned}
& -D_{13} v\left(X^{*}, K^{*}, H^{*}, t\right) x^{*}+D_{23} v\left(X^{*}, K^{*}, H^{*}, t\right) A\left(K_{1}^{*}\right)^{\alpha}\left(x^{*}+\beta\left(K^{*}-K_{1}^{*}\right)^{1-\alpha}-c^{*}\right) \\
& +D_{33} v\left(X^{*}, K^{*}, H^{*}, t\right)\left(x^{*}-\delta H^{*}\right)-\delta D_{3} v\left(X^{*}, K^{*}, H^{*}, t\right)-\lambda v\left(X^{*}, K^{*}, H^{*}, t\right) \\
& -\lambda H^{*} D_{3} v\left(X^{*}, K^{*}, H^{*}, t\right)+D_{43} v\left(X^{*}, K^{*}, H^{*}, t\right) \\
& =D_{31} v\left(X^{*}, K^{*}, H^{*}, t\right) \frac{d X^{*}}{d t}+D_{32}\left[X^{*}, K^{*}, H^{*}, t\right) \frac{d K^{*}}{d t}+D_{33}\left[X^{*}, K^{*}, H^{*}, t\right) \frac{d H^{*}}{d t} \\
& -\delta D_{3} v\left(X^{*}, K^{*}, H^{*}, t\right)+D_{34} v\left(X^{*}, K^{*}, H^{*}, t\right)-\lambda v\left(X^{*}, K^{*}, H^{*}, t\right) \\
& -\lambda H^{*} D_{3} v\left(X^{*}, K^{*}, H^{*}, t\right) \\
& =\frac{d \psi}{d t}-\delta D_{3} v\left(X^{*}, K^{*}, H^{*}, t\right)-\lambda v\left(X^{*}, K^{*}, H^{*}, t\right)-\lambda H^{*} D_{3} v\left(X^{*}, K^{*}, H^{*}, t\right)=0 .
\end{aligned}
$$

Note that the first equality in (3.A.25) has been obtained with the help of Young's theorem, $D_{i j} v(X, K, H, t)=D_{j i} v(X, K, H, t)$, and the second equality with the help of (3.A.24).

It now follows from (3.A.24) that

$$
\begin{aligned}
\frac{d \psi}{d t} & =\delta D_{3} v\left(X^{*}, K^{*}, H^{*}, t\right)+\lambda D_{3} v\left(X^{*}, K^{*}, H^{*}, t\right)+\lambda H^{*} D_{3} v\left(X^{*}, K^{*}, H^{*}, t\right) \\
& =\left(\delta+\lambda I^{*}\right) \psi+\lambda v\left(X^{*}, K^{*}, I^{*}, t\right) \\
& =-D_{3} \mathbb{M}\left(X^{*}, K^{*}, I^{*}, K_{1}^{*}, x^{*}, c^{*}, \phi, \varphi, \psi, t\right)
\end{aligned}
$$

